\title{
Mechanisms of Alphavirus Midgut Dissemination in the Mosquito, Aedes aegypti
}

A Dissertation Presented to the Faculty of the Graduate School at the University of Missouri-Columbia

In Partial Fulfillment of the Requirements for the Degree

Doctor of Philosophy

By

Asher M. Kantor

Dr. Alexander W.E. Franz, Dissertation Supervisor

December 2018 
The undersigned, appointed by the dean of the Graduate School, have examined the dissertation entitled

Mechanisms of Alphavirus Midgut Dissemination in the Mosquito, Aedes aegypti

Presented by Asher M. Kantor,

a candidate for the degree of doctor of philosophy, and hereby certify that, in their opinion, is worthy of acceptance.

Dr. Alexander W.E. Franz

Dr. Brenda T. Beerntsen

Dr. Deborah Anderson

Dr. Roger W. Stich

Dr. Marc Johnson 


\section{DEDICATION}

I would like to dedicate this dissertation to my Mom and Dad who have always supported and enabled me to follow my passions. I would also like to thank my Grandparents Bernard and Eleanor Lutzk, and David and Clarice Kantor, who taught me from a young age to always be inquisitive. I am also grateful to my brother who has always been a worthy role model to look up to, and has always challenged and accompanied me as we explore. 


\section{ACKNOWLEDGEMENTS}

First and foremost, I would like to thank my advisor, Dr. Alexander Franz. I am grateful for his training, guidance, and knowledge. Beginning a career in science has challenged me but I am thankful to have had Dr. Franz as a mentor to guide me along the way. Working under Dr. Franz has provided me with a skill set that I can apply as I continue my career.

Additionally, I would like to acknowledge each of the members of my Dissertation Committee, Dr. Deborah Anderson, Dr. Bill Stich, Dr. Brenda Beerntsen, and Dr. Marc Johnson. Each of them has provided me with a great deal of advice and encouragement. Their feedback has guided me to success in these scientific efforts. In addition, my growth as a scientist has also been cultivated by many collaborators and colleagues including Dr. Nicole Held, Dr. Vel Balaraman, Dr. Shengzhang Dong, Dr. Xiudao Yu, Dr. William Reid, and Dr. Yingjun Cui, and Michelle Gregory. I would like to give a special thank you to Jingyi Lin who helped maintain multiple mosquito colonies and cell lines, and made it possible to complete experiments in a timely manner. I would also like to acknowledge Dr. Tommi White, DeAna Grant, and the rest of the Mizzou Electron Microscopy Core. Their contributions added vastly to our work and made the research in Chapter IV possible.

I would also like to thank my fellow graduate students in Connaway Hall. It was great to be around a motivated group of students each and every day. 
Everyone was always willing to share ideas, reagents, and a positive mental attitude. Finally, I express my gratitude to all of the educators I have come across in my life for the influence they had on me. Their hard work does not go unnoticed. 


\section{TABLE OF CONTENTS}

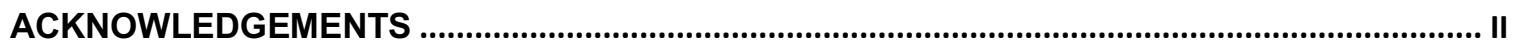

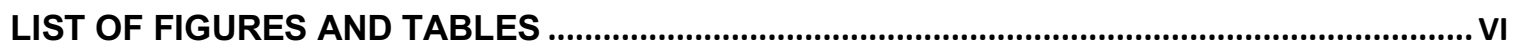

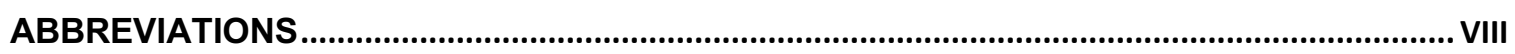

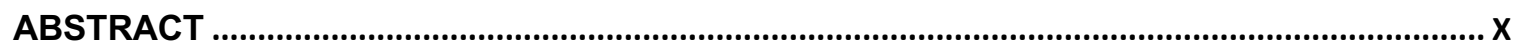

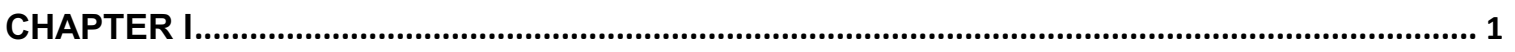

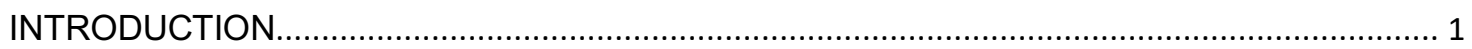

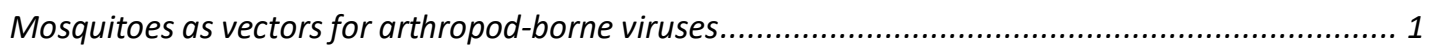

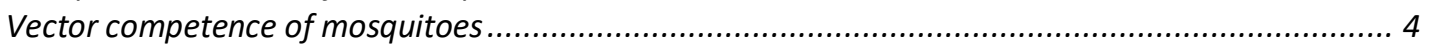

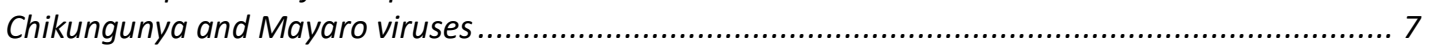

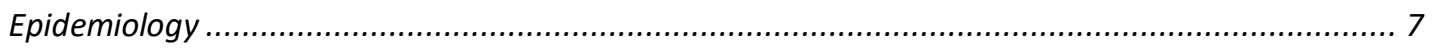

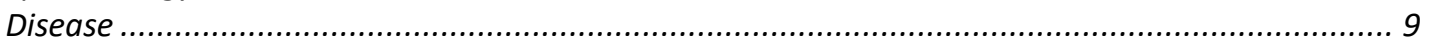

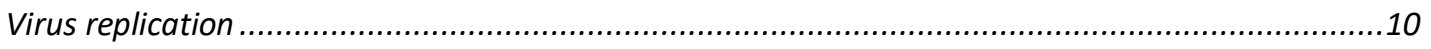

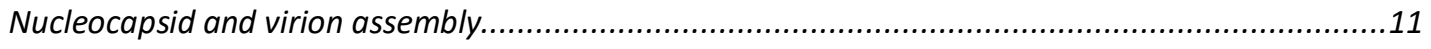

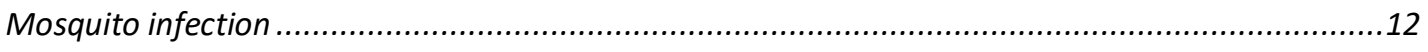

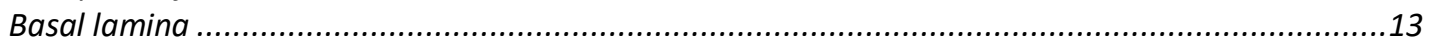

Potential role for matrix metalloproteinases in BL remodeling..................................................16

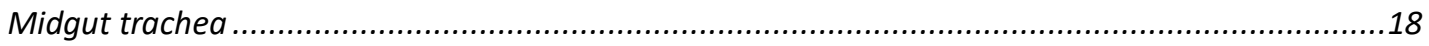

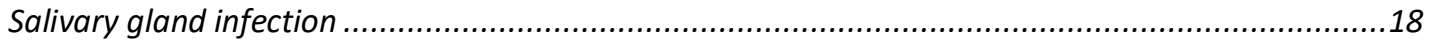

Immune responses to alphaviruses in mosquitoes ....................................................................19

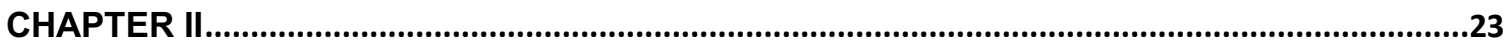

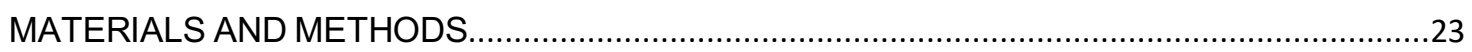

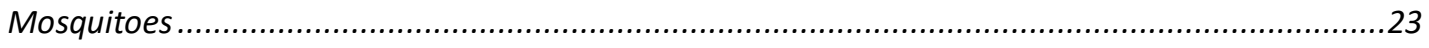

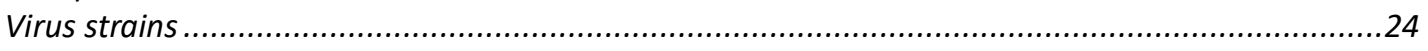

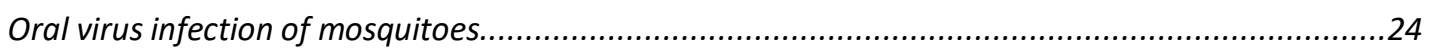

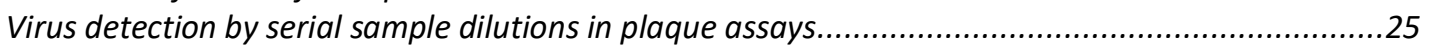

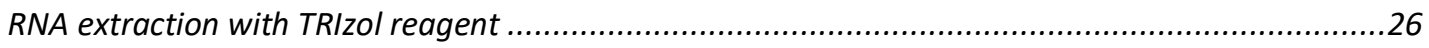

Database searches and phylogenetic analysis of matrix-metalloproteinase (MMPs) .......................27

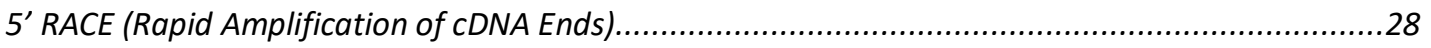

CDNA synthesis from mosquito MRNA and SYBR green-based quantitative (q)PCR........................28

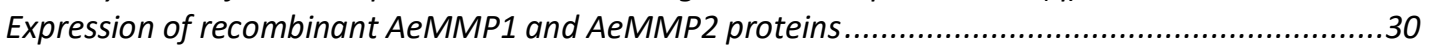

Generation of AeMMP1 and AeMMP2-specific antibodies and their use in Western blot analysis....30

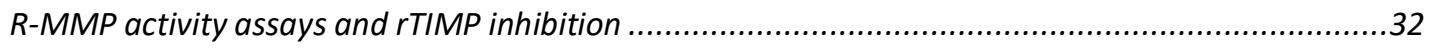

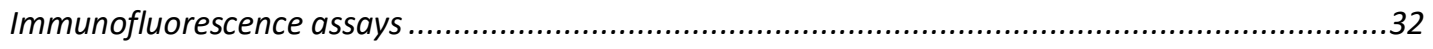

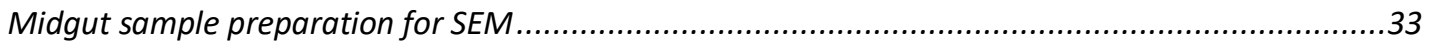

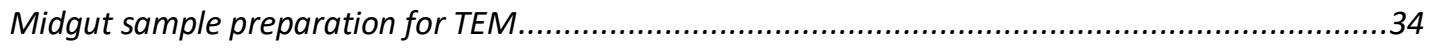

Analyzing chikungunya (CHIK) virion density in midgut epithelial cells and measuring midgut $B L$

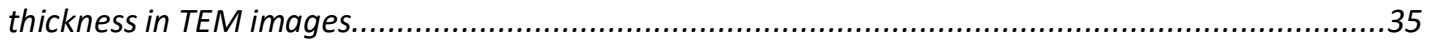

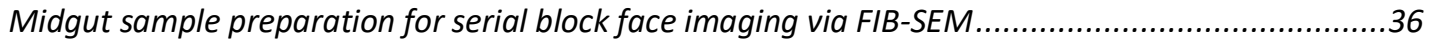

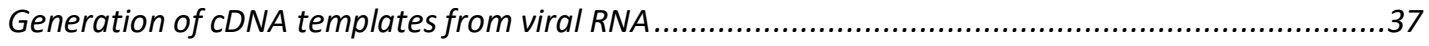

Taqman qPCR assays for the virus-specific detection of plus- and minus-strand RNAs.....................38

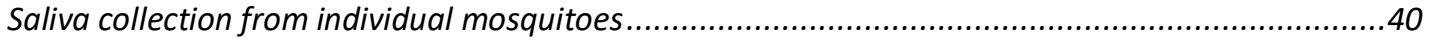

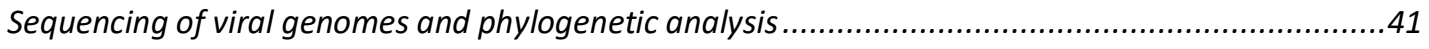

qRT-PCR for virus-specific detection in samples containing mixed infections ...................................42

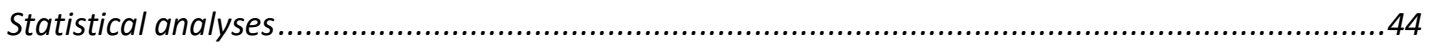

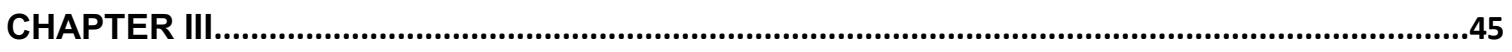




\section{IDENTIFICATION AND INITIAL CHARACTERIZATION OF MATRIX METALLOPROTEINASES IN THE YELLOW

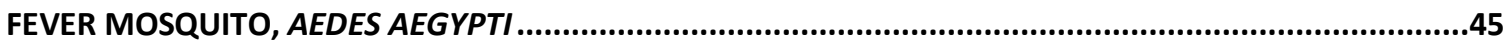

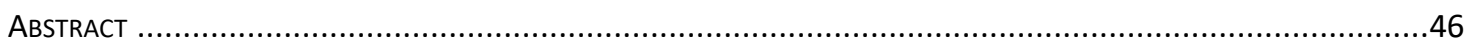

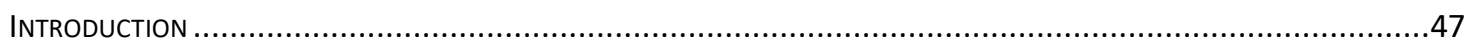

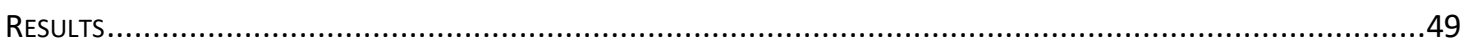

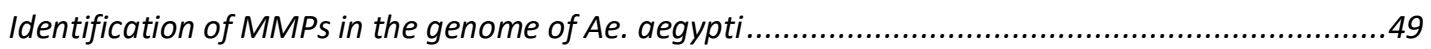

Similarity of AeMMPs with MMPs of humans and other arthropods............................................53

Expression profiles of AeMMP genes during different life stages of Ae. aegypti and in females that

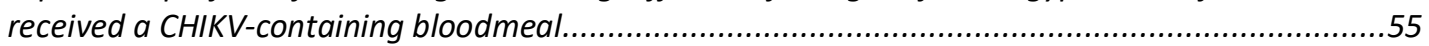

Detection of AeMMP1 and AeMMP2 proteins in midguts of Ae. aegypti.....................................6

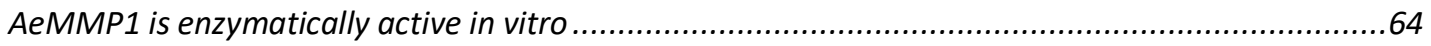

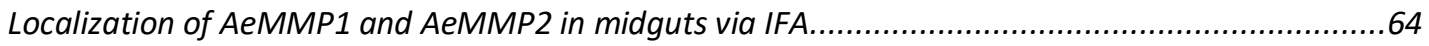

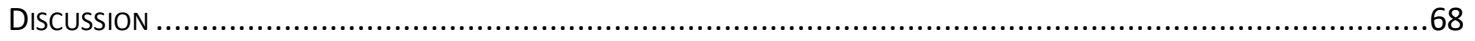

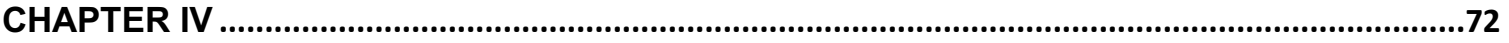

ULTRASTRUCTURAL ANALYSIS OF CHIKUNGUNYA VIRUS DISSEMINATION FROM THE MIDGUT OF THE YELLOW FEVER MOSQUITO, AEDES AEGYPTI ..........................................................................................72

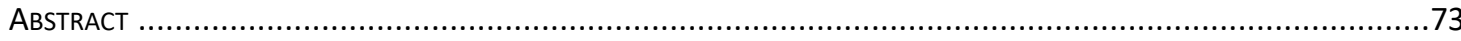

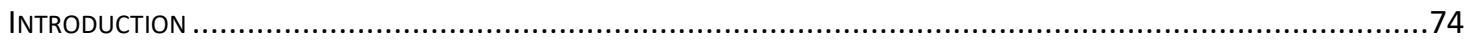

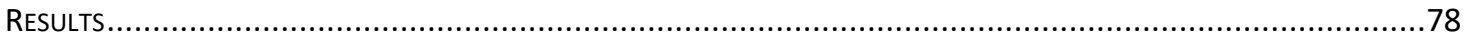

Bloodmeal ingestion causes temporal midgut tissue expansion and damage to the $B L$....................78

CHIKV disseminates from the midgut within a narrow time window during bloodmeal digestion .....83

Repeated bloodmeals enhance virion dissemination from the midgut epithelium ...........................95

Quantification of alphavirus RNA in infected midguts and carcasses over time indicates that virus

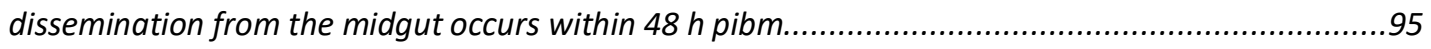

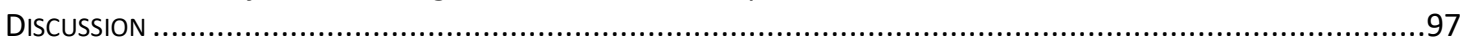

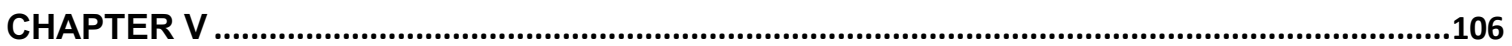

INFECTION PATTERN OF MAYARO VIRUS IN AEDES AEGYPTI AND TRANSMISSION POTENTIAL OF THE VIRUS IN MIXED INFECTIONS WITH CHIKUNGUNYA VIRUS ...............................................................106

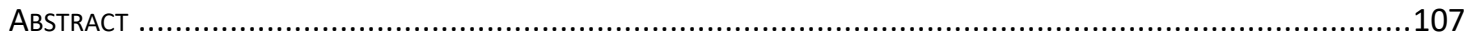

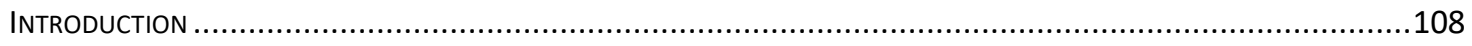

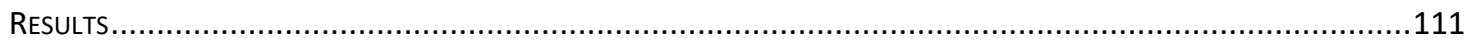

Phylogenetic characterization of MAYV strains IQT 4235 and TRVL 4675...................................111

Midgut and carcass infection of HWE and ORL mosquitoes with the IQT and TRVL strains of MAYV

MAYV head tissue infections and prevalence of the virus in saliva of HWE and ORL mosquitoes.....117

MAYV TRVL infection pattern in local Ae. albopictus .................................................................119

The effects of mixed infections between CHIKV 37997 and MAYV IQT on the transmission potential of

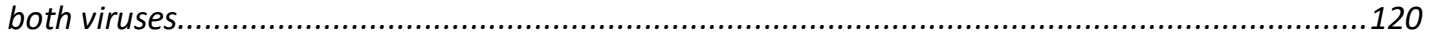

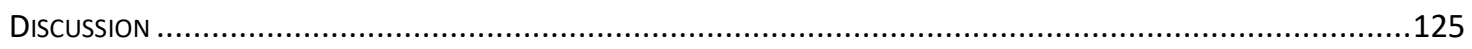

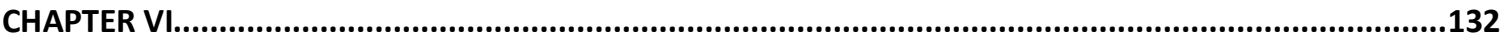

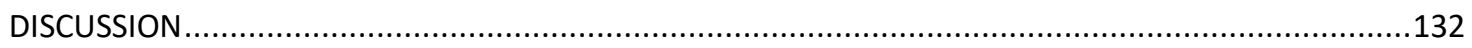

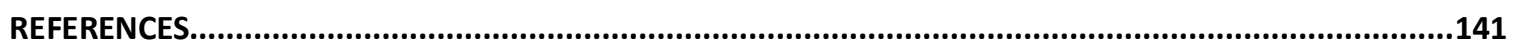

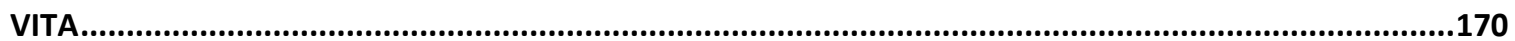




\section{LIST OF FIGURES AND TABLES}

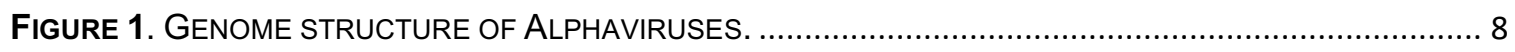

FIGURE 2. PATHWAY OF ARBOVIRUS DISSEMINATION IN AE. AEGYPTI. ..............................................14

FigURE 3. ULTRASTRUCTURAL VIEWS OF THE POSTERIOR MIDGUT TISSUE OF AE. AEGYPTI. ...................15

FIgURE 4. CHARACTERIZATION OF THE FUNCTIONAL DOMAINS OF AE. AEGYPTI MATRIX

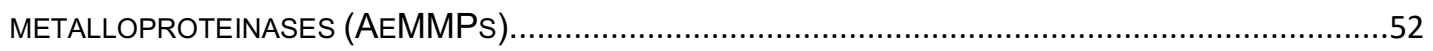

Figure 5. Determination of the tRanslational start Site of the AeMMP2 gene by 5'RACE....54

Figure 6. PhYLogenETIC RELATIONSHIPS OF AE. AEGYPTI MATRIX METALLOPROTEINASES (AEMMPS)

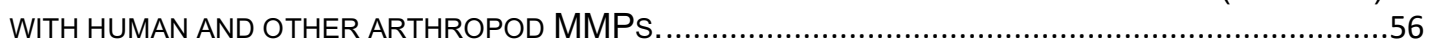

Figure 7. EXPRESSION PROFILES AEMMP1, AEMMP2, AEMMP4, AND AEMMP6 DURING DIFFERENT LIFE

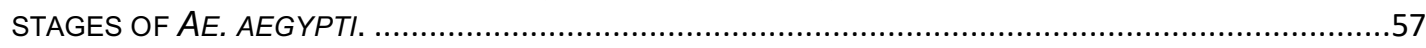

FiguRE 8. EXPRESSION PROFILES FOR AEMMP1, AEMMP2, AEMMP4, AND AEMMP6 IN MIDGUTS AND CARCASSES OF AE. AEGYPTI FEMALES AFTER A NON-INFECTIOUS OR CHIKV CONTAINING BLOODMEAL.

FiguRE 9. VALIDATION OF POLYCLONAL ANTIBODIES PAB-MMP1 AND PAB-MMP2. .61

Figure 10. Detection of AeMmP1 And AeMmP2 antigens by Western blot Analysis. .63

FiguRE 11. KINETICS OF RECOMBINANT AEMMP1 ACTIVITY IN AN IN VITRO MMP ACTIVITY ASSAY USING FS-6 AS SUBSTRATE.

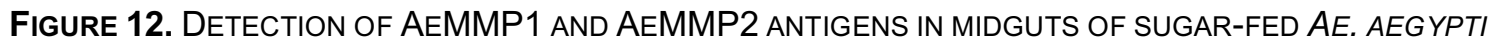
BY IFA.

FiguRE 13. DETECTION OF AEMMP1 AND AEMMP2 ANTIGENS IN MIDGUTS OF CHIKUNGUNYA VIRUS (CHIKV)-INFECTED AE. AEGYPTI BY IFA.

FiguRE 14. VISUAL COMPARISON OF BLOODMEAL DIGESTION OVER TIME IN THE MIDGUT OF AE. AEGYPTI.

FIGURE 15. ULTRASTRUCTURAL (SEM) IMAGING OF THE MIDGUT SURFACE OF AE. AEGYPTI. . .82

FIGURE 16. ULTRASTRUCTURAL (TEM) IMAGING OF MIDGUT CROSS-SECTIONS OBTAINED FROM FEMALE AE. AEGYPTI, WHICH HAD BEEN SUGARFED, BLOODFED OR ORALLY INFECTED WITH CHIKV (TITER IN THE BLOODMEAL: $10^{7}$ PFU/ML). . .85

FIGURE 17. BASAL LAMINA THICKNESS AND CHIK VIRION DENSITY AT THE BL .87 
FIGURE 18. THREE-DIMENSIONAL (3-D) RECONSTRUCTION FROM 108 STACKED FIB-SEM-GENERATED IMAGES SERIALLY SECTIONED AT 20-NM INTERVALS SHOWING MIDGUT TISSUE OBTAINED FROM A CHIKV INFECTED MOSQUITO AT 24 H POST-INFECTIOUS BLOODMEAL (PIBM).

FigURE 19. RARE EVENT OF AN INFECTED TRACHEA OBSERVED WITH TEM

FIGURE 20. ULTRASTRUCTURAL (TEM) EXAMINATION TO ASSESS HOW MULTIPLE BLOODMEALS AFFECT CHIKV DISSEMINATION FROM THE MIDGUT OF AE. AEGYPTI.

FIGURE 21. QUANTITATIVE DETECTION OF PLUS- AND MINUS-STRAND RNAS OF CHIKV AND MAYARO VIRUS (MAYV) VIA TAQMAN QRT-PCR ASSAYS.

FIGURE 22. SCHEMATIC REPRESENTATION OF CHIKV DISSEMINATION FROM THE MIDGUT OF AE. AEGYPTI.

FiguRE 23. PHYLOGENETIC ANALYSIS OF FULL-LENGTH RNA GENOMES OF GEOGRAPHICALLY DIVERSE MAYV STRAINS/ISOLATES.

FIGURE 24. INTENSITIES OF MAYV IQT AND TRVL INFECTIONS IN MIDGUTS AND CARCASSES OF AE. AEGYPTI STRAINS HWE AND ORL.

Figure 25. PREVALENCE OF MAYV IQT AND TRVL INFECTIONS IN MIDGUTS AND CARCASSES OF AE. AEGYPTI STRAINS HWE AND ORL.

FIGURE 26. INTENSITIES OF INFECTION AND PREVALENCE OF MAYV IQT AND TRVL IN HEAD TISSUES OF AE. AEGYPTI STRAINS HWE AND ORL AND PREVALENCE OF MAYV IQT AND TRVL IN HWE AND ORL SALIVA SAMPLES

FIGURE 27. INTENSITIES OF MAYV TRVL INFECTIONS IN MIDGUTS, CARCASSES, AND HEAD TISSUES OF AE. ALBOPICTUS (COMO), AND PREVALENCE OF THE VIRUS IN SALIVA SAMPLES.

FIGURE 28. EFFECTS OF MIXED INFECTIONS BETWEEN MAYV IQT AND CHIKV 37997 ON VIRUS PREVALENCE IN MOSQUITO SALIVA.

TABLE 1. PRIMERS USED FOR EXPRESSION PROFILING OF AEMMP GENES BY QRT-PCR, FOR 5 ' RACE OF THE AEMMP2 TRANSCRIPT, AND FOR RECOMBINANT (R) PROTEIN EXPRESSION OF AEMMP1 AND AEMMP2.

TABLe 2. OligonUCLeotide PRIMERS USED FOR TAQMAN QRT-PCR to DETECT CHIKV AND MAYV CDNAS.

TABLE 3. IDENTIFICATION AND DESIGNATION OF PUTATIVE MATRIX METALLOPROTEINASE (MMP) GENES IN THE GENOME OF AE. AEGYPTI BASED ON VECTORBASE SEARCHES.

TABLE 4. SIZES AND VOLUMES OF MIDGUTS OBTAINED FROM SUGARFED AND BLOODFED (IMMEDIATELY AFTER BLOODFEEDING) AE. AEGYPTI.

TABLe 5. AmINo ACID SUBSTITUtIONS SPECIFIC FOR MAYV StRAINS TRLV 4675, TRVL 15537, AND IQT 4235 . 


\section{ABBREVIATIONS}

aa

amino acid

AaMMP Aedes albopictus matrix-metalloproteinase

ADAM a disintegrin and metalloproteinase

a disintegrin and metalloproteinase with thrombospondin

ADAMTS motifs

Ae. Aedes

AeMMP Aedes aegypti matrix-metalloproteinase

AMPA 4-aminophenylmercuic acetate

An Anopheles

ATP Adenosine triphosphate

BL basal lamina

cDNA complementary deoxyribonucleic acid

CoMO Columbia, Missouri

CPE cytopathic effects

CS cysteine switch

Cx Culex

Dm Drosophila melanogaster

DMEM Dublecco's Modified Eagle Medium

DNA deoxyribonucleic acid

ECM extracellular matrix

EDTA ethylenediaminetetraacetic acid

EIP extrinsic incubation period

ER endoplasmic reticulum

FBS fetal bovine serum

FIB focused ion beam

h hours

HEPES 4-(2-hydroxyethyl)-1-piperazineethanesulfonic acid

$\mathrm{Hu}$ human

HWE Higgs White Eye

IPTG Isopropyl $\beta$-D-1-thiogalactopyranoside

JAK Janus kinases

MEM Minimum Essential Medium

MMP matrix-metalloproteinase

MT transmembrane linked

MTT 3-[4,5-dimethylthiazol-2-yl]-2,5-diphnyltetrazolium bromide

NC nucleocapsid 


$\begin{array}{ll}\text { NI } & \text { non-infectious } \\ \text { ORF } & \text { open reading frame } \\ \text { ORL } & \text { Orlando } \\ \text { pAb } & \text { polyclonal antibody } \\ \text { pbm } & \text { post blood meal } \\ \text { PBS } & \text { phosphate-buffered saline } \\ \text { PCR } & \text { polymerase chain reaction } \\ \text { pfu/ml } & \text { plaque forming units per ml } \\ \text { pibm } & \text { post-infectious bloodmeal } \\ \text { qPCR } & \text { quantitative polymerase chain reaction } \\ \text { qRT-PCR } & \text { quantitative Reverse transcription polymerase chain reactior } \\ \text { RACE } & \text { Rapid Amplification of cDNA Ends } \\ \text { RISC } & \text { RNAi silencing complex } \\ \text { RNA } & \text { ribonucleic acid } \\ \text { RT } & \text { room temperature } \\ \text { SEM } & \text { Scanning electron microscopy } \\ \text { spp. } & \text { species } \\ \text { STAT } & \text { Signal Transducer and Activator of Transcription proteins } \\ \text { TEM } & \text { Transmission electron microscopy } \\ \text { TIMP } & \text { tissue inhibitor of MMP } \\ & \\ & \\ \text { AcMNPV } & \text { Autographa californica Multicapsid nucleopolyhedrovirus } \\ \text { CHIKV } & \text { Chikungunya virus } \\ \text { DENV } & \text { Dengue virus } \\ \text { EEEV } & \text { Eastern equine encephalitis virus } \\ \text { JEV } & \text { Japanese encephalitis virus } \\ \text { MAYV } & \text { Mayaro virus } \\ \text { ONNV } & \text { O'nyong'nyong virus } \\ \text { RVFV } & \text { Rift Valley fever virus } \\ \text { SINV } & \text { Sindbis virus } \\ \text { SLEV } & \text { St. Louis encephalitis virus } \\ \text { WEEV } & \text { Western equine encephalitis virus } \\ \text { WNV } & \text { West Nile virus } \\ \text { YFV } & \text { Yellow fever virus } \\ \text { ZIKV } & \text { Zika virus } \\ & \end{array}$




\section{ABSTRACT}

The research work of this thesis has focused on the midgut escape barrier and the dissemination mechanism of alphaviruses in Ae. aegypti. Primarily, we identified and characterized Ae. aegypti MMPs that are seemingly involved in midgut $\mathrm{BL}$ remodeling during bloodmeal digestion. We identified nine Aemmp genes encoded in the genome of Ae. aegypti. For four of those MMPs, their expression profiles were assessed in the various life stages of the mosquito as well as in midgut and carcass tissues of CHIKV infected and non-infected mosquitoes. Based on antibody-specific detection, AeMMP1 and AeMMP2 were localized to midgut associated tissues. We also showed that AeMMP1 possesses collagenase activity in vitro. This suggests that the BL surrounding the midgut is a potential target of the enzymatic activity of AeMMP1.

Using FIB-SEM, we demonstrated for the first time that orally acquired mature $\mathrm{CHIK}$ virions exit the mosquito midgut by directly traversing the midgut $\mathrm{BL}$ during bloodmeal digestion. CHIK virions localized to the $\mathrm{BL}$ as early as $24 \mathrm{~h}$ pibm and were present in a high density until $32 \mathrm{~h}$ pibm. At $48 \mathrm{~h}$ pibm, CHIK virions were present in a lower density and remained so until a subsequent bloodmeal was ingested by the mosquito, at which point $\mathrm{CHIK}$ virions were once again associated with the strands of the BL. In SEM, disruptions in the BL adjacent to muscle tissue were also observed early after bloodmeal ingestion. These findings suggest that bloodmeal ingestion imposes changes to the $\mathrm{BL}$ structure, which then allows CHIKV to pass through. Furthermore, we showed that alphavirus dissemination from the midgut is not a continuous event during 
systemic infection of the mosquito; instead there is a relatively narrow time window, less than $48 \mathrm{~h}$ pibm, during which de novo synthesized virions are able to exit the midgut.

Lastly, we compared the vector competence of two strains of MAYV, which demonstrated the potential of this virus to be transmitted by urban mosquito vectors such as Ae. aegypti and Ae. albopictus. The MAYV strains IQT and TRVL were able, when orally acquired, to infect the midguts of both Ae. aegypti and Ae. albopictus and to disseminate in these mosquitoes to secondary tissues, eventually infecting the salivary glands and being released along with their saliva. CHIKV and MAYV co-infection resulted in similar efficiencies of cotransmission by Ae. aegypti. However, prior systemic infection with MAYV resulted in superinfection exclusion of CHIKV in saliva. Thus, two relatively closely related alphaviruses can antagonize each other in the same mosquito vector depending on the sequence of their acquisition and subsequent infection of their invertebrate hosts. 


\section{CHAPTER I}

\section{INTRODUCTION}

\section{Mosquitoes as vectors for arthropod-borne viruses}

There are over 3500 species of mosquitoes that have been classified into two subfamilies, Anophelinae and Culicinae $(1,2)$. Anopheles species are responsible for the spread of malaria (3) and o'nyong'nyong virus (ONNV; Togaviridae; Alphavirus) (4). Within the subfamily Culicinae, mosquitoes of the genera Aedes and Culex represent the majority of the species that transmit arthropod-borne viruses (arboviruses). Other genera such as Haemagogus also contain arbovirus vectors, which are typically not involved in urban disease cycles (5). Culex species are responsible for the transmission of West Nile virus (WNV, Flaviviridae; Flavivirus), Japanese encephalitis virus (JEV, Flaviviridae;

Flavivirus), St. Louis encephalitis virus (SLEV, Flaviviridae; Flavivirus), Western equine encephalitis virus ( WEEV, Togaviridae; Alphavirus), and Eastern equine encephalitis virus (EEEV, Togaviridae; Alphavirus) to name a few (6-9). These viruses are mainly neurotrophic, infecting components of the nervous system and are known to cause encephalitis in the vertebrate host as they are aptly named (10).

The typical transmission cycles for these viruses involve birds as amplifying hosts and humans or equids as dead end hosts, which can develop severe disease symptoms by producing only a low viremia (11). Aedes transmit yellow fever virus (YFV, Flaviviridae; Flavivirus), dengue virus (DENV, 
Flaviviridae; Flavivirus), chikungunya virus (CHIKV, Togaviridae; Alphavirus), and Zika virus (ZIKV, Flaviviridae; Flavivirus) (12-16). In typical sylvatic transmission cycles, these viruses circulate between non-human primates as the main animal reservoir and Aedes as the vector. However, in an urban cycle, these viruses can utilize human beings as amplifying hosts as they typically produce a very high viremia upon infection (17). A majority of viruses transmitted by Aedes species cause arthralgia with symptoms able to persist and often diagnosed as chronic disease (18-20).

Not all arboviruses are mosquito-borne. Flaviviruses and bunyaviruses can be transmitted by ticks, however, all alphaviruses are mosquito-borne $(21,22)$. Efficient spread of an arbovirus requires the presence of the arthropod vector and the vertebrate amplification host (reservoir) for the virus. This means that arbovirus ecology is dependent on vector ecology (23). Therefore, it is important to identify the habitats of specific mosquito vectors in a given region and limit vector abundance in order to prevent the spread of arboviral diseases. A major problem is the geographic distribution of Aedes spp. $(24,25)$. Therefore it is important to have an understanding of how these viruses interact in the mosquito vector so we can develop new tools and disease prevention strategies.

Chikungunya virus (CHIKV) is transmitted by the bite from an infected female mosquito. Ae. aegypti, commonly referred to as the yellow fever mosquito, and Ae. albopictus, the Asian tiger mosquito, are recognized as the principal vectors for the virus (26). Ae. albopictus are native to tropical and subtropical areas of Southeast Asia but are now considered an invasive species 
across Europe and the Americas believed to be introduced by the import of used tires from Asia containing eggs that hatched upon rainfall $(25,27)$. Ae. aegypti originates from Africa but is now present in tropical and subtropical regions of the world. Both species have been identified in the southeastern United States $(28,29)$.

Mayaro virus (MAYV, Togaviridae; Alphavirus) is naturally transmitted by Haemagogus janthinomys (30). These mosquitoes are predominantly forest dwelling and reside mainly in Central and South America. As opportunistic feeders Haemagogus have been shown to feed mainly on birds and rodents, but are also known to take bloodmeals from humans (31). The threat for human disease outbreaks caused by MAYV stems from the ability of the virus to be transmitted by highly anthropophagic and urban setting mosquitoes like Ae. aegypti and Ae. albopictus (32).

Mosquitoes require both sugar meals and bloodmeals to survive and reproduce. They have a digestive system capable of storing both food types. When a sugar meal is acquired it is directed to the crop where it can be stored and later digested in the midgut (33). This prevents the midgut from being filled by a sugar meal and allows bloodmeals to be readily digested by the midgut. The purpose of a mosquito acquiring a bloodmeal is to enable the production of vitellogenin in fat bodies which is later transported to the ovaries and absorbed by oocytes where it is required for egg development (34).

Unlike wetland mosquito species that oviposit and develop in large habitats that are easy to locate, Aedes are known to utilize small artificial 
containers and tree holes for early stages of development $(35,36)$. This enables these mosquitoes to easily adapt to the urban human environment. Both $A e$. aegypti and Ae. albopictus are known to be aggressive biters and will bite throughout the day unlike other mosquito species known to be most active during dusk and dawn (37). Ae. albopictus typically feeds on mammals although this species is a very opportunistic biter, also feeding on avian hosts or even reptiles $(38,39)$. In contrast, Ae. aegypti preferentially feeds on humans and to a lesser extent on bovine, swine, rats, and chickens.

\section{Vector competence of mosquitoes}

Vector competence is defined as the intrinsic ability of arthropods to become infected, maintain, and transmit microbial agents. Not all mosquito species are competent vectors for every arbovirus; the vast majority of mosquitoes species are not even vectors at all. Genetic determinants of the vector play a critical role in its competence for arboviruses (40). For example, based on quantitative trait loci, it has been shown that genes associated with a mild midgut infection barrier (MIB) in Ae. aegypti aegypti (Puerto Rico) allowed persistent DENV2 infection in $65 \%$ of individuals. However, the genetic makeup of Ae. aegypti formosus (Nigeria) resulted in a strong MIB allowing only $11 \%$ of individuals to get infected with the virus (41). Another genetic determinant of vector competence are the innate immune responses to arbovirus infection $(42,43)$ This has been demonstrated by generating Ae. aegypti that activate the conserved JAK/STAT pathway in fat body tissue during DENV2 and DENV4 
infection (44). Sindbis virus (SINV) infection of the midgut can be affected by manipulating RNAi pathways in the midgut (45). The differences in immune response might be responsible for the different infection patterns of CHIKV in two Ae. aegypti strains, HWE and ORL $(14,45,46)$.

Environmental factors such as temperature are also important determinants of vector competence. Ae. albopictus from Germany were susceptible to $\mathrm{ZIKV}$ at $27^{\circ} \mathrm{C}$ but not at lower temperatures, for example $18^{\circ} \mathrm{C}$ (47). Also in field situations it is important to keep in mind that mosquito populations of the same species can be highly heterogenic, consisting of individuals that differ in their genetic background, age, reproductive status, size, and nutritional status. This heterogeneity is known to drastically effect the overall vector competence of a population as some individuals may be highly susceptible to a particular arbovirus, allowing the virus to generate high titers, whereas other individuals within the same population may not become infected at all (48). Older individuals in Ae. aegypti populations have been shown to have weaker immune systems (49). Diet is also important in early life stages. Larvae that are starved have been shown to show a decrease in immune gene expression as mature mosqutioes (50).

Closely related to vector competence is the vectorial capacity of mosquito species. Estimates of vectorial capacity account for multiple factors that play a role in the association of a vector with a pathogen that can be transmitted and a susceptible vertebrate host (51). One such factor is the lifespan of a mosquito. Ae. aegypti have been shown to live as mature mosquitoes for around 35 days in 
nature, and can be infective for up to 23 days under optimal conditions (52). The time it takes for a vector to ingest a viremic bloodmeal until infectious virus particles are present in the saliva is known as the extrinsic incubation period (EIP) (53). Once the EIP for the virus has been completed, a female mosquito is able to transmit the virus for the remainder of her life (54). In urban settings, mosquitoes such as Ae. aegypti have adapted to biting populations in close proximity, ingesting multiple bloodmeals, and they prefer bloodmeals over sugar meals $(38,55-57)$. Different viruses possess different EIPs. The average EIP of DENV4 in Ae. albopictus and Ae. aegypti has been shown to be around 5-6 days $(58,59)$ while the EIP of CHIKV in Ae. aegypti can be as short as 2 days. Further, analysis of the biting behavior of these mosquitoes showed that secondary and tertiary bites posed the highest chance for the successful transmission of either virus $(14,60)$.

Lifespans and feeding behaviors of mosquitoes critically influence their overall vectorial capacity; however, a population of susceptible vertebrate hosts must be abundantly present for an arboviral disease to spread. This is demonstrated well by MAYV. Aedes species are believed to be capable vectors for MAYV in the urban environment. Regardless, the virus has so far been isolated only in rural and rain forest areas of Central and South America where Haemagogus species are highly abundant but not Ae. aegypti (32,61-63). Additionally, MAYV infection in humans is characterized by a low titer and short virema of around 3 days (64). Therefore humans might not be an amplifying host. Unlike WNV which utilizes birds in proximity to urban environments MAYV is 
known to infect non-human primates which are not present in urban areas $(65,66)$. This suggests MAYV will need to adapt to amplify in humans in order to raise the vectorial capacity of urban mosquito species such as Ae. aegypti.

\section{Chikungunya and Mayaro viruses}

CHIKV and MAYV both belong to the Semliki Forest virus antigenic complex within the genus Alphavirus of the family Togaviradae (22).

Alphaviruses are enveloped viruses that are spherical in shape with a diameter of 60-70 $\mathrm{nm}$ (67). Their genome consists of a $\sim 12 \mathrm{~kb}$ single stranded positive sense RNA molecule which is capped at its $5^{\prime}$ end and contains a polyadenylated tail at its 3 ' end. The viral RNA contains two open reading frames (ORFs) (68) (Fig. 1). The first ORF begins at the 5' end and encodes the four nonstructural proteins, nsP1, nsP2, nsP3, and nsP4 (69). The second ORF is under the control of a subgenomic promoter located around 7400 nucleotides downstream of the viral RNAs' 5' end (68). The second ORF encodes the structural proteins C, E3,E2 6k/TF, and E1 (Fig. 1) (70).

\section{Epidemiology}

CHIKV and MAYV are both emerging arboviruses. CHIKV is an Old World alphavirus that was first discovered in Tanzania, Africa in 1952 (71). The virus has recently caused severe outbreaks in 44 countries and territories throughout Central and South America including the Caribbean with over 1.3 million suspected cases in the Western Hemisphere as of April 2015 (72). 

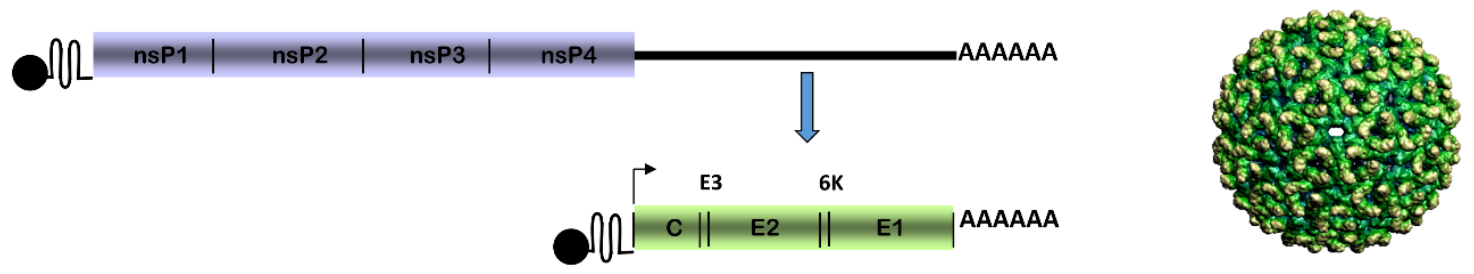

Figure 1. Genome structure of Alphaviruses.

Alphavirus form spherical enveloped virions $70 \mathrm{~nm}$ in diameter with a single stranded positive sense RNA genome. The genomes are approximately 12,000 nt and contain a 5'cap and a poly A tail. The genome organization consists of 4 non-structural proteins followed by subgenomic RNA that encodes for the structural proteins $\mathrm{C}$ and the glycoproteins E3, E2, 6k/TF, and E1. 
MAYV is a New World alphavirus that was first isolated in Trinidad in 1954 (73). In recent years, MAYV infection human cases have been reported in Peru, Bolivia, Brazil, and Haiti $(62,74,75)$. MAYV presence in the Americas suggests that it could imitate the epidemiological evolution of other arboviruses like ZIKV and become a major threat to public health. In 2015, MAYV was isolated from a Haitian infant who was co-infected with dengue virus serotype 1 (75). Haiti is known to be a habitat for Ae. aegypti suggesting that this species could be involved in local transmission (28). It also raises concerns about Ae. aegypti's ability to co-transmit multiple arboviruses during feeding on a human host and how a co-infection of multiple arboviruses can affect virus dissemination and replication in a mosquito $(32,76)$.

\section{Disease}

In human patients suspected of arbovirus infection the term "ChikDenMaZika syndrome" has been suggested due to the common symptoms associated with CHIKV, DENV, MAYV, and ZIKV (77). These symptoms include fever, headache, muscle pain, joint swelling, joint pain, and rash. The joint pain is known to be debilitating and can render a patient bed ridden $(78,79)$. Both $\mathrm{CHIKV}$ and MAYV can cause acute, subacute, or chronic disease $(80,81)$. In an acute infection MAYV presents with a short viremia of 3-4 days followed by a 7-12 day period in which the common symptoms become evident (82). CHIKV patients exhibit a high viremia for $\sim 7$ days and will present with common symptoms 3-7 days after an infectious bite (83). In a subacute infection joint pain and swelling 
can persist for a few weeks, and in chronic infections patients can experience these symptoms for months for both CHIKV and MAYV $(19,20)$.

There is currently no vaccine available for CHIKV or MAYV. Patients are offered supportive care targeting pain and fever reduction using nonsteroidal anti-inflammatory drugs (NSAIDs) $(64,84)$. NSAIDs have been used in conjunction with steroids and low dose aspirin, however this treatment is not recommended due to the high risk of upper gastrointestinal bleeding (85). The antiviral ribavirin has been tested in recent studies and is shown to be effective in treatment of CHIKV arthralgia (86).

\section{Virus replication}

Both CHIKV and MAYV enter the cell through receptor mediated endocytosis moderated by the E2 glycoprotein (87). Following cell entry, alphavirus virions are enclosed in early/late endosomes in which the change in $\mathrm{pH}$ to a slightly acidic environment triggers E1-E2 heterodimer destabilization. The E1 glycoprotein contains a fusion loop that joins the late endosomal membrane allowing the nucleocapsid (NC) to be released into the cytoplasm (88). Once in the cytoplasm, the NC is trafficked to the ER where interaction with ribosomes enables disassembly and release of the genomic RNA (89). The nonstructural proteins are immediately translated from the genomic RNA into the polyprotein P1234 (90). Most alphaviruses including those used in this study, CHIKV 37997, MAYV IQT 4235, and MAYV TRVL 4675, will produce two polyproteins, an incomplete P123 major product, and a full length P1234 minor 
product $(68,91)$. This is due to the presence of an opal stop codon located at the end of nsP3 that is read through during translation. Following translation of $\mathrm{P} 1234, \mathrm{nsP} 2$ is proteolytically active in the polyprotein with the ability to cleave in cis at the nsP3/nsP4 junction (92). Therefore, polyprotein processing is regulated during infection as nsP2 can only cleave other sites within the polyprotein in trans (93). The cleaved nonstructural proteins will assemble to form a replication complex that will generate both the negative-sense intermediate (only present during replication serving as a template strand), and positive-sense genomic RNA via the RNA-dependent RNA polymerase (RdRP) activity of nsP4 (94). $\mathrm{NsP} 3$ is required along with nsP4 to generate the second ORF that encodes the structural proteins, which are transcribed form the negative-sense RNA (95). NsP1 is membrane- associated and contains guanine-7-methyltransferase and guanylyltransferase enzymatic activity which is responsible for capping and methylation of both genomic and subgenomic RNAs $(96,97)$.

\section{Nucleocapsid and virion assembly}

The structural proteins are translated as a polyprotein with the capsid protein being cleaved via autoproteolysis (90). The cleaved amino acid residues 81-112 of capsid recognize an encapsidation signal present on the genomic RNA (98). This nucleation event will allow for 240 copies of capsid and a single copy of the positive-sense genomic RNA to form the NC. The NC has an icosahedral symmetry of $\mathrm{T}=4$ and a diameter of $\sim 40 \mathrm{~nm}(90,99,100)$. With the capsid protein cleaved, the $\mathrm{N}$-terminus of the structural polyprotein now encodes an 
endoplasmic reticulum (ER) signal peptide that will localize the polyprotein across the ER membrane for further processing. The polyprotein will be transported to the plasma membrane via the trans-Golgi network (68). While in transport furin cleavage is responsible for the separation of PE2 into E3 and E2 with E3 not being required for infectious particle formation $(90,101)$. E1 and E2 will then form heterodimers. The glycoproteins are trafficked to the plasma membrane separately from the NC (102). Once both the NC and glycoproteins have joined each other for final assembly, the de novo synthesized virion will bud from the plasma membrane. The virion now possesses an envelope with 80 glycoprotein spikes consisting of trimers of E2 and E1 heterodimers responsible for receptor detection (103). Although budding is known to result in an infectious particles release from mammalian cell types, alphaviruses are also known to bud into internal vesicles before release from the arthropod cell $(68,104,105)$.

\section{Mosquito infection}

In order for a mosquito to transmit an arbovirus to an uninfected vertebrate host the vector must be systemically infected (54). To accomplish this a virus must overcome innate immune responses, disseminate through different tissue barriers ultimately infecting the salivary glands to be released into the bloodstream of a vertebrate along with saliva during probing/biting (Fig. 2). Upon the uptake of a viremic bloodmeal, the virus is trafficked into the midgut lumen (106). Once inside the lumen, the virus will infect a midgut epithelial cell through its microvilli via receptor-mediated endocytosis $(107,108)$. The virus has a limited 
time window to infect the cell as the peritrophic membrane will begin to form as soon as 4-hours (h) post-infection (109). This membrane is an arrangement of chitin microfibrils secreted from the epithelium that surrounds the bloodmeal to protect the epithelium (110) and allow small molecules to be absorbed by the midgut after trypsin-like serine proteases have broken down the ingested meal $(111,112)$.

The midgut is the first mosquito tissue to be infected by an arbovirus. It is tubular in shape and can be divided into three compartments, the foregut, the midgut, and the hindgut. The foregut contains the esophagus, crop, proventriculus, and a cardiac valve with the purpose to prevent midgut contents from leaking back into the foregut and crop (113). The posterior portion of the midgut along with the hindgut play major roles in sugar meal and water absorption and the midgut is involved in nutrient absorption and bloodmeal digestion (114). The intake of a bloodmeal drastically changes the structure of midgut epithelial cells. Nuclei are shown to condense, mitochondria enlarge, and the rough ER grows in size as well while the basal labyrinth shrinks near the basal side of the cell (115). After an alphavirus has entered the epithelial cell, virus replication will begin to occur as described above and newly synthesized virions need to disseminate from the midgut.

\section{Basal lamina}

The mosquito midgut contains a single layer of epithelial cells that is surrounded by a multi-stranded extracellular matrix known as the basal lamina 


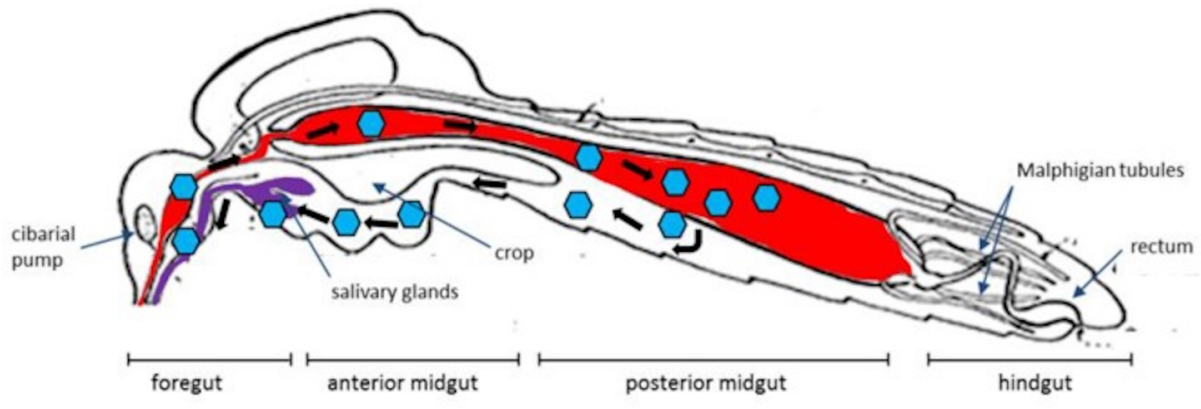

Figure 2. Pathway of arbovirus dissemination in Ae. aegypti.

Representation of arbovirus tropism in a mosquito vector. Viruses first infect the midgut after a viremic bloodmeal and must disseminate into secondary tissues, eventually infecting the salivary glands. Virions are represented by blue hexagons. Adapted from Franz, Alexander W E et al. "Tissue Barriers to Arbovirus Infection in Mosquitoes." Viruses 7.7 (2015): 3741-3767. 
A

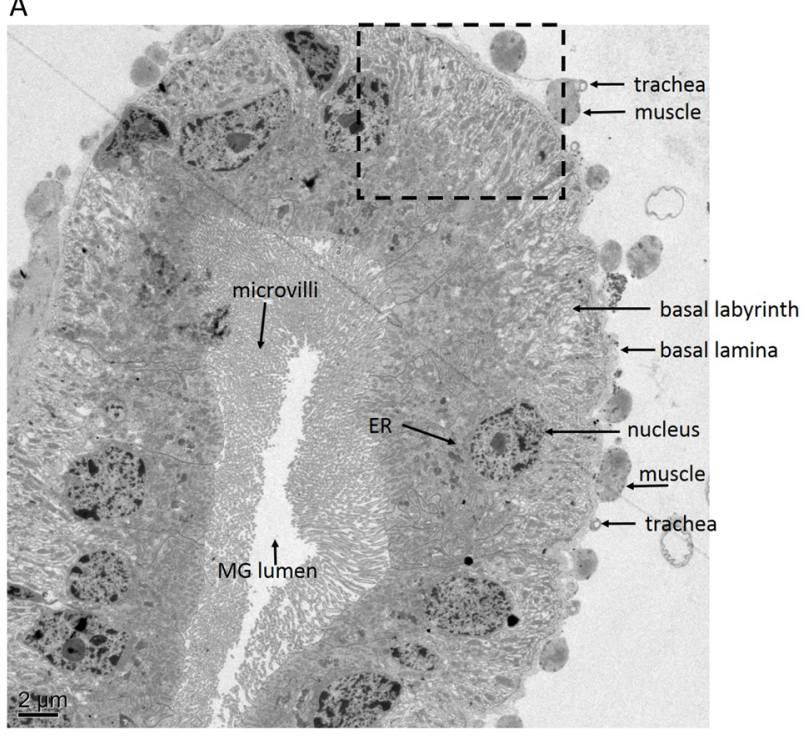

B

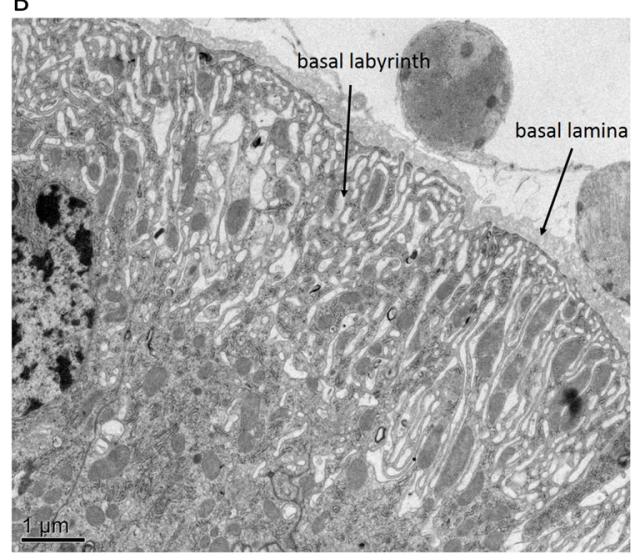

Figure 3. Ultrastructural views of the posterior midgut tissue of Ae. aegypti.

(A) Cross-section of a non-infected midgut of a female at 7 days post-bloodmeal (magnification: 600x). (B) Close-up view of image in panel

(A) at 2000x magnification. Note the structured basal lamina surrounding the midgut. $\mathrm{MG}=$ midgut. Adapted from Franz, Alexander W E et al. "Tissue Barriers to Arbovirus Infection in Mosquitoes." Viruses 7.7 (2015): 3741-3767. 
(BL) (Fig. 3). The anterior of the BL is densely packed with crosslinked proteins, mainly laminin and collagen IV which are secreted form the epithelial cells $(116,117)$. The insect $B L$ functions as a barrier to macromolecules, provides a basis for the structural integrity for the midgut epithelium and separates the epithelium from the hemolymph and hemocoel (118). This tissue has been proposed to be a physical barrier of virus dissemination form the midgut as it has a size exclusion limit of around $10 \mathrm{~nm}$ which would prevent CHIKV and MAYV virions with a diameter of 60-70 $\mathrm{nm}$ from freely passing through (119-121).

In this dissertation, we will address two possible explanations for how alphaviruses are able to disseminate through this midgut escape barrier. The first is that the mosquito midgut possesses enzymatic activity that could be responsible for $\mathrm{BL}$ alterations that allow the virion to traverse the $\mathrm{BL}$. The second is that the $B L$ is a dynamic structure that has plasticity, which can be modified under stressful events.

\section{Potential role for matrix metalloproteinases in BL remodeling}

In vertebrates, metalloproteinases are the most important enzymes responsible for extracellular matrix (ECM) remodeling (122). Metalloproteinases are grouped into two major families of zinc-dependent endopeptidases: matrix metalloproteinases (MMPs) and a disintegrin and metalloproteinase (ADAM)/ADAM with thrombospondin motifs (ADAMTS) proteinases. In humans, endopeptidases of the ADAMs family have been implicated in the control of membrane fusion, cytokine and growth factor shedding, and cell migration, as 
well as processes such as muscle development, fertilization and cell fate determination (123). MMPs cleave a wide range of ECM proteins (including collagenase IV and laminin), degrade intercellular junctions, generate cleavage products that act as novel signaling molecules and/or modify the action of latent or active signaling molecules thereby changing cell and tissue physiology (124). Specifically, MMPs are essential for connective tissue remodeling during embryonic development, angiogenesis, bone growth and healing of wounds (125-127). Vertebrate MMPs are grouped according to substrate specificity into collagenases, matrilysins, stromelysins and gelatinases, the latter two degrading laminin and/or collagen IV (128). Another classification scheme distinguishes between 'membrane-type' MMPs (MT-MMPs) and 'other' MMPs (129).

MMPs are not restricted to vertebrates; they also have been described and analyzed in plants such as Arabidopsis thaliana (130) and tomato (131), in the nematode Caenorhabditis elegans (132) and in insects such as Drosophila melanogaster, Manduca sexta, Tribolium castaneum and the Plasmodium parasite vector, Anopheles gambiae (133-140). In insects, MMP activity is required for wound healing and tissue rearrangements during molts and metamorphosis involving ECM degradation $(135,137,138,141,142)$. Furthermore, insect MMPs have been shown to be involved in innate immunity responses to tissue invasion by pathogens $(137,140,143)$. For Ae. aegypti, MMPs and their possible roles in biological processes have not been described so far. 


\section{Midgut trachea}

Another hypothesized route of dissemination for arboviruses is through tracheal cells that extend into the midgut $(144,145)$. Invertebrates have an open respiratory system that encompasses a complex network of tubular channels responsible for oxygen and gas exchange to various tissues of the animal (146). Spiracles from the surface of the arthropod condense into trachea, becoming smaller and smaller ending in chitinous tubes known as tracheoles (147). Many alphaviruses including SINV, WEEV, CHIKV, and the bunyavirus Rift Valley Fever virus (RVFV) have been shown to infect midgut associated trachea and tracheoles after intrathoracic injection and after oral acquisition of the virus (148152). Tracheae are also closely associated with muscle fibers surrounding the midgut and both are incorporated in the outer layers of the $B L(151)$. These results support the possibility that arboviruses disseminate from the midgut and through the BL using tracheal cells as conduits; however, this is not a definite and further investigation is required.

\section{Salivary gland infection}

A critical organ that an arbovirus must infect prior to transmission to a new vertebrate host is the salivary glands. In mosquitoes such as Ae. aegypti, the salivary glands consist of two pairs of glands. Each gland has two lateral lobes and one medial lobe, which are connected to a main salivary duct (14). CHIKV has been detected in salivary glands of Ae. aegypti as early as 2 days postinfectious bloodmeal (pibm) and the virus produced median titers of $10^{4} \mathrm{pfu} / \mathrm{ml}$ in 
this tissue as early as 4 days pibm (14). Although infection rates of salivary glands may reach $100 \%$ in mosquitoes following oral acquisition of the virus, typically only $60 \%$ of those infected mosquitoes had detectable CHIKV in their saliva at 7 days pibm (14). This suggests that there is a salivary gland escape barrier for CHIKV as has been described for SINV, and RVFV $(153,154)$, and that it is possible that this escape barrier is also present for other related alphaviruses such as MAYV. Once the virus has been released into her saliva, a female mosquito is systemically infected and able to transmit the virus for the remainder of her life (52).

\section{Immune responses to alphaviruses in mosquitoes}

The RNA interference (RNAi) pathway is the principal immune pathway responding to the presence of replicating arboviruses in mosquitoes (155). Viral genome-derived long dsRNA is the trigger for antiviral RNAi. Dicer2 senses the presence of long dsRNAs in a cell and processes those into $21 \mathrm{bp}$ duplexes (156). With the help of R2D2, these 21 bp duplexes are unwound and one strand, the guide strand, is inserted into the RNAi silencing complex (RISC) (157), whose central catalytic component is Ago2 (158). Following guidance of RISC to long (viral, single-stranded) RNAs with perfect sequence homology, Ago2 then cleaves the target RNA resulting in destruction of viral genomes (159). In any naturally arbovirus-infected mosquito, RNAi is sensing the presence of viral RNA genomes during replication and, to a certain extent, is destroying those. However, arboviruses including alphaviruses have developed strategies to 
cope with the RNAi response to prevent their complete elimination (160). Thus, there seems to be a fine balance between the RNAi response of the vector and viral countermeasures allowing a virus to survive in the vector. The gatekeeper role of RNAi in the mosquito has been experimentally demonstrated. RNAi impairment in Ae. aegypti causes increases in arbovirus titers in midgut tissue, and allows for a more rapid dissemination to secondary tissues resulting in shorter EIPs $(45,161,162)$. Complete RNAi inhibition can cause replicating viruses to reach lethal concentrations in the insect, which would be detrimental for both, the parasite and its invertebrate host $(163,164)$. Conversely, priming the RNAi response pathway in transgenic mosquitoes resulted in complete elimination of DENV2 from midgut tissue (165). This manufactured midgut infection barrier demonstrates the importance of unraveling the mechanisms of dissemination in the mosquito.

RNAi is not the only immune pathway of $A$ e. aegypti that arboviruses must compete with. The Toll pathway is activated by a cytokine known as Spaetzle that triggers a signaling cascade through the toll membrane receptor (166-168). Downstream the transcription factor Rel I is transported to the nucleus and initiates transcription of antimicrobial peptides $(169,170)$. Inhibition of DENV by the naturally occurring bacteria Wolbachia occurs through this immune pathway via transcription of cecropin and defensin (171-173). SINV infection is also known to activate this pathway leading to the transcription of Dif (174). West Nile virus is known to overcome this pathway by down-regulating Spaetzle upstream of Toll receptor activation, thus preventing Rel I to become active (168). 
The immune deficiency (IMD) pathway is also active in Ae. aegypti. Pathogen recognition receptors (PRRs) recognize pathogen associated molecular patterns (PAMPs) and can activate the JNK signaling pathway or activate the transcription factor Rel 2 to generate antimicrobial effectors $(170,175)$. These pathways are shown to be active in both DENV and SINV infections $(167,176)$. Also, inhibition of the IMD pathway in Ae. aegypti strains that are refractory to DENV increases midgut titers during infection (177).

The Janus kinase (JAK)- signal transducer and activator of transcription protein (STAT) pathway is yet another immune pathway present in Ae. aegypti. This immune pathway was first identified as an interferon induced pathway in vertebrates, which is conserved in insects (178). This pathway is activated when the transmembrane receptor, Dome, binds a ligand and dimerizes. This activates JAKs downstream which will recruit STAT proteins that dimerize and translocate to the nucleus and begins the production of effector proteins $(170,179)$. This pathway is active in DENV infection of Ae. aegypti and is also active in ONNV in Anopheles $(179,180)$. With enough understanding of these immune pathways we can discover novel ways to control virus infections of these vectors and generate new tools to aid in public health.

\section{Purpose}

It is important to take multiple approaches when attempting to prevent potential public health epidemics. Both CHIKV and MAYV are emerging arboviruses causing disease in tropical regions of the world. One major approach 
is to study the infection pattern in vertebrate hosts and identify potential vaccine candidates. However, another approach that can yield the same results in terms of disease prevention is to study these viruses in the vector Ae. aegypti. Studying the infection in vivo gives an advantage because disease prevention strategies can be tested in the organism it will be implemented in. Before these strategies can be developed, we need to acquire knowledge about dissemination mechanisms and infection patterns of alphaviruses in Ae. aegypti. Therefore the focus of this research is to understand how an alphavirus disseminates from the primary tissue of infection, the midgut, into secondary tissues enabling transmission to a new vertebrate host. Here we identify potential proteases involved in CHIKV midgut escape, as well as identify the time window and route of dissemination for alphavirus midgut escape after a viremic bloodmeal. We also describe the infection pattern of another alphavirus MAYV, and examine the potential of a mosquito to become co-infected and the potential for alphavirus cotransmission. 


\section{CHAPTER II}

\section{MATERIALS AND METHODS}

\section{Mosquitoes}

Ae. aegypti of the Orlando ("ORL") and Higgs White Eye ("HWE") strains, the latter exhibiting eye-pigment deficiency, were reared and maintained in a $\mathrm{BSL} 2$ insectary at $28^{\circ} \mathrm{C}, 75-80 \%$ relative humidity, and a $12 \mathrm{~h}$ light $/ 12 \mathrm{~h}$ dark cycle. An Ae. albopictus strain designated "CoMO" was established in 2016 from a locally captured female (Columbia, MO, USA), which tested positive for Wolbachia infection via a PCR assay (data not shown). In colony, Ae. albopictus were reared under the same conditions as Ae. aegypti HWE and ORL. For colony maintenance, mosquitoes received artificial bloodmeals consisting of defibrinated sheep blood (Colorado Serum Company, Denver, CO, USA). Raisins were provided to the adult mosquitoes as a sugar source.

Ae. albopictus CoMO were hatched in distilled water following a deoxygenation step to generate higher hatch rates. Therefore, a $1 \mathrm{~L}$ mason jar was filled with $750 \mathrm{~mL}$ of distilled water and autoclaved. Immediately after removal from the autoclave, the lid was sealed tight and allowed to cool to establish a vacuum and deoxygenate the water. Egg papers were placed in the water immediately after the vacuum was broken and allowed to hatch for $3 \mathrm{~h}$. 


\section{Virus strains}

CHIKV strain 37997 (West African genotype; GenBank accession: AY726732.1) from Senegal, was used in this study. This virus was isolated in 1983 and passaged once in AP-61 cells (Ae. pseudoscutellaris) and two times in Vero cells (African green monkey kidney). We prepared virus stocks by passaging the virus in Vero cells and collecting supernatant at $24-30 \mathrm{~h}$ postinfection; titers in Vero cells reached $6.6 \times 10^{6} \mathrm{pfu} / \mathrm{ml}$.

MAYV strain IQT 4235 from Loreto, Peru (GenBank \#: MK070491) and the type strain TRVL 4675 from Trinidad (GenBank \#: MK070492) were also used in this study. Both viruses are considered low passage strains. The IQT 4235 strain was isolated in 1997 and has been passaged once in C6/36 (Ae. albopictus) cells and once in Vero cells before being used in this study. We prepared virus stocks by passaging the virus twice in Vero cells. At $30 \mathrm{~h}$ and $39 \mathrm{~h}$ post-infection, titers in in Vero cells reached $4.6 \times 10^{6} \mathrm{pfu} / \mathrm{ml}$ and $3.3 \times 10^{6} \mathrm{pfu} / \mathrm{ml}$, respectively. The TRVL 4675 prototype strain was isolated in 1954 and has been passaged in BHK21 and Vero cells before use in this study (Anderson et al. 1957). Virus stocks were prepared via passage in Vero cells in which titers reached $6.0 \times 10^{6} \mathrm{pfu} / \mathrm{ml}$ at $30 \mathrm{~h}$ post-infection.

\section{Oral virus infection of mosquitoes}

Prior to mosquito infections all viruses were propagated in Vero cells seeded in T-25 flasks at a multiplicity of infection of 0.01 using Dulbecco's 
Modified Eagle Medium (DMEM) supplemented with 7\% fetal bovine serum (FBS). For oral challenge of mosquitoes with CHIKV and MAYV, virus-containing cell culture supernatant was collected at $24-30 \mathrm{~h}$ and $30-39 \mathrm{~h}$ post infection, respectively, and mixed with defibrinated sheep blood at a 1:1 ratio including the addition of $10 \mathrm{mM}$ ATP. Seven-day-old females that had been (sugar-)starved overnight were fed an artificial bloodmeal containing virus-infected cell culture/blood mixture or non-infected cell culture/ blood mixture (control) for $1 \mathrm{~h}$ at $37^{\circ} \mathrm{C}$ using a single glass feeder/carton lined with hog intestine. After screening, fully engorged females were maintained in $1.9 \mathrm{~L}$ cartons and offered raisins and water until further analysis. All virus infections were carried out in a biosafety level 3 laboratory within the Laboratory for Infectious Disease Research (LIDR) at the University of Missouri.

\section{Virus detection by serial sample dilutions in plaque assays}

Virus titers from individual mosquitoes, midguts, carcasses, and head tissues were determined by plaque assays in green monkey kidney (Vero) cells. After dissection tissue samples from individual mosquitoes were homogenized in $1 \mathrm{ml}$ sample processing buffer (DMEM with 7\% FBS and 1\% 4-(2-hydroxyethyl)1-piperazineethanesulfonic acid [HEPES]). Homogenized samples were sterilefiltered using Acrodisc HT Tuffryn $0.2 \mu$ m syringe filters (Pall Life Sciences, East Hills, NY, USA). Vero cells were seeded onto 24-well plates and incubated for two days at $37^{\circ} \mathrm{C}$ to achieve $95-100 \%$ confluence. Cells were infected with $10-$ fold serial dilutions of each mosquito homogenate. Cells were incubated for $1 \mathrm{~h}$ at 
$37^{\circ} \mathrm{C}$ before being overlaid with an agarose nutrient mixture [1x Medium 199 (Sigma-Aldrich, St. Louis, MO, USA), 10\% FBS, 4\% $\mathrm{NaHCO}_{3}, 0.5 \%$ Minimum Essential Medium (MEM) vitamins, 0.5\% MEM amino acids (Mediatech Inc., Manassas, VA, USA)]. After incubation at $37^{\circ} \mathrm{C}$ for 2 days for $\mathrm{CHIKV}$ and 3 days for MAYV, cells were stained with MTT (3-[4,5-dimethylthiazol-2-yl]-2,5diphnyltetrazolium bromide) (Sigma-Aldrich) and incubated at $37^{\circ} \mathrm{C}$ for $24 \mathrm{~h}$ before counting the number of plaques for each sample. Viral titers of individual mosquitoes were calculated as plaque forming units per $\mathrm{ml}(\mathrm{pfu} / \mathrm{ml})$.

\section{RNA extraction with TRIzol reagent}

Groups of 10 larvae, pupae, whole-body mosquitoes, midguts, carcasses, or saliva samples were collected for total RNA extraction using TRIzol reagent (Invitrogen, Carlsbad, CA, USA). Tissues were homogenized with a hand held motor and RNase Zap (Thermo Fisher, Kalamazoo, MI, USA) treated pestles before adding TRIzol reagent to a final volume of $1 \mathrm{ml}$. The homogenate was incubated at room temperature (RT) for $5 \mathrm{~min} .200 \mu \mathrm{l}$ of chloroform was added to the $1 \mathrm{ml}$ of TRIzol and mixed, followed by a 3 min incubation at RT. Samples were then centrifuged at $12,000 \times g$ at $4^{\circ} \mathrm{C}$ for $15 \mathrm{~min}$. The colorless aqueous phase was separated into a new tube, and for saliva samples $5 \mu \mathrm{g}$ of RNase-free glycogen was added as a carrier. $500 \mu \mathrm{l}$ of RT isopropanol was added to the aqueous phase and incubated for $10 \mathrm{~min}$, thereafter samples were centrifuged at $12,000 \times g$ for $10 \mathrm{~min}$ at $4^{\circ} \mathrm{C}$. The supernatant was removed without disturbing the RNA pellet, and the pellet was washed with $1 \mathrm{ml}$ of $75 \%$ ethanol. The RNA 
pellet was then allowed to air dry before being resuspended in nuclease-free water.

\section{Database searches and phylogenetic analysis of matrix-metalloproteinase (MMPs)}

The Ae. aegypti (strain: Liverpool) genome in VectorBase (assembly: AaegL3) was analyzed to identify putative MMPs based on the conserved amino acid motif of the MMP catalytic domain: HEXGHXXGXXH where X can be any amio acid residue. We used the National Center for Biotechnology Information ( $\mathrm{NCBI}$ ) conserved protein domain prediction tool to identify the functional domains for the putative AeMMPs. Phylogenetic analyses were conducted using the LINUX-based T-COFFEE program package (181) for the global MMP alignments or MEGA v. 5 (Tempe, AZ, USA) for the AeMMP/Ae. albopictus (Aa) MMP alignments. Prior to alignments, the DNA sequences encoding the variable linker motifs between catalytic and hemopexin domains were removed from all MMP sequences. Global MMP alignments were analyzed using PHYML (maximum likelihood) under the Hasegawa-Kishino-Yano model $(182,183)$ choosing the Caernorhabditis elegans MMP as an outcross group. The AeMMP/AaMMP alignments were further analyzed using MEGA v. 5 using Drosophila MMP1 as an outcross group. Bootstrap values of 1000 were applied in both analyzes. 


\section{5' RACE (Rapid Amplification of cDNA Ends)}

Whole bodies of mosquitoes were collected for total RNA extraction using TRIzol reagent. Total RNA was treated with DNase I to eliminate DNA contamination. Reverse gene-specific primers (Table 1) were designed to anneal 450 base pairs downstream of the known/anticipated 5' end of the RNA transcript. The 5' RACE System for Rapid Amplification of cDNA Ends, version 2.0 (Invitrogen) was used to perform the analysis based on the protocol provided. Resulting PCR products were cloned into TOPO-TA pCR4.0 plasmid vector (Invitrogen) for Sanger sequencing.

\section{cDNA synthesis from mosquito mRNA and SYBR green-based quantitative} (q)PCR

First-strand cDNA was synthesized from $1 \mu \mathrm{g}$ total RNA using a QuantiTect Reverse Transcription Kit (Qiagen, Hilden, Germany) including the random (reverse) primer supplied with the kit. cDNA was diluted 10-fold to be used in QPCR assays. Gene-specific primers were used for QPCR amplification of Aemmps (Table 1). The final reaction volume was $25 \mu$ using a QuantiFast SYBR green PCR kit (Qiagen). qRT-PCR amplification and analysis were carried out using an Applied Biosystems (ABI) 7300 Real-Time PCR System (Applied Biosystems, Foster City, CA, USA). The PCR program was: hold at $95^{\circ} \mathrm{C}$ for 10 min and then at $95^{\circ} \mathrm{C}$ for $15 \mathrm{~s}$ and $60^{\circ} \mathrm{C}$ for $1 \mathrm{~min}$, repeating 40 cycles. The relative abundance of Aemmp transcripts was normalized to that of ribosomal protein S7 (AAEL009496) transcripts as an endogenous reference following the 
Table 1. Primers used for expression profiling of AeMMP genes by qRT-PCR, for 5' RACE of the Aemmp2 transcript, and for recombinant ( $r$ ) protein expression of AeMMP1 and AeMMP2.

\begin{tabular}{|c|c|c|}
\hline Gene & Forward sequence $\left(5^{\prime} \rightarrow 3^{\prime}\right)$ & Reverse sequence $\left(5^{\prime} \rightarrow 3^{\prime}\right)$ \\
\hline Aemmp1 & CAAACAACGTCACAAGCAGAGC & AGCTCTGGAACTCCATGATTGC \\
\hline Aemmp2 & CAACTCGCAACATTTGGAACC & CGTCTTCGTCGAATCTCCAATAA \\
\hline Aemmp4 & ACCAGTGGAGCACAGATTGGAG & CGAGTCCTTTCAGCAGTGTCTCAG \\
\hline Aemmp6 & CAGAAGTTATCCTGCGTGATITG & CGTTACCGGGATGTCGTTAT \\
\hline rpS7 & GGAGAAGAAGTTCTCCGGCAAG & TGAAGGTGTCGACCTTGTGTTC \\
\hline GSP1-Aemmp2 & & TCTGGATTGCGTCCATATGT \\
\hline GSP2-Aemmp2 & & GATATCCGCTTCGCTGCTGTA \\
\hline $\begin{array}{l}\text { GSP-Aemmp2 } \\
\text { (nested) }\end{array}$ & & AGTAACCGTGCAATCGCCAAG \\
\hline rAemmp1 & TACTCAGAATTCATGCACCGAAGTAGGATACATTGG & TACTCACTCGAGGGCTGATTGGAATTTTAGTA \\
\hline rAemmp2 & TACTCAGGATCCATGACCGATCTAGATGCGGGA & TACTCAAAGCTTTCACCACCTATTTCGAAGGTTC \\
\hline
\end{tabular}


$2^{-\Delta \Lambda} C_{T}$ method (184). Samples were tested as three independent biological replicates.

\section{Expression of recombinant AeMMP1 and AeMMP2 proteins}

Full-length Aemmp1 and Aemmp2 cDNAs were amplified from total RNA of Ae. aegypti by RT-PCR. PCR amplicons of Aemmp1 and Aemmp2 cDNAs were inserted into the pET32a expression plasmid vector (Novagen, Madison, WI, USA) using Xhol/ EcoRI and Xhol/BamHI restriction sites, respectively, to express the MMPs along with the N-terminal Trx-, S- and His-tags in E. coli BL21 (DE3) cells (New England Biolabs, Ipswich, MA, USA). Protein production was optimal when bacterial cultures were grown at $37^{\circ} \mathrm{C}$ and shaken at $225 \mathrm{rpm}$. A 5$\mathrm{ml}$ bacterial 'starter' culture from a single colony was grown overnight in Luria-

Bertani (LB) medium (BD Biosciences, San Jose, CA, USA) containing $100 \mu \mathrm{g} / \mathrm{ml}$ ampicillin. The 'starter' culture was then transferred into $500 \mathrm{ml}$ fresh LB medium and incubated for $2-3 \mathrm{~h}$ until the culture reached an optical density $(\mathrm{OD})=0.6$ (at $600 \mathrm{~nm}$ wave-length). Isopropyl $\beta$-D-1-thiogalactopyranoside (IPTG) was then added at a final concentration of $1 \mathrm{mM}$ and the bacterial culture incubated for another $4 \mathrm{~h}$ at $37^{\circ} \mathrm{C}$ and $225 \mathrm{rpm}$.

Generation of AeMMP1 and AeMMP2-specific antibodies and their use in Western blot analysis

Polyclonal antibodies specific to AeMMP1 (pAb-mmp1) and AeMMP2 (pAb-mmp2) were produced by GenScript (Piscataway, NJ, USA) based on the 
peptide amino acid sequences CGTDERPAGDDYNRG and CFEGDSQNRPPVEID (with the N-terminal C being nonspecific), respectively. Both amino acid peptides were derived from the hemopexin domain of the MMPs. To prepare samples, five midguts were dissected from mosquitoes at 24 , 48 and $72 \mathrm{~h}$ post-bloodmeal $(\mathrm{pbm}) /$ post infection with CHIKV and homogenized in $2 \times$ Laemmli sample buffer, boiled for 5 min and centrifuged at $7000 \times g$ for 10 min. For recombinant AeMMP detection, cultures were grown as described above. $2 \mathrm{ml}$ of the induced culture was centrifuged for $5 \mathrm{~min}$ at $11000 \times \mathrm{g}$, to pellet the bacteria. Bacteria pellets were then resuspended in $2 x$ Laemmli sample buffer, boiled for $5 \mathrm{~min}$ and centrifuged at $7000 \times \mathrm{g}$ for $10 \mathrm{~min}$.

Proteins from supernatants were electrophoretically separated by $12 \%$ Sodium-dodecyl sulfate poly-acrylamide gel electrophoresis (SDS-PAGE) and transferred to a nitrocellulose membrane, which was blocked for $1 \mathrm{~h}$ in a $5 \%$ nonfat dried milk/Tris-buffered saline solution (50 mM Tris- $\mathrm{HCl}, 150 \mathrm{mM} \mathrm{NaCl}, 1$ mM ethylenediaminetetraacetic acid (EDTA), 0.1\% Tween 20, pH 7.5; TBST). The membrane was then incubated overnight at $4^{\circ} \mathrm{C}$ in the blocking solution containing either pAb-mmp1 at 1:3000 dilution or pAb-mmp2 at a 1:6000 dilution in TBST. Following overnight incubation with the primary antibody, the membrane was washed three times (10 $\mathrm{min} /$ wash) in TBST before being incubated with anti-rabbit IgG-horse reddish peroxidase (HRP) (Cell Signaling Technology, Danvers, MA, USA), at a dilution of 1:4000 for $2 \mathrm{~h}$ at RT. Finally, the membrane was treated for 1 min with SuperSignal West Pico chemiluminescent substrate (Pierce, Waltham, MA, USA). The immunoreactive 
proteins were visualized by exposing the membrane to an $\mathbf{x}$-ray film. Anti- $\beta$-actinperoxidase antibody (Sigma-Aldrich, St Louis, MO, USA) was used to detect actin as a loading control in each lane.

\section{R-MMP activity assays and rTIMP inhibition}

Recombinant AeMMP1 was activated via incubation with 1 mM APMA or with reaction buffer ( $50 \mathrm{mM}$ Tris- $\mathrm{HCl}, 150 \mathrm{mM} \mathrm{NaCl}, 5 \mathrm{mM} \mathrm{CaCl}_{2}, \mathrm{pH} 7.6$ ) at $37^{\circ} \mathrm{C}$ for $1 \mathrm{~h}$. Twenty ng of activated rAeMMP 1 was then incubated with $20 \mathrm{ng}$ of HuTIMP3 (Anaspec, Fremont, CA), GM6001 (Millipore), or EDTA (Sigma-Aldrich) for $1 \mathrm{~h}$ at room temperature. FS-6 (Millipore) was added to a final concentration of $10 \mu \mathrm{M}$ to the reaction buffer. Fluorescence intensities were measured at Ex $=$ $328 \mathrm{~nm}$ and $\mathrm{Em}=393 \mathrm{~nm}$ with a Perkin-Elmer LS50B spectrometer. Each sample consisted of three independent biological replicates.

\section{Immunofluorescence assays}

Midguts were dissected from sugarfed or CHIKV-infected HWE females at $96 \mathrm{~h}$ post-infection. A group of 10 midguts was fixed in $200 \mu \mathrm{l} \% \mathrm{p}$-formaldehyde (Sigma-Aldrich) for up to 1 week at $4^{\circ} \mathrm{C}$. Midguts were washed three times with phosphate-buffered saline (PBS), and permeabilized with $200 \mu \mathrm{l}$ PBT (1x PBS, $1 \%$ bovine serum albumin, $0.2 \%$ Triton $\mathrm{X}-100$ ) for $1 \mathrm{~h}$ on a rocker at room temperature. Samples of sugarfed mosquitoes were incubated overnight at $4^{\circ} \mathrm{C}$ in $100 \mu \mathrm{l}$ pAb-mmp1 or pAb-mmp2 at a dilution of 1:100 in PBT. Samples were washed four times with $200 \mu \mathrm{l}$ PBST (1x PBS, 0.1\% Triton X-100) and incubated 
at $37^{\circ} \mathrm{C}$ in the dark for $1.5 \mathrm{~h}$ with $100 \mu \mathrm{l}$ anti-rabbit lgG Alexa Fluor 594 (Cell Signaling Technology) diluted 1:200 in PBT. Samples of CHIKV-infected mosquitoes were incubated overnight at $4^{\circ} \mathrm{C}$ with a mixture of anti-CHIKV monoclonal antibody [B1414] (Abcam, Cambridge, UK), diluted 1:100 and pAbmmp1 or pAb-mmp2 at a dilution of 1:100 in PBT. Samples were washed four times with $200 \mu \mathrm{l}$ PBST and incubated at $37^{\circ} \mathrm{C}$ in the dark for $1.5 \mathrm{~h}$ with $100 \mu \mathrm{l}$ anti-mouse IgG Alexa Fluor 488 (Cell Signaling Technology), diluted 1:800 and anti-rabbit IgG Alexa Fluor 594 (Cell Signaling Technology) diluted 1:200 in PBT. To stain nuclei, $5 \mu \mathrm{l}$ of a $0.5 \mu \mathrm{g} / \mu \mathrm{l}$ DAPI (Invitrogen) was added to each tube followed by incubation at RT for $30 \mathrm{~min}$. Actin filaments of midguts not infected with CHIKV were stained with $200 \mu \mathrm{l}$ of a $1 \mathrm{unit} / \mathrm{ml}$ solution of Alexa Fluor Phalloidin 488 (Invitrogen) for another 30 min at room temperature. After four washes with PBST, midguts were placed individually on glass slides, and mounted with Fluoromount-G (Electron Microscopy Sciences, Hatfield, PA, USA). Samples were viewed and analyzed under an inverted spectral confocal microscope (TCP SP8 MP, Leica Microsystems, Wetzlar, Germany).

\section{Midgut sample preparation for SEM}

Unless otherwise stated, all reagents were purchased from Electron Microscopy Sciences (Hatfield, PA, USA) and all specimen preparation was performed at the Electron Microscopy Core Facility, University of Missouri. Midguts were dissected from HWE mosquitoes and fixed in $2 \%(\mathrm{v} / \mathrm{v})$ paraformaldehyde, $2 \%(\mathrm{v} / \mathrm{v})$ glutaraldehyde containing $100 \mathrm{mM}$ sodium 
cacodylate buffer, $\mathrm{pH} 7.35$. Fixed tissues were rinsed with $100 \mathrm{mM}$ sodium cacodylate buffer, $\mathrm{pH} 7.35$ containing $130 \mathrm{mM}$ sucrose and further rinsed with water. Secondary fixation was performed using 1\% osmium tetroxide (Ted Pella, Redding, CA, USA) in cacodylate buffer followed by treatment in a Pelco Biowave (Ted Pella) operated at 100 Watts for 1 min. Specimens were next incubated at $4^{\circ} \mathrm{C}$ for $1 \mathrm{~h}$, then rinsed with cacodylate buffer and thereafter with distilled water. Using the Pelco Biowave, a graded dehydration series (100 Watts for 40 seconds (s) per exchange) was performed using ethanol. Samples were dried using the Tousimis Autosamdri 815 critical point dryer (Tousimis, Rockville, MD, USA), and then sputter-coated with $10 \mathrm{~nm}$ of platinum using the EMS 150TES Sputter Coater. Images were acquired with a FEI Quanta 600F environmental scanning electron microscope (FEI, Hillsboro, OR, USA).

\section{Midgut sample preparation for TEM}

Mosquito midguts were dissected and fixed in $100 \mathrm{mM}$ sodium cacodylate buffer, $\mathrm{pH} 7.35$ supplemented with $2 \%(\mathrm{v} / \mathrm{v})$ paraformaldehyde and $2 \%(\mathrm{v} / \mathrm{v})$ glutaraldehyde. Each midgut sample was oriented and suspended in HistoGel (Thermo Scientific, Kalamazoo, MI, USA). Next, samples were rinsed with 100 mM sodium cacodylate buffer, pH 7.35 (Sigma Aldrich, St. Louis, MO, USA) containing $130 \mathrm{mM}$ sucrose. Secondary fixation of the sample was conducted in a Pelco Biowave operated at 100 Watts for 1 min using a $100 \mathrm{mM}$ sodium cacodylate buffer supplemented with $1 \%$ osmium tetroxide. Specimens were incubated at $4^{\circ} \mathrm{C}$ for $1 \mathrm{~h}$, then rinsed with cacodylate buffer followed by distilled 
water. En bloc staining was performed using $1 \%$ aqueous uranyle acetate and incubation at $4^{\circ} \mathrm{C}$ overnight followed by rinsing with distilled water. Using the Pelco Biowave, a graded dehydration series (100 Watts for $40 \mathrm{~s}$ per exchange) was performed in which ethanol was initially used followed by transition to acetone before dehydrated specimens were finally infiltrated with Epon resin (250 Watt for $3 \mathrm{~min}$ ) and polymerized at $60^{\circ} \mathrm{C}$ overnight. Sample sections were cut to a thickness of $85 \mathrm{~nm}$ using an ultra-microtome (Ultracut UCT, EM UC7, Leica Microsystems, Wetzlar, Germany) equipped with a diamond knife (Diatome, Hatfield, PA, USA). Images were acquired with a JEOL JEM 1400 transmission electron microscope (JEOL, Peabody, MA, USA) at $80 \mathrm{kV}$ using a Gatan Ultrascan 1000 CCD camera (Gatan, Pleasanton, CA, USA).

\section{Analyzing chikungunya (CHIK) virion density in midgut epithelial cells and measuring midgut $B L$ thickness in TEM images}

In each TEM micrograph, CHIK virions were counted and the surface area of the tissue containing virions was determined based upon the pixel size of each image using the software Gatan Microscopy Suite (Gatan). At least 18 TEM micrographs from three different midgut preparations were analyzed per time point. Thickness of the intact midgut $B L$ was measured in several locations of three different midguts per time point. Measurements were conducted in zones absent of muscle tissue where the $B L$ was often spliced and disorganized. $B L$ measurements were based on the pixel size of each image as analyzed with the Gatan Microscopy Suite. 


\section{Midgut sample preparation for serial block face imaging via FIB-SEM}

Midgut samples were prepared following a modified version of the NCMIR protocols for three-dimensional EM with changes to the sample mounting procedure $(185,186)$. Primary midgut sample fixation was carried out as described above for SEM and TEM sample preparations. Secondary sample fixation was performed using equal parts $4 \%(w / v)$ osmium tetroxide (Ted Pella) and $3 \%(\mathrm{w} / \mathrm{v})$ potassium ferrocyanide in $100 \mathrm{mM}$ cacodylate buffer, $\mathrm{pH} 7.35$ followed by treatment in a Pelco Biowave operated at 100 Watts for 1 min. Specimens were incubated on ice for $1 \mathrm{~h}$, then first rinsed with cacodylate buffer and then with distilled water. Samples were then incubated at room temperature for $1 \mathrm{~h}$ in a $1 \%(\mathrm{w} / \mathrm{v})$ thiocarbohydrazide solution followed by distilled water rinses. Rinsed tissues were incubated in an additional $2 \%(\mathrm{w} / \mathrm{v})$ aqueous osmium tetroxide solution for 30 min at RT, then rinsed again with distilled water. Initial en bloc staining was performed using $1 \%(\mathrm{w} / \mathrm{v})$ aqueous uranyl acetate and incubation at $4^{\circ} \mathrm{C}$ overnight followed by a finale rinse with distilled water. Additional en bloc staining was performed using Walton's lead aspartate solution for $30 \mathrm{~min}$ at $60^{\circ} \mathrm{C}$ followed by additional distilled water rinses. Using the Pelco Biowave, a graded dehydration series (per exchange, 100 Watts for $40 \mathrm{~s}$ ) was performed using ethanol followed by transitioning into acetone. Dehydrated tissues were then infiltrated with Durcupan ACM resin (250 Watt for 3 min) and polymerized at $60^{\circ} \mathrm{C}$ overnight. All samples were trimmed and sectioned to a thickness of $85 \mathrm{~nm}$ to be initially examined by TEM (JEOL JEM 1400 operated at 
$80 \mathrm{kV})$. This allowed for precise identification of virus-infected regions in the tissue and measurements were taken using landmarks that could be easily identified in the FIB-SEM. To ensure all FIB-SEM data were collected in a cross section, the tissue was mounted with the block face perpendicular to the stub surface. The area of interest was identified using the landmarks established in TEM and a $2 \mu \mathrm{m}$ layer of platinum was deposited on the block face using the ion column. Next, the area of interest was polished using an ion beam setting of 30 $\mathrm{kV}, 1 \mathrm{nA}$ and $20 \mathrm{~nm}$ sections were subsequently removed from the sample using ion beam settings of $30 \mathrm{kV}, 1 \mathrm{nA}$. FIB-SEM data was collected using a FEI Scios Analytical Dualbeam SEM operated at $2 \mathrm{kV}$ with $0.2 \mathrm{nA}$ current and equipped with a T1-BSE detector to capture the sample images. Using the Amira software suite v. 5.3.1 (FEI Visualization Science Group, Hillsboro, OR, USA), the recorded stack of 108 images from the sample was aligned and segmented to render the three-dimensional model. Based on the data obtained with the Amira software, the model was then graphically refined in Maxon Cinema 4D R19 (MAXON Computer GmbH, Friedrichsdorf, Germany) using the software OTOY Octane Render V3 (OTOY Inc., Los Angeles, CA, USA).

\section{Generation of cDNA templates from viral RNA}

Mosquitoes orally infected with CHIKV or MAYV were dissected to separate midguts from their carcasses at $8,24,32,48,72$, and $96 \mathrm{~h}$ pibm. Total RNA was extracted from mosquito midguts (10 midguts per pool) or carcasses (three carcasses per pool) using TRIzol reagent. Total RNA $(1 \mu \mathrm{g})$ was reverse 
transcribed using the SuperScript III First-Strand Synthesis System (Life Technologies). The reaction components and thermal conditions were the following: $1 \mu \mathrm{g}$ RNA was mixed with $2 \mu \mathrm{l}$ of virus- and strand specific primer (10 $\mathrm{pmol} / \mu \mathrm{l}$; Table 2) and $2 \mu \mathrm{l}$ of dNTP $(10 \mathrm{mM})$ before being incubated at $65^{\circ} \mathrm{C}$ for 5 min and snap cooled on ice. The heat denatured RNA was mixed with $4 \mu \mathrm{l}$ of $5 x$ reverse transcriptase (RT) buffer, $1 \mu \mathrm{l}$ of $0.1 \mathrm{M}$ dithiothreitol (DTT), $1 \mu \mathrm{l}$ of reverse transcriptase $(15 \mathrm{U} / \mu \mathrm{l})$, and nuclease-free water to a final volume of $20 \mu \mathrm{l}$. The reaction mixture was incubated at $55^{\circ} \mathrm{C}$ for $60 \mathrm{~min}$ and inactivated at $85^{\circ} \mathrm{C}$ for 5 $\min$.

Taqman qPCR assays for the virus-specific detection of plus- and minusstrand RNAs

Probes were custom-designed by Integrated DNA Technologies (Coralville, IA, USA) to detect viral plus- and minus-strand RNAs via Taqman qPCR. The Taqman probes contained a 5' FAM reporter and two quenchers, ZEN and IABkFQ, located in the center and at the $3^{\prime}$ end of the molecule, respectively. The ZEN quencher aided in reducing background, and sensitivity of the probe. The oligonucleotide primers contained 5' AT-rich flaps (5'AATAAATCATAA3') to increase detection specificity (Table 2). The qPCR amplification was performed using the iTaq Universal Probes Supermix (Bio-Rad, Hercules, CA, USA). Each reaction had a total volume of $20 \mu \mathrm{l}$ consisting of $10 \mu \mathrm{l}$ 
Table 2. Oligonucleotide primers used for Taqman qRT-PCR to detect CHIKV and MAYV cDNAs. Primers used for qPCR detection have 5' at rich flaps not specific to virus sequence.

\begin{tabular}{|c|c|}
\hline Primers for RT & $5^{\prime} \rightarrow 3^{\prime}$ \\
\hline CHIKV + strand & TAATACGACTCACTATAGGGACGAAACCACTGTATCACAGCG \\
\hline CHIKV - strand & TCGGCAGTATCGTGAATTCGATGCAGACCTGACGGAAGGTAGACG \\
\hline MAYV + strand & TAATACGACTCACTATAGGGCGGTTTCATTCTCTTCTTCCTC \\
\hline MAYV - strand & TCGGCAGTATCGTGAATTCGATGCCCAGGCAATGAAAGGAGTACG \\
\hline \multicolumn{2}{|l|}{ Primers for qPCR } \\
\hline CHIKV + F & aataaatcataaCGTACTGTTCTCAGTCGGGTC \\
\hline CHIKV + R & aataaatcataaTAATACGACTCACTATAGGG \\
\hline CHIKV - F & aataaatcataaTCGGCAGTATCGTGAATTCGATGC \\
\hline CHIKV -R & aataaatcataaGATGGAACACTGAAGGTAAGTGC \\
\hline MAYV + F & aataaatcataa CCTACCCAACATATGCAACCA \\
\hline MAYV + R & aataaatcataaTAATACGACTCACTATAGGG \\
\hline MAYV - F & aataaatcataaTCGGCAGTATCGTGAATTCGATGC \\
\hline MAYV - R & aataaatcataaAAGGTGTCCCTCAGTCAGT \\
\hline \multicolumn{2}{|l|}{ Probes } \\
\hline CHIKV & TACCCGGAGAGCCGTAAGCTTCTTA \\
\hline MAYV & ACGAACAGGTGTTGAAAGCCAGGA \\
\hline
\end{tabular}


of $2 x$ master mix, forward and reverse primers at a concentration of $900 \mathrm{nM}, 2 \mu \mathrm{l}$ of cDNA template, and the Taqman probe at a concentration of $250 \mathrm{nM}$.

Amplification reactions were performed for 40 cycles under the following thermocycling conditions: Initial denaturation at $95^{\circ} \mathrm{C}$ for $2 \mathrm{~min}$, annealing at $60^{\circ} \mathrm{C}$ for 30 $\mathrm{s}$, and extension at $60^{\circ} \mathrm{C}$ for $30 \mathrm{~s}$. The results were analyzed using standard curves, which were generated for the detection of CHIKV and MAYV viral RNAs by cloning cDNA segments of each virus containing the sequences of the $\mathrm{PPCR}$ primers and the probe into the pCR-2.1-TOPO TA plasmid vector (Invitrogen). DNA plasmid copy numbers were calculated based on their lengths and concentrations. For each qPCR assay, a new 10 -fold dilution series of the standard curve was generated. Each sample consisted of three independent biological replicates.

\section{Saliva collection from individual mosquitoes}

Saliva was collected from individual MAYV challenged Ae. aegypti mosquitoes at 2, 4, 6, 7, and 12 days pibm using a forced salivation method. The proboscis of each female mosquito, whose wings and legs had been clipped off, was inserted into a $1 \mathrm{~mm}$ glass capillary tube filled with 3-5 $\mu$ l Cargille Type $B$ immersion oil (Cargille Laboratories, Cedar Grove, NJ, USA). After 40 min, saliva was collected from those capillaries in which droplets of saliva exuding from the proboscis were visible. Saliva samples were recovered from each capillary tube by centrifugation at $3,000 \mathrm{~g}$ for $5 \mathrm{~min}$ in a $1.5 \mathrm{ml}$ Eppendorf tube containing 200 $\mu \mathrm{l}$ sample processing buffer. After addition of another $200 \mu \mathrm{l}$ of sample 
processing buffer, each sample was vortexed and sterile-filtered using a $0.2 \mu \mathrm{m}$ syringe filter (Pall Life Sciences). Virus from saliva samples was amplified in Vero cells seeded onto 24 -well plates at $\sim 90 \%$ confluency. Cell growth medium was removed before cells in each well were inoculated with $180 \mu$ saliva samples for

$1 \mathrm{~h}$ at $37^{\circ} \mathrm{C}$. Thereafter, $1 \mathrm{ml}$ of DMEM supplemented with $7 \% \mathrm{FBS}$ was added to each well. Cells were observed daily for the development of cytopathic effects (CPE) until 7 days pibm.

Saliva samples of 10-day old Ae. albopictus CoMO females were collected at 3 days post-intrathoracic injection of $210 \mathrm{nl}(=700 \mathrm{pfu} / \mathrm{ml})$ of MAYV TRVL 4675 (passage 1 virus stock, titer: $6.0 \times 10^{6} \mathrm{pfu} / \mathrm{ml}$ ) per female using the Nanoject II injection system (Drummond Scientific, Broomall, PA, USA). Saliva samples were processed and tested via amplification in Vero cells as described above. CPE in Vero cells was recorded at 7 days post-inoculation. Head tissues of individual females were collected and processed as described above to assess virus titers in plaque assays.

\section{Sequencing of viral genomes and phylogenetic analysis}

For both MAYV IQT 4235 and MAYV TRVL 4675, supernatant was harvested at 30-39 h post infection based on $>50 \%$ CPE. Viral RNA was isolated from $280 \mu \mathrm{l}$ of sample using the QIAamp Viral RNA Mini Kit (Qiagen). cDNA synthesis was performed using $1 \mu \mathrm{g}$ of total RNA and the Superscript III FirstStrand Synthesis System (Invitrogen) in combination with an oligo (dT) 20 primer. Genome segments were amplified using AccuPrime Taq DNA Polymerase High 
Fidelity (Invitrogen) and primer pairs designed to walk through the genome of each MAYV strain. PCR products were purified and Sanger sequenced at the DNA Core of the University of Missouri. 3' RACE was performed with the 3' RACE System for Rapid Amplification of DNA Ends kit (Invitrogen). Sequences were then assembled and aligned using the DNASTAR LaserGene Molecular Biology suite software (version 15). Phylogenetic analyses were conducted by aligning the full-length nucleotide sequences of 26 MAYV strains and comparing them using the Maximum Likelihood method based on the General Time Reversible model. Bootstrap values of 1000 were applied to analysis performed in MEGA X.

\section{qRT-PCR for virus-specific detection in samples containing mixed infections}

HWE females were exposed to bloodmeals that contained either MAYVIQT 4675 (titer: $3.3 \times 10^{6} \mathrm{pfu} / \mathrm{ml}$ ), CHIKV 37997 (titer: $2.7 \times 10^{6} \mathrm{pfu} / \mathrm{ml}$ ) or both viruses in combination. Another group of HWE mosquitoes received an initial bloodmeal containing either MAYV IQT 4675 or CHIKV 37997 followed by a subsequent bloodmeal at 6 days pibm containing the virus (either MAYV or CHIKV), which had not been initially acquired. Controls consisted of mosquito groups that were initially fed with bloodmeals consisting of either MAYV or CHIKV followed by non-infectious bloodmeals (a mixture of non-infected cell culture supernatant and defibrinated sheep blood at a 1:1 ratio) at 6 days pibm. Saliva samples of mosquitoes of both experimental groups were collected at 12 
days pibm as described above except that $50 \mu \mathrm{l}$ of saliva samples from five individuals were pooled together and then diluted in $750 \mu$ IRIzol LS (Life Technologies) for total RNA extraction according to the manufacturer's instructions. cDNA synthesis was performed with $500 \mathrm{ng}$ of extracted total RNA using the Superscript III First-Strand Synthesis System (Invitrogen) and CHIKV and MAYV specific primers (Table 2). The multiplex qPCR reaction was performed using the iTaq Universal Probes Supermix (Bio-Rad) with a total reaction volume being $20 \mu \mathrm{l}$. Each reaction contained $2 \mu \mathrm{l}$ of cDNA template, virus specific primers for both CHIKV and MAYV at a final concentration of 900 $\mathrm{nM}$, and a virus-specific probe for both CHIKV and MAYV at a final concentration of $250 \mathrm{nM}$ (Table 2). qRT-PCR reactions were conducted using a StepOnePlus Real-Time PCR system (Applied Biosystems, Foster City, CA, USA). The 40cycle qRT-PCR program was as follows: denaturing at $95^{\circ} \mathrm{C}$ for $15 \mathrm{~s}$, followed by a 1 min annealing and extension step at $60^{\circ} \mathrm{C}$. Probes specific for either $\mathrm{CHIKV}$ or MAYV contained two distinct reporters, 5' FAM or 5'JOE respectively, and two quenchers, ZEN located in the center of the probe and IABkFQ at its $3^{\prime}$ end. qRTPCR results were analyzed based on standard curves that were generated by cloning a CHIKV or MAYV derived cDNA segment into the pCR-2.1-TOPO TA plasmid vector (Invitrogen). DNA plasmid copy numbers were calculated based on plasmid sizes and concentrations. Based on threshold cycle (Ct) values of the negative controls, $\mathrm{Ct}$ values that measured above 36.3 were deemed undetectable. Consequently, samples with Ct values $>36.3$ were considered 
negative for viral RNA. All samples consisted of three independent biological replicates.

\section{Statistical analyses}

Statistical analyses were performed using the GraphPad Prism (version 5) software suite. Median virus titers were analyzed using the Mann-Whitney U-test followed by the Kruskal-Wallis test. Infection rates were compared using Fisher's Exact test. BL thickness and CHIK virion densities were analyzed by One-way ANOVA across timepoints, followed by Tukey's multiple comparison test. In the qRT-PCR experiments, data analysis was conducted using one-way analysis of variance (ANOVA) followed by Tukey's multiple comparisons test or using Dunn's multiple comparison test to identify means that were significantly different from others. All tests were considered significant at $p \leq 0.05$. 


\section{CHAPTER III}

\section{Identification and Initial Characterization of Matrix Metalloproteinases in the Yellow Fever Mosquito, Aedes aegypti}

This chapter has been published in the journal, Insect Molecular Biology

Asher M. Kantor, ${ }^{1}$ Shengzhang Dong, ${ }^{1}$ Nicole L. Held, ${ }^{1}$ Egide Ishimwe,${ }^{2}$ A. Lorena Passarelli, ${ }^{2}$ Rollie J. Clem, ${ }^{2}$ and Alexander W.E. Franz ${ }^{1}$. "Identification and Initial Characterization of Matrix Metalloproteinases in the Yellow Fever Mosquito, Aedes Aegypti." Insect molecular biology 26.1 (2017): 113-126.

AMK, NLH, AJP, RJC and AWEF conceived and designed the experiments; AMK, SD, and El performed the experiments; AMK, and SD analyzed the data; AMK and AWEF wrote and edited the paper.

${ }^{1}$ Department of Veterinary Pathobiology, University of Missouri, Columbia, Missouri, United States of America

${ }^{2}$ Division of Biology, Kansas State University, Manhattan, Kansas, United States of America 


\section{Abstract}

Aedes aegypti is a major vector for arboviruses such as dengue, chikungunya and Zika viruses. During acquisition of a viremic bloodmeal, an arbovirus infects mosquito midgut cells before disseminating to secondary tissues, including the salivary glands. Once virus is released into the salivary ducts it can be transmitted to another vertebrate host. The midgut is surrounded by a basal lamina $(\mathrm{BL})$ in the extracellular matrix, consisting of a proteinaceous mesh com- posed of collagen IV and laminin. BL pore size exclusion limit prevents virions from passing through. Thus, the BL probably requires remodeling via enzymatic activity to enable efficient virus dissemination. Matrix metalloproteinases (MMPs) are extracellular endopeptidases that are involved in remodeling of the extracellular matrix. Here, we describe and characterize the nine Ae. aegypti encoded MMPs, AeMMPs 1-9, which share common features with other invertebrate and vertebrate MMPs. Expression profiling in Ae. aegypti revealed that Aemmp4 and Aemmp6 were upregulated during metamorphosis, whereas expression of Aemmp1 and Aemmp2 increased during bloodmeal digestion. Aemmp1 expression was also upregulated in the presence of a bloodmeal containing chikungunya virus. Using polyclonal antibodies, AeMMP1 and AeMMP2 were specifically detected in tissues associated with the mosquito midgut. 


\section{Introduction}

The yellow fever mosquito, Aedes aegypti, is a major vector for flaviviruses (family: Flaviviridae; genus: Flavivirus) such as dengue and Zika viruses, and alphaviruses (family: Togaviridae; genus: Alphavirus) such as chikungunya virus (CHIKV). These viruses are currently the most prevalent arboviruses that infect humans and are cocirculating in many regions of the tropical world $(187,188)$.

The arboviral disease cycle requires persistent infection of an arthropod vector before virus can be transmitted to a new vertebrate host (189). A mosquito acquires virus from a viremic host along with the bloodmeal. The bloodmeal enters the midgut and within several hours, virions will infect the epithelial cells lining the midgut. Hours later, virions disseminate from midgut epithelial cells to infect secondary tissues or cells, such as hemocytes, fat body, nerve tissue and eventually the salivary glands. Once these are infected, virus is released along with saliva when the mosquito is feeding on a vertebrate host. Like all epithelia, the midgut epithelium is lined with a basal lamina $(\mathrm{BL})$, a sheet-like network of extracellular matrix (ECM) components including collagen IV, laminin and proteoglycans (116). The typical BL pore size exclusion limit in mosquitoes is only $10 \mathrm{~nm}$, which is too small for flavivirus or alphavirus virions, which are $50-80$ $\mathrm{nm}$ in diameter, to pass through (121). Thus, it has been postulated that the BL needs to be remodeled, thereby increasing its pore size exclusion limit before virions can disseminate from the midgut to secondary tissues. This hypothesis is supported by observations with another model system, the lepidopteran insect 
Trichoplusia ni and the baculovirus Autographa californica Multicapsid nucleopolyhedrovirus (AcMNPV), which also must overcome a midgut escape barrier in the caterpillar in order to establish a systemic infection $(190,191)$. A midgut escape mechanism for AcMNPV has been proposed, which involves the activity of a viral fibroblast growth factor, matrix metalloproteinases (MMPs) and caspases, resulting in $\mathrm{BL}$ remodeling to enhance virus dissemination from the midgut.

MMPs are expressed as zymogens with a characteristic domain structure consisting of a propeptide domain, a catalytic domain and a hemopexin domain (192). The propeptide domain is located at the $\mathrm{N}$ - terminus and contains a conserved cysteine residue within the amino acid (aa) sequence motif, PRCGXXD, known as the cysteine switch (CS), which helps to maintain an inactive MMP conformation. Upon activation of the enzyme, the propeptide domain is cleaved releasing the cysteine from a $\mathrm{Zn}^{2+}$ ion, allowing access to the catalytic domain (193). The catalytic domain is located downstream of the propeptide domain and is identified by the highly conserved amino acid sequence motif HEXGHXXGXXH, containing the Zn- binding site (194). The hemopexin domain is located at the C-terminus of the protein and is connected to the catalytic domain via a flexible linker region (195). The hemopexin domain plays a major role in substrate specificity $(196,197)$. To date, there have been at least 23 human MMPs (HuMMPs) identified and characterized $(128,198,199)$.

Here, we examined nine putative MMPs found in the genome of $A e$. aegypti. We analyzed the phylogenetic relationships of these Ae. aegypti MMPs 
(AeMMPs) with those of other arthropods and the well-described HuMMPs. We used quantitative reverse transcription PCR (qRT-PCR) to conduct expression profiling of Aemmp genes during metamorphosis, bloodmeal digestion and arbovirus infection using CHIKV as a model. Antibodies to two AeMMPs, which are overexpressed during bloodmeal digestion were produced to analyze their expression at the protein level in Western blots and in situ.

\section{Results}

\section{Identification of MMPs in the genome of Ae. aegypti}

Searches of the genome sequence of Ae. aegypti in VectorBase (AaegL3) revealed the presence of nine putative AeMMP genes, which we are designating Aemmp1-9 according to homologies with other arthropod MMPs and the order of their gene accession numbers in VectorBase (Table 3). Aemmp1 and Aemmp2 are orthologues of $m m p 1$ and $m m p 2$ of Ixodes scapularis, Drosophila melanogaster, Bombyx mori, Tribolium castaneum, Anopheles gambiae, Culex quinquefasciatus and Aedes albopictus, respectively. Aemmp3 is an orthologue of mmp3 of T. castaneum, An. gambiae, Cx. quinquefasciatus and Ae. albopictus. With the exception of Aemmp2 (chromosome 3) and Aemmp3 (chromosome 1), all AeMMP genes are encoded on chromosome 2. For Aemmp9, a chromosome location could not be identified. AeMMPs predict similar lengths as HuMMPs (261-707 aa), with AeMMP3 (605 aa) and AeMMP9 (273 aa) having the longest and shortest amino acid sequences, respectively (Table 3). Five of the nine AeMMPs contain the signature propeptide, catalytic and 
hemopexin domains, whereas AeMMP9 is a truncated copy of AeMMP1, lacking the hemopexin domain (Fig. 4A). Generally, the catalytic domains of MMPs are highly conserved, whereas propeptide and hemopexin domains typically show high levels of divergence $(192,197)$. Alignment of the propeptide domains of the AeMMPs showed the presence of a CS motif (Fig. 4B). Even though the CS motifs of AeMMPs vary slightly from the canonical mammalian CS motif, PRCGXXD, they all contain the conserved cysteine residue at or nearby aa position 80. Other MMPs such as HuMMP28 also encode a variation of the canonical CS motif without compromising their function (200). In addition, AeMMP1, AeMMP3, AeMMP6, AeMMP7 and AeMMP9 contain a putative RXXR furin cleavage site. Alignment of the catalytic domains revealed that the MMP hallmark amino acid sequence motif HEXGHXXGXXH was present in all nine AeMMPs (Fig. 4C). All AeMMPs, except AeMMP9, possess a flexible linker region that links the catalytic domain to the C-terminal hemopexin domain. Further, AeMMP1 is the only AeMMP that appears to be membrane-associated as it is predicted to possess a GPI anchor (based on in silico prediction using the web-based tool GPI-Som: http:// genomics.unibe.ch/cgi-bin/gpi.cgi?ref5 1\&id). We also noticed that Aemmp2 had been incorrectly annotated in VectorBase. We performed 5' 


\begin{tabular}{|c|c|c|c|c|c|c|}
\hline Gene ID & Supercontig & Chromosome & Designation & Homology to & $\mathrm{aa}^{\ddagger}$ & Transcript \\
\hline AAEL005666 & 1.169 & $2 p^{*}$ & Aemmp1 $1^{\Uparrow}$ & $A a, A g, B, C, D, I, T^{\dagger}$ & 573 & 3050 \\
\hline AAEL000788 & 1.16 & $3 q$ & Aemmp $2^{\text {ๆ }}$ & $\mathrm{Aa}, \mathrm{Ag}, \mathrm{B}, \mathrm{C}, \mathrm{D}, \mathrm{I}, \mathrm{T}$ & $526^{\S}$ & $1581^{\S}$ \\
\hline AAEL002982 & 1.74 & $1 q$ & Aemmp3 & $\mathrm{Aa}, \mathrm{Ag}, \mathrm{C}, \mathrm{T}$ & 605 & 2214 \\
\hline AAEL002655 & 1.63 & $2 q$ & Aemmp $4^{\text {ๆ }}$ & $\mathrm{Aa}, \mathrm{C}$ & 481 & 2249 \\
\hline AAEL002661 & 1.63 & $2 q$ & Aemmp5 & $\mathrm{Aa}, \mathrm{C}$ & 430 & 1615 \\
\hline AAEL002665 & 1.63 & $2 q$ & Aemmp6 & $\mathrm{Aa}, \mathrm{C}$ & 492 & 1507 \\
\hline AAEL002672 & 1.63 & $2 q$ & Aemmp7 & $\mathrm{Aa}, \mathrm{C}$ & 493 & 2347 \\
\hline AAEL002677 & 1.63 & $2 q$ & Aemmp8 & $\mathrm{Aa}, \mathrm{C}$ & 479 & 1440 \\
\hline AAEL017046 & 1.1354 & ? & Aemmp9 & - & 273 & 822 \\
\hline
\end{tabular}

Table 3. Identification and designation of putative matrix metalloproteinase (MMP) genes in the genome of $A e$. aegypti based on VectorBase searches.

*Based on supercontig mapping data (Juneja et al., 2014; Timoshevsky et al., 2014). †Aa, Aedes albopictus; Ag, Anopheles gambiae; B, Bombyx mori; C, Culex quinquefasciatus; D, Drosophila melanogaster; I, Ixodes scapularis; T, Tribolium castaneum.

faa, amino acids.

§aa sequence determination and transcript length are based on 5' rapid amplification of cDNA ends data shown in Fig. 5

ImRNA transcript was detectable by RT-PCR. 
A

\section{CS}

propeptide catalytic $=$ hemopexin no furin-cleavage site: MMP4

CS RXXR propeptide catalytic

hemopexin

furin-cleavage site: MMP3, MMP6, MMP7

$$
\text { CS RXXR }
$$

$$
\text { propeptide catalytic }
$$

hemopexin

GPI

furin-cleavage site, GPI anchor: MMP1

CS RXXR propepetide catalytic

no hemopexin domain: MMP9

catalytic nemopexin no propeptide domain: MMP5, MMP8

catalytic hemopexin no propeptide domain, long hinge region: MMP2

AeMMP 1, AeMMP 9: HEFGHSLGLSHSDVRSALM AeMMP2: HEFGHSLGLGHSSVKEAIM AeMMP 3: $\quad$ HELGHSLGLAHSPVYSSLM AeMMP 4: HEIGHSLGLEHSNTKGALM AeMMP 5: $\quad$ HEIGHSLGLLHSASRSSIM AEMMP 6: HEIGHSLGLEHSRSKASLM AEMMP 7: HEIGHTLGLDHTVSRTSIM AEMMP 8: $\quad$ HEIGHALGMSHSSSRASIM DMMMP1: $\quad$ HEFGHSLGLSHSDQSSALM HUMMP 9: $\quad$ HEFGHALGLDHSSVPEALM

B

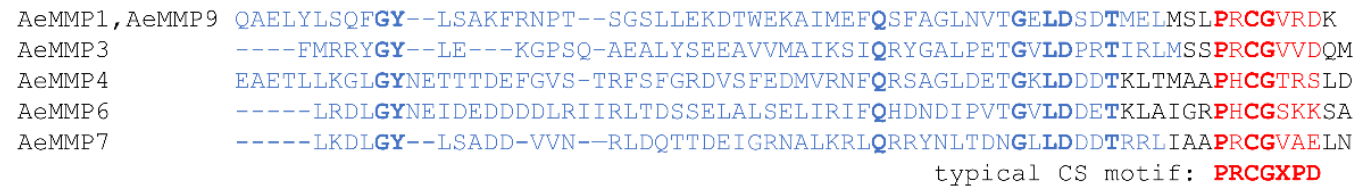

AeMMP1,AeMMP9 QAELYLSQFGY--LSAKFRNPT--SGSLLEKDTWEKAIMEFQSFAGLNVTGELDSDTMELMSLPRCGVRDK AeMMP 3 ---EMRRYGY--LE---KGPSQ-AEALYSEEAVVMAIKSIQRYGALPETGVLDPRTIRLMSSPRCGVVDQM AeMMP 4 EAETLLKGLGYNETTTDEFGVS-TRFSFGRDVSFEDMVRNEQRSAGLDETGKLDDDTKLTMAAPHCGTRSLD AeMMP 6 ----LRDLGYNEIDEDDDDLRIIRLTDSSELALSELIRIFQHDNDIPVTGVLDDETKLAIGRPHCGSKKSA AeMMP7 -----LKDLGY--LSADD-VVN--RLDQTTDEIGRNALKRLQRRYNLTDNGLLDDDTRRLIAAPRCGVAELN typical CS motif: PRCGXPD

Figure 4. Characterization of the functional domains of Aedes aegypti matrix metalloproteinases (AeMMPs).

(A) Mapping of key domains for AeMMPs1-9. (B) Amino acid sequence alignment of the propeptide domains of AeMMPs. Cysteine switch (CS) motifs are highlighted in red; a canonical CS motif from a vertebrate MMP is shown for comparison. In bold, conserved residues. (C) Amino acid sequence alignment of the catalytic domains of AeMMPs. In bold, conserved residues of the zinc-binding motif of the catalytic domain; underlined, conserved methionine outside the zinc binding motif providing a hydrophobic base for zinc ion interaction. Catalytic domain amino acid sequences of Drosophila melanogaster MMP1 (DmMMP1) and human MMP9 (HuMMP9) are included for comparison. Abbreviations: RXXR, furin cleavage site; GPI, glycosylphosphatidylinositol. 
rapid amplification of cDNA ends (5'RACE) for Aemmp2 and discovered that the translational start site is located at the end of exon 4 (Fig. 5). Also, when aligning the amino acid sequence of AeMMP2 with its homologue from Ae. albopictus (AaMMP2), it became obvious that according to its annotation in VectorBase, AeMMP2 but not AaMMP2, contains 120 additional N-terminal amino acids. Regardless, AeMMP2, similar to AeMMP5 and AeMMP8, lacks a propeptide domain. In summary, five of the nine AeMMPs that we have annotated possess the typical domains found in most MMPs (propeptide, catalytic and hemopexin domains), whereas three of them lack the propeptide, and one lacks the hemopexin domain, demonstrating a high level of diversity within this protein family in mosquitoes.

\section{Similarity of AeMMPs with MMPs of humans and other arthropods}

Maximum likelihood phylogenetic analyzes based on nucleotide sequences revealed that arthropods such as D. melanogaster, B. mori, $T$. castaneum, I. scapularis and mosquitoes (An. gambiae, Cx. quinquefasciatus and Ae. albopictus) all possess orthologues of Aemmp1 and Aemmp2 (Fig. 6A). T. castaneum and mosquitoes also share orthologues of Aemmp3. Apparently, the Culex and Aedes genomes underwent a further MMP gene expansion, with Culex having five additional MMPs, three of which are orthologous between Ae. aegypti and Cx. quinquefasciatus, and Aedes spp. possessing up to seven additional MMPs (Fig. 6A, B). All arthropod MMPs cluster together but separately from the mammalian MMPs, with the arthropod 
Chromosome 3q, supercontig1.16: nt position 1,744,815-1,794,347 (reverse strand)

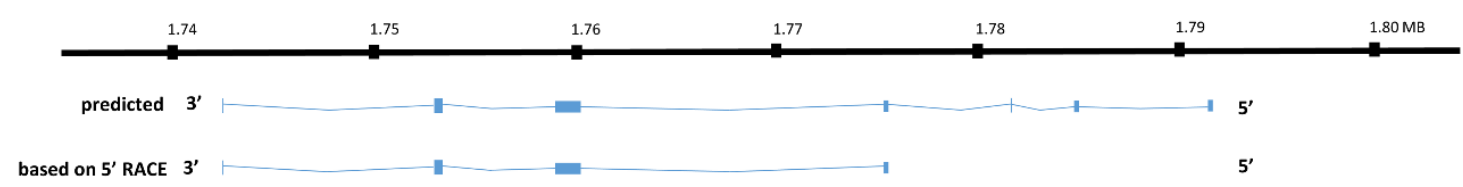

MPKGKELDETERRLILELKDVGQSNRAIAKRMGRGETVVRNELKKGENYGIRKKNKGNSKITNQFSIPFSGYGTSVREMC VQTRQLPITTSKGTTELNEKTNTTYFRNIEFNSVSPHGRLMIVGVLVNQSMTDLDAGQVRRVLHEALDLWAQNANLTFRE VYSSEADIQVLFARQFHGDGYKFDGPGKI LAHAFYPGTGIGGDAHEDEEETWLLNEPLGTEGTRLFDVAVHEFGHSLGLG HSSVKEAIMF PWHH I SYRGKDT I PEDDRLGIQG IYGPKKKTYGRNPERHTPTTTTTTTTTTLAPPRRPSTRTYYPRKDHN GHPWEGLDPNYPNYPRRTTTTSTTTEIPRRRVYFADKPRGVGTTYPTRRTYQEQPYPREPDTERPTHIPTPTKVHHHHHH HHHHRTORPVTRPPLPRI PNPCDTNYDAITI IRNELF I KKDRYLWRLFEGDSONRPPVE IDRMFFGLPKDFERIDTVYEN KHQKIVFFIGKQYYVENSQHLEPGYPKPLTHLGLPES IERVDAALVWSYNNRTYLYSGRLYWRFDEDANHVELDYPRDMS MWSGIGYNIDSAFQNRDGRTYFFKGKGYWRFNDLRMSVDHRDPHPSAERWMKCQRLPMVDITTEDQEEVVVTSAGPGSRR TEEIGG

Figure 5. Determination of the translational start site of the AeMMP2 gene by 5'RACE.

Based on 5' RACE, the ATG start site of Aemmp2 (AAEL000788) coding sequence is located in the $4^{\text {th }}$ exon (5' to3') of the currently annotated gene in VectorBase. In red: original amino acid sequence based on the current annotation of AeMMP2; in black: amino acid sequence of AeMMP2 based on 5' RACE results. 
MMP1 being most similar to HuMMPs. We performed a second maximum likelihood phylogenetic study comparing the MMP nucleotide sequences between Ae. aegypti and Ae. albopictus (Fig. 6B). It became apparent that all AeMMPs are homologues to those of Ae. albopictus (AaMMPs). AeMMP9 is an exception as it is a duplication of AeMMP1 but lacking the hemopexin domain. In Ae. albopictus, MMP genes underwent further expansion as there are two AaMMP genes homologous to Aemmp4 and two homologous to Aemmp3. The Ae. albopictus genome contains another nucleotide sequence, which is homologous to that of Aemmp1; however, the sequence only encodes the hemopexin domain (lacking catalytic and propeptide domains) and cannot be considered a functional MMP. Those MMPs of $C x$. quinquefasciatus and Ae. albopictus that are homologous to AeMMP2, AeMMP5 and AeMMP8 also lack a propeptide domain.

\section{Expression profiles of AeMMP genes during different life stages of Ae. aegypti and in females that received a CHIKV-containing bloodmeal}

We analyzed AeMMP mRNA expression levels during different mosquito life stages via qRT-PCR and compared transcript abundances to those of sugarfed female mosquitoes. We only detected expression of four of the nine AeMMP genes: Aemmp1, Aemmp2, Aemmp4 and Aemmp6 (Fig. 7 and data not shown). Generally, Aemmp1 and Aemmp2 exhibited low expression levels in the mosquito, not changing more than 10 - fold irrespective of the developmental stage of the mosquito. Aemmp1 was highly expressed in males and upregulated in females at $72 \mathrm{~h}$ post blood meal (hpbm). Aemmp1 expression also responded 


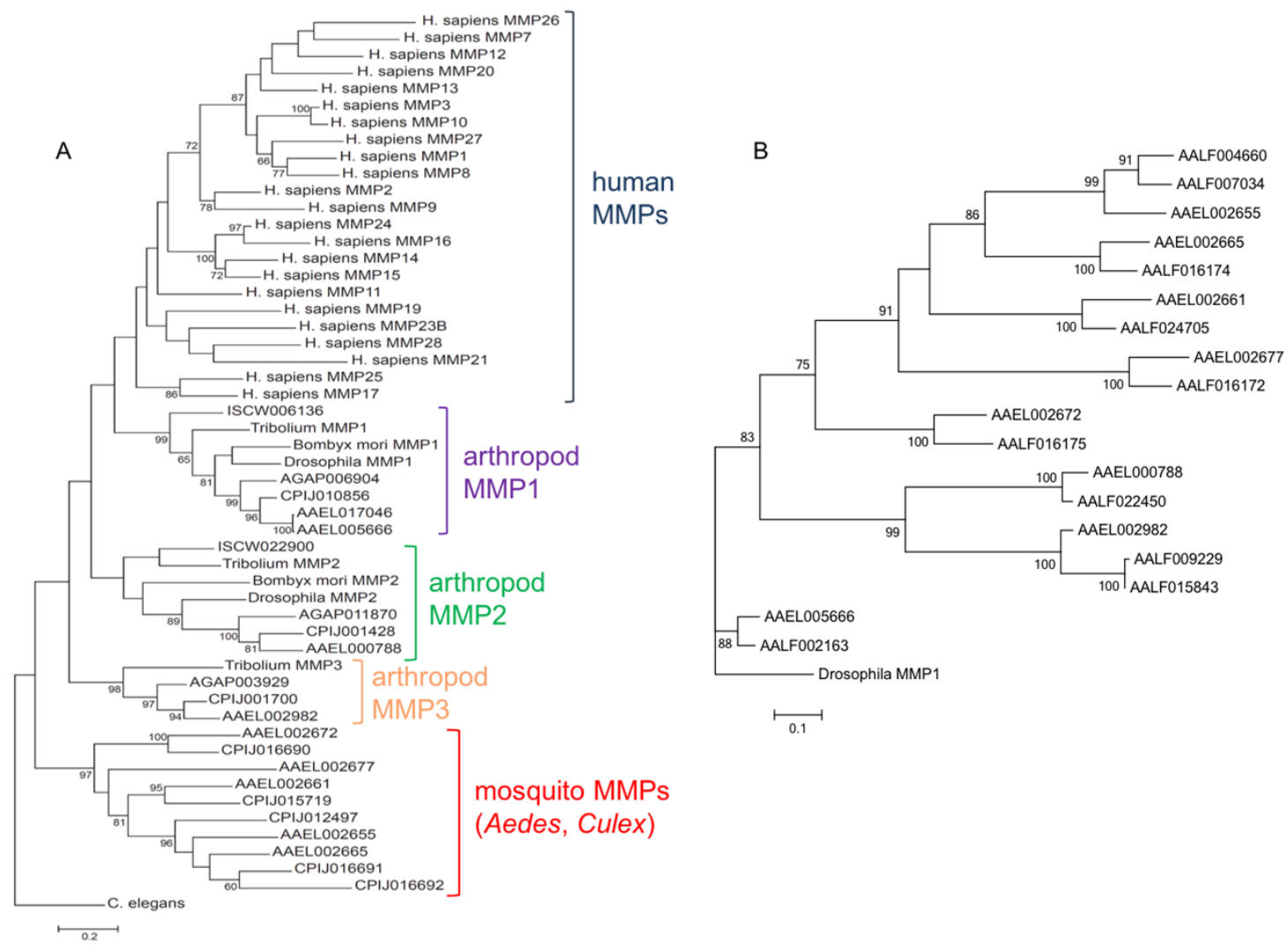

Figure 6. Phylogenetic relationships of Aedes aegypti matrix metalloproteinases (AeMMPs) with human and other arthropod MMPs.

Maximum likelihood phylogeny for (A) arthropod and human MMPs and (B) for AeMMPs and Aedes albopictus ( $\mathrm{Aa}$ )MMPs based on nucleotide sequences from which the linker encoding sequences (between catalytic and hemopexin motifs) have been removed. Bootstrap values greater than 59 are shown and indicate support as a percentage of 1000 replicates. Scale bars represent 0.2 (A) and 0.1 (B) substitutions per site. Species used for analysis: Ixodes scapularis (ISCW), Anopheles gambiae (AGAP), Culex quinquefasciatus (CPIJ), Ae. aegypti (AAEL), Aedes albopictus (AALF). 
Aemmp1
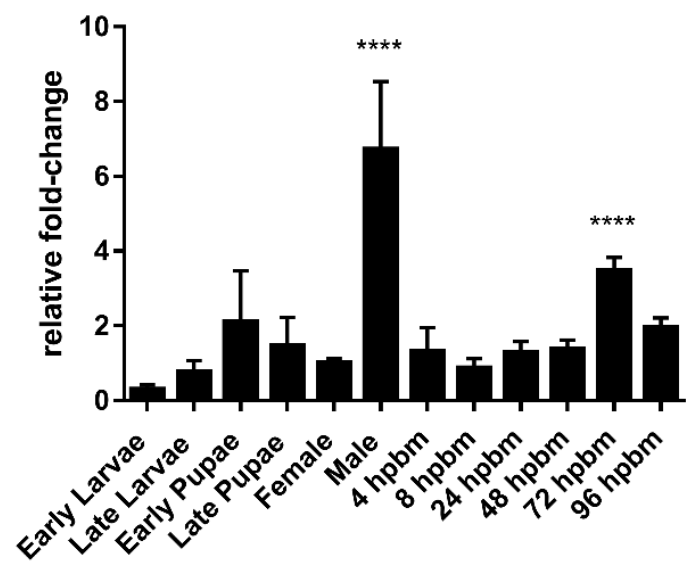

Aemmp4
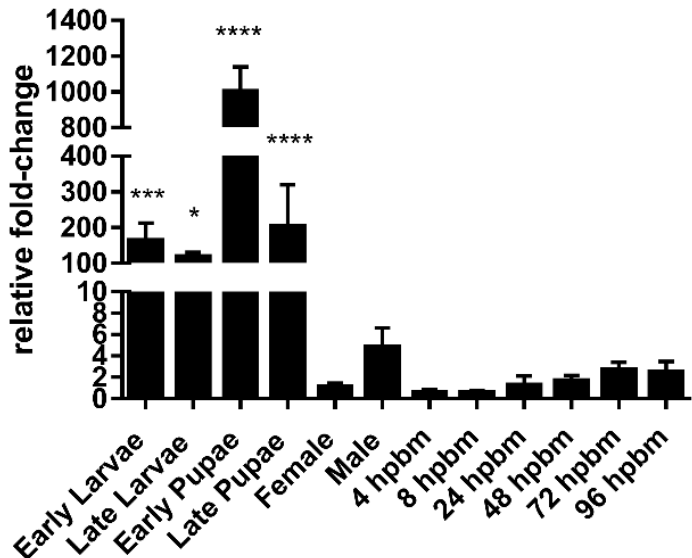

Aemmp2
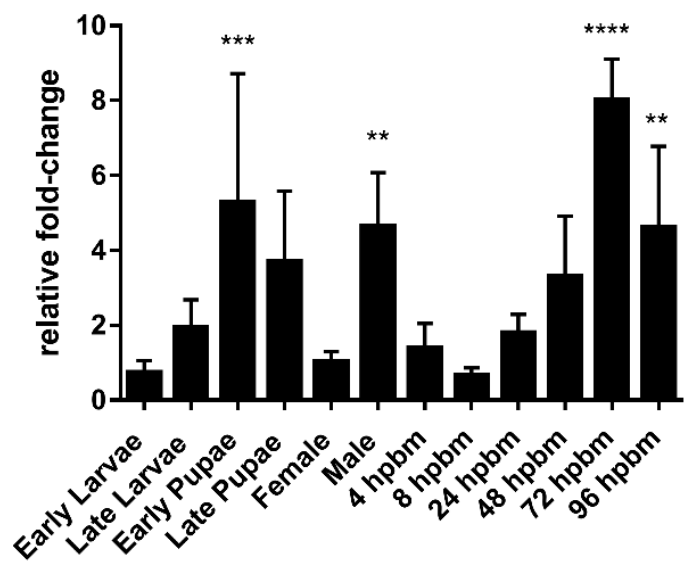

Aemmp6

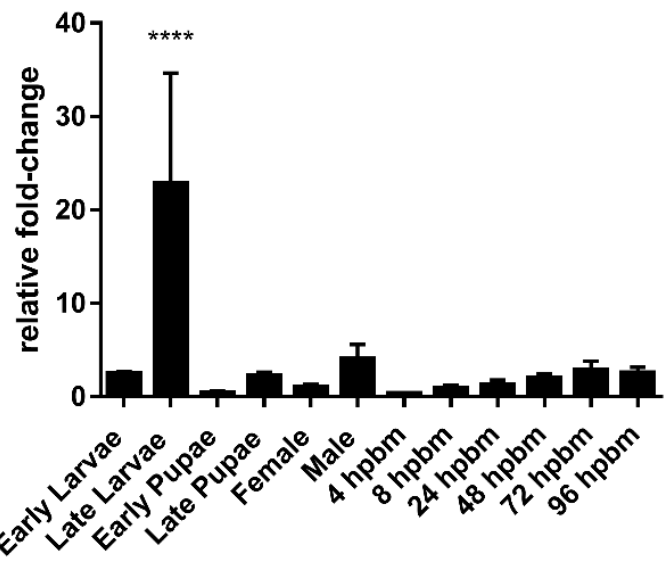

Figure 7. Expression profiles Aemmp1, Aemmp2, Aemmp4, and Aemmp6 during different life stages of Ae. aegypti.

Total RNA was extracted from HWE mosquitoes at different life-cycle stages and from wholebody females, which had received a sugarmeal or a bloodmeal (4-96 hpbm). Relative foldchange transcript abundance was analyzed by quantitative reverse transcription PCR for each AeMMP gene by normalizing reads against the abundance of ribosomal protein $\mathrm{S} 7$ transcripts. Values were then set in relation to the gene expression level in sugar-fed females (baseline value $=1$ relative fold-change). Mean values with SD from three independent experiments are shown. Statistical analysis was performed using a one-way analysis of variance followed by Tukey's multiple comparisons test ( $\left.{ }^{*}, P \leq 0.05 ;{ }^{* *}, P \leq 0.005 ;{ }^{* * *}, P \leq 0.0005 ;{ }^{* * * *}, P \leq 0.0001\right)$. 
to the presence of $\mathrm{CHIKV}$ (titer in the bloodmeal: $10^{7}$ plaque-forming units $(\mathrm{pfu}) / \mathrm{ml})$ with significantly increased gene expression in midguts at $48 \mathrm{~h}$ post infection and in carcasses at $96 \mathrm{~h}$ post infection (Fig. 8). Aemmp2 was most highly expressed in early pupae and males, and was upregulated in females at 72 and 96 hpbm (Fig. 7). However, Aemmp2 expression in either mosquito midguts or carcasses did not significantly respond to the presence of CHIKV (Fig. 8).

Aemmp4 and Aemmp6 were significantly upregulated at earlier stages of mosquito development. Aemmp4 was 100- to 1000-fold upregulated between early larva and late pupa stages, whereas Aemmp6 was upregulated 22-fold during the late larval stage of development. Expression levels of Aemmp4 and Aemmp6 were not significantly increased in the adult stage irrespectively of whether mosquitoes had received a sugarmeal, a virus-free bloodmeal or a bloodmeal containing CHIKV (Figs 5 and 6). The only exception was the significant (albeit only $\sim 2$-fold) upregulation of Aemmp6 in carcasses of CHIKVinfected mosquitoes at $96 \mathrm{~h}$ post infection. Our observations support the hypothesis that Aemmp4 and Aemmp6 may be predominantly involved in tissue remodeling during metamorphosis, whereas Aemmp1 and Aemmp2 were responsive to bloodmeal digestion in the female. Aemmp1 expression was also significantly increased in midguts and carcasses when CHIKV was present in the bloodmeal. 

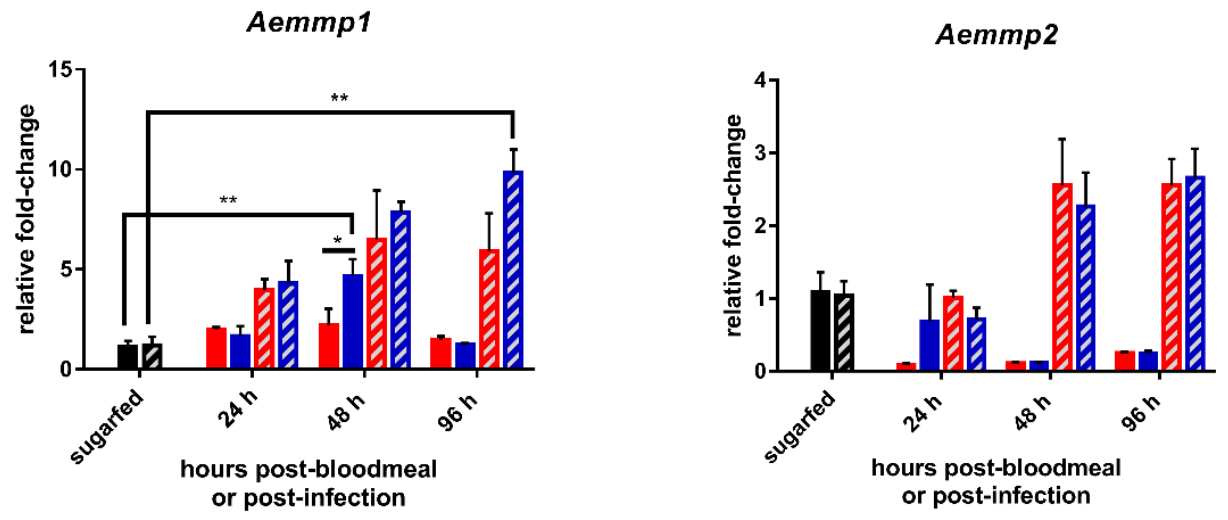

Sugar MG

DU Sugar Carcass

Blood MG

- CHIKV MG

2. Blood Carcass

a. CHIKV Carcass
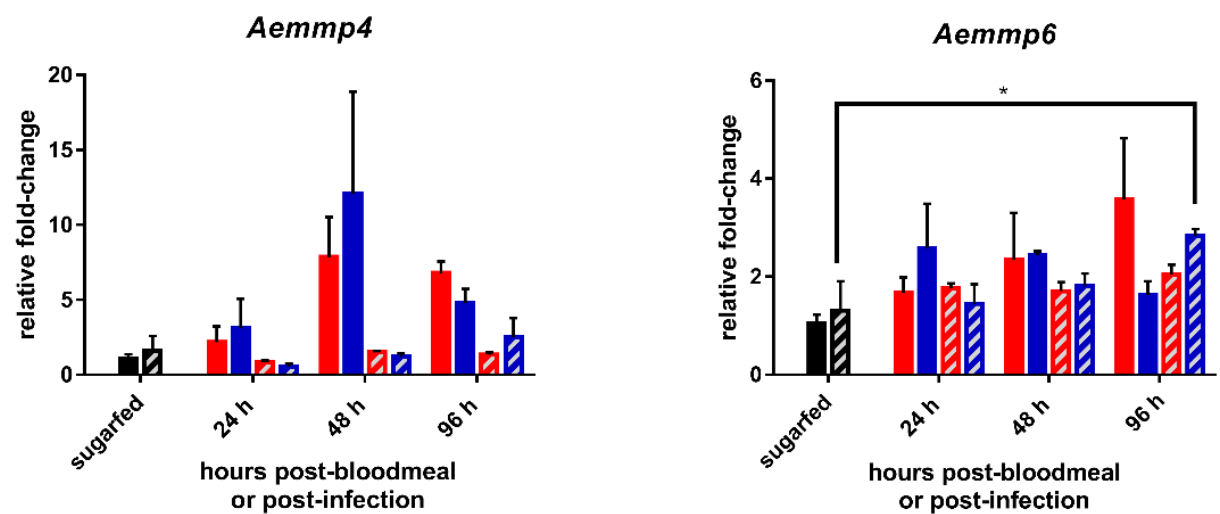

Figure 8. Expression profiles for Aemmp1, Aemmp2, Aemmp4, and Aemmp6 in midguts and carcasses of $A e$. aegypti females after a non-infectious or CHIKV containing bloodmeal.

Total RNA was extracted at 24, 48 and $96 \mathrm{~h}$ post-feeding from midguts and carcasses of HWE mosquitoes, which had received a sugarmeal, a bloodmeal diluted $(1: 1)$ with non-infected cell culture medium or a bloodmeal diluted (1:1) with culture medium from CHIKV-infected Vero cells (titer in the bloodmeal: $10^{7}$ plaque-forming units $\mathrm{pfu} / \mathrm{ml}$ ). Relative fold-change transcript abundance was analysed by quantitative reverse transcription PCR for each AeMMP gene by normalizing reads against the abundance of ribosomal protein S7 transcripts. Values were then set in relation to the gene expression level in sugar-fed midguts (baseline value $=1$ relative fold-change). Mean values with SD from three independent experiments are shown. Statistical analysis was performed using a one-way analysis of variance followed by Tukey's multiple comparisons test $\left({ }^{*}, P \leq 0.05 ;{ }^{* *}, P \leq 0.005\right)$. 


\section{Detection of AeMMP1 and AeMMP2 proteins in midguts of Ae. aegypti}

Polyclonal antibodies, pAb-mmp1 and pAb-mmp2, were produced against short peptides derived from the hemopexin domains of AeMMP1 and AeMMP2, respectively. In Western blots, pAb-mmp1 and pAb-mmp2 detected their corresponding antigens when expressed as recombinant proteins in Escherichia coli (Fig. 9). Two band signals detected by pAb-mmp1 corresponded to molecular masses of $\sim 75$ and $\sim 60 \mathrm{kDa}$ and two band signals detected by pAbmmp2 were around 75 and $65 \mathrm{kDa}$. Taking the $\mathrm{N}$-terminal protein tag sequences (Trx-tag, S-tag and His-tag) originating from the expression vector [pET-32a(+)] into account, the $75 \mathrm{kDa}$ band signals of AeMMP1 and AeMMP2 corresponded to the molecular masses of the complete AeMMP proteins; the predicted molecular masses of the three plasmid-derived tags, AeMMP1 and AeMMP2 were 15, 63 and $60 \mathrm{kDa}$, respectively. Detection of AeMMP2 with an anti-His-tag $\mathrm{Ab}$ (pAb-HisTag) resulted in a signal pattern similar to that detected with pAbmmp2, whereas pAb-HisTag failed to detect AeMMP1 recombinant protein. pAbmmp1 and pAb-mmp2 did not detect heterologous antigen or antigen in nonIsopropyl $\beta$-D-1- thiogalactopyranoside (IPTG) induced bacterial cultures. Furthermore, antigen detected by the preimmune serum (with similar intensities in IPTG- or non-induced cultures) was distinct from that detected by pAb-mmp1 and pAb-mmp2. These observations suggest that both antibodies specifically detected their homologous AeMMP antigens. 

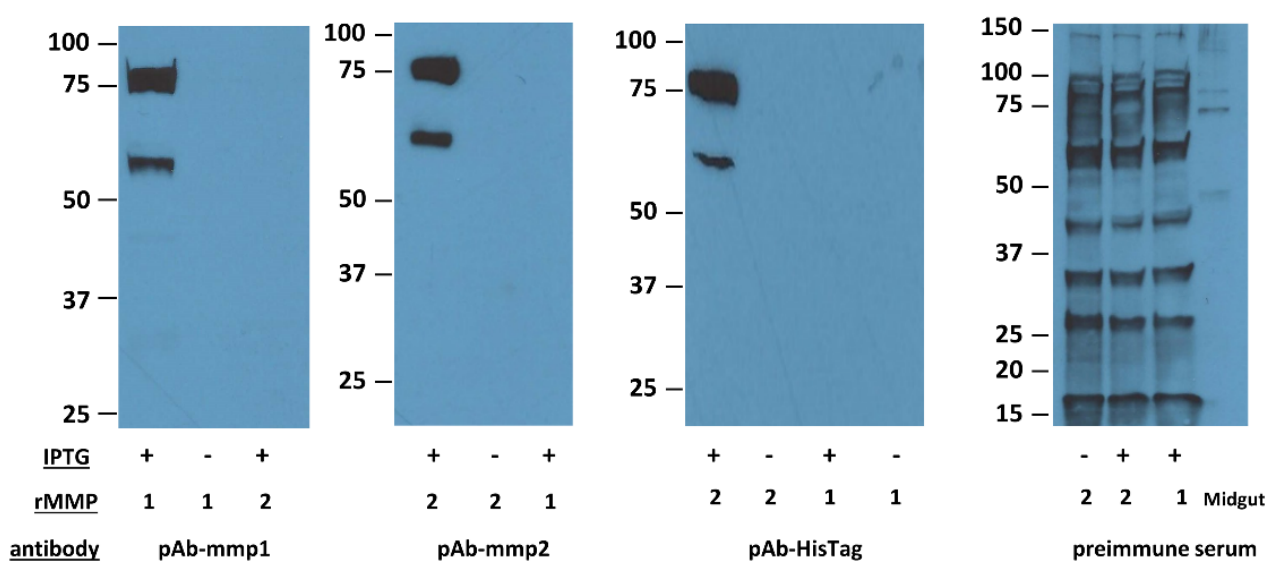

Figure 9. Validation of polyclonal antibodies pAb-mmp1 and pAb-mmp2.

Polyclonal antibodies pAb-mmp1 and pAb-mmp2 (in comparison to pAb-HisTag or preimmune serum) used to detect recombinant AeMMP1-His and AeMMP2-His following IPTG-mediated induction/no induction of recombinant protein production. Data in the figure are representative of experiments that have been repeated three times. 
In midguts of Ae. aegypti, AeMMP1 was detected with similar intensities at 24 and $72 \mathrm{hpbm}$ or CHIKV infection (virus titer in the bloodmeal: $10^{7} \mathrm{pfu} / \mathrm{ml}$ ) (Fig. 10A). At $48 \mathrm{hpbm} / \mathrm{CHIKV}$ infection, AeMMP1 antigen seemed to be less abundant, although the $\beta$-actin loading control was also slightly reduced. Two band signals with molecular masses of 50 and $37 \mathrm{kDa}$ were detected in mosquito midgut extracts by pAb-mmp1. According to molecular mass predications, the 50 $\mathrm{kDa}$ band possibly corresponded to the zymogen form of AeMMP1, whereas the $37 \mathrm{kDa}$ band could represent its active form after cleavage of its $\sim 15 \mathrm{kDa}$ propeptide domain. This indicates that upon its expression in midguts, AeMMP1 may be rapidly proteolytically processed, for example via furin cleavage (see also Fig. 4A) as has been observed for AgMT-MMP1 in mosquito cells (140). pAbmmp2 detected in midgut tissue two closely migrating band signals of around 75 and $70 \mathrm{kDa}$, which were larger than the predicted molecular mass of AeMMP2 protein (Fig. 10B). We speculate that post-translational modifications such as protein glycosylation could account for this molecular mass discrepancy. AeMMP2 antigen was less detectable at 24 and $48 \mathrm{hpbm}$ compared to $72 \mathrm{hpbm}$ or in midguts of sugar-fed mosquitoes. Similar to AeMMP1, the presence of CHIKV did not obviously affect the abundance of AeMMP2 antigen. 
A

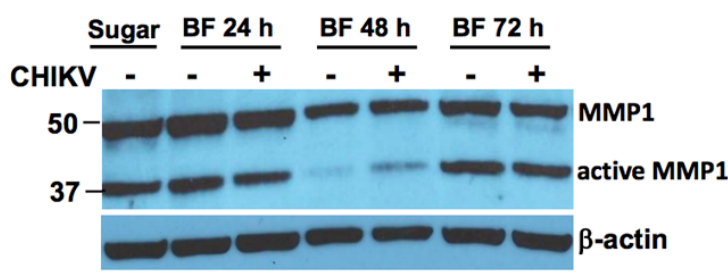

B

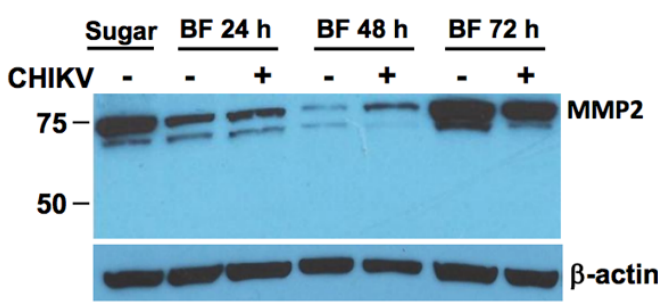

Figure 10. Detection of AeMMP1 and AeMMP2 antigens by Western blot analysis.

(A) Western blot detection of AeMMP1 antigen by pAb-mmp1 and (B) AeMMP2 antigen by pAb-mmp2 in midguts of mosquitoes that had received a sugarmeal, a bloodmeal diluted 1:1 with non-infected cell culture medium or a bloodmeal diluted 1:1 with chikungunya virus (CHIKV)-infected cell culture medium (titer in the bloodmeal: $10^{7}$ plaque-forming units $\mathrm{pfu} / \mathrm{ml}$ ) at 24,48 and $72 \mathrm{~h}$ postfeeding (BF). $\beta$-actin was used as a loading control. Molecular masses in $\mathrm{kDa}$ are indicated. Data in the figure are representative of experiments that were repeated twice. 


\section{AeMMP1 is enzymatically active in vitro}

We established the catalytic activity of recombinant AeMMP1 in vitro. After treatment with proMMP activator 4-aminophenylmercuic acetate (AMPA) recombinant AeMMP1 was incubated with a generic MMP substrate, FS-6. The peptide MCA-Lys-Pro-Leu-Gly-Leu-DNP-Dpa-Ala-Arg-NH2, or FS-6, is a fluorogenic substrate with improved kinetic properties for several MMPs including HuMMP1(201). Recombinant AeMMP1 was able to cleave this substrate in vivo and the catalytic activity was able to be diminished when the broad spectrum MMP inhibitor GM6001 was introduced (Fig. 11 A,B). Recombinant AeMMP1 activity was also inhibited by EDTA and by human Tissue Inhibitor of MMP (TIMP)3, which is the closest homolog of the single TIMP encoded in the Ae. aegypti genome.

\section{Localization of AeMMP1 and AeMMP2 in midguts via IFA}

In immunofluorescence assays, AeMMP1 was detected in epithelial tissue of the midgut and in midgut-associated muscle fibers but not in tracheal cells, whereas AeMMP2 was only detectable in midgut- associated tracheal cells (Fig. 12). Acquisition of a bloodmeal or the presence of CHIKV in the bloodmeal did not obviously affect the abundance of either AeMMP antigen in midgutassociated tissues or the tissue specificity of either AeMMP (Fig. 13). AeMMP1 antigen, like CHIKV antigen, was associated with epithelial tissue and both antigens co-localized in the tissue at $96 \mathrm{~h}$ post infection. AeMMP2 antigen was not detected in epithelial cells where CHIKV infection occurred. 
A

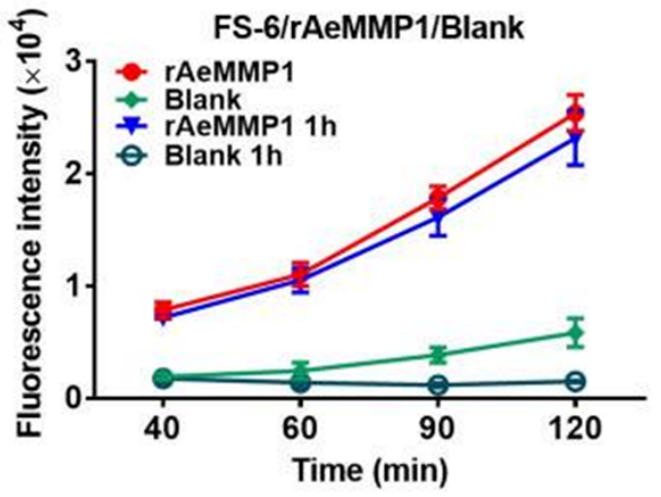

B

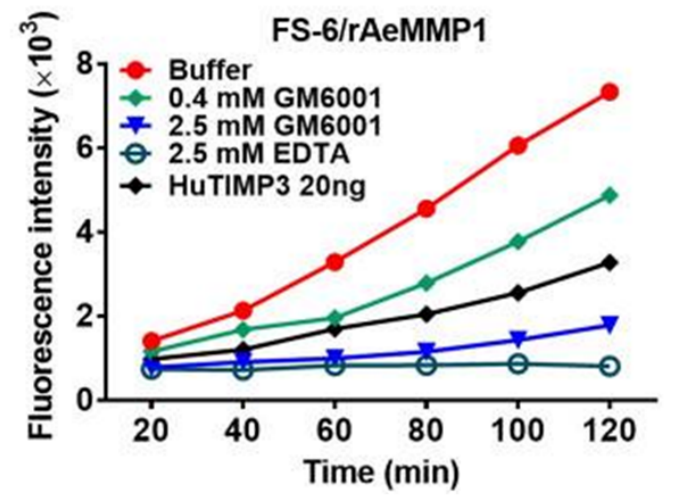

Figure 11. Kinetics of recombinant AeMMP1 activity in an in vitro MMP activity assay using FS-6 as substrate.

(A) $20 \mathrm{ng}$ of rAeMMP1 were preincubated with $1 \mathrm{mM}$ proMMP activator 4-aminophenylmercuric acetate (AMPA) or reaction buffer for $1 \mathrm{~h}$ before start of the activity assay. Blank, rAeMMP1: cell culture medium transfected with "empty" plasmid vector and rAeMMP1 expression plasmid, respectively; blank $1 \mathrm{~h}$, rAeMMP1 $1 \mathrm{~h}$ : medium was preincubated for $1 \mathrm{~h}$ with $1 \mathrm{mM}$ AMPA before FS-6 substrate incubation. (B) Kinetics of GM6001, EDTA or HuTIMP3-mediated inhibition of rAeMMP1. $20 \mathrm{ng}$ of HuTIMP3, 0.4 mM GM6001, 2.5 mM GM6001, or 2.5 mM EDTA were preincubated with $10 \mathrm{ng}$ of rAeMMP1 at RT for $2 \mathrm{~h}$ followed by addition of FS- 6 substrate. The fluorescence intensity was measured every $20 \mathrm{~min}$. 

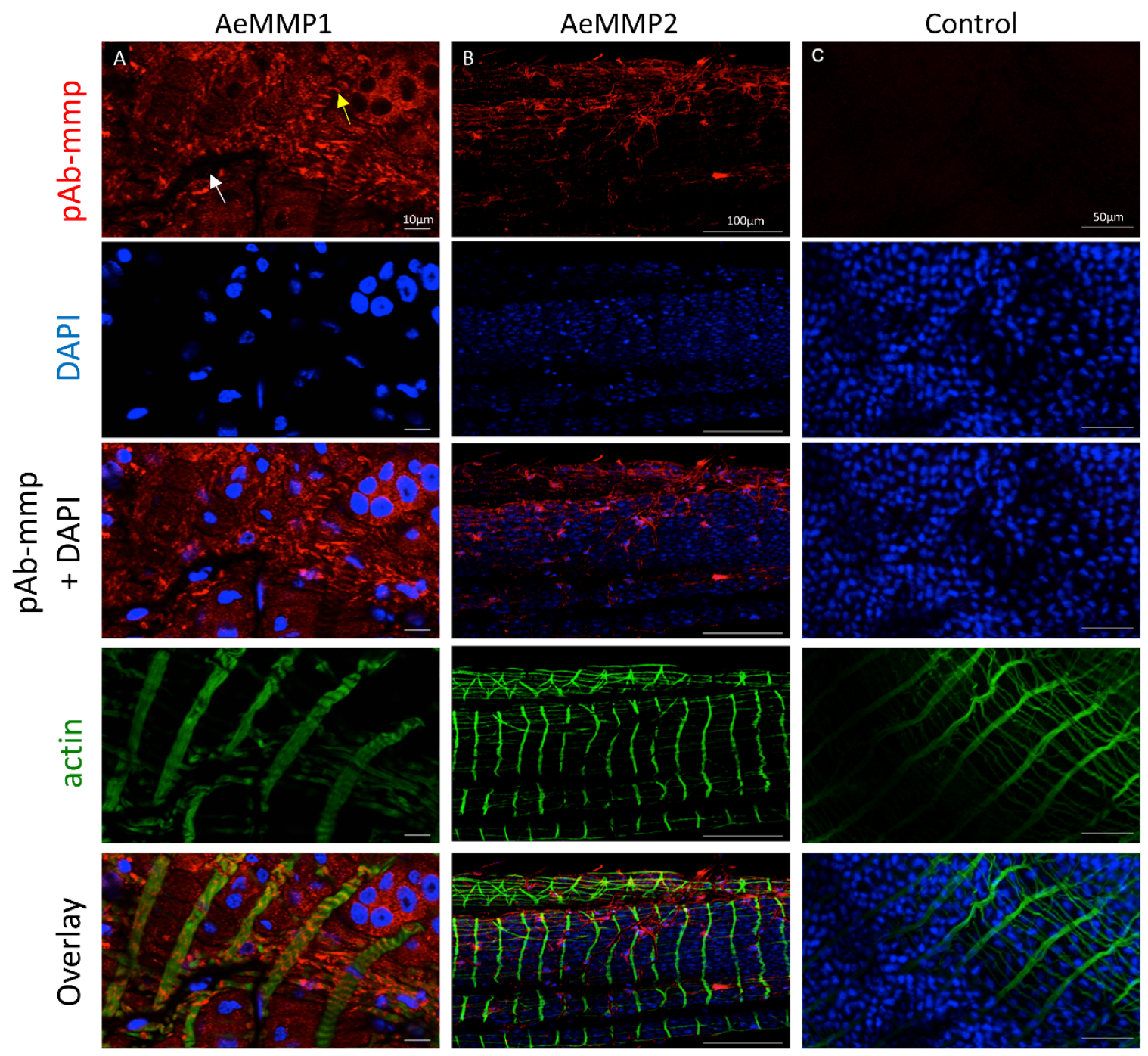

Figure 12. Detection of AeMMP1 and AeMMP2 antigens in midguts of sugar-fed Ae. aegypti by IFA.

Midguts were incubated with either (A) Polyclonal antibody (pAb)-mmp1, (B) pAb-mmp2 or (C) control with no pAb-mmp in an immunofluorescence assays using Alexa Fluor 594 labelled (red) antirabbit IgG as secondary antibody. Cell nuclei were stained with DAPI (blue) and actin was stained with Alexa Fluor Phalloidin 488 (green). White and yellow arrows indicate midgutassociated tracheal cells and muscle fibres, respectively. Scale bars: $(\mathbf{A}, \mathbf{B})=10 \mu \mathrm{m},(\mathbf{C})=50 \mu \mathrm{m}$. Confocal microscopy was performed using an inverted spectral confocal microscope (TCP SP8 MP, Leica Microsystems). Selected images are representative of six replicates. 


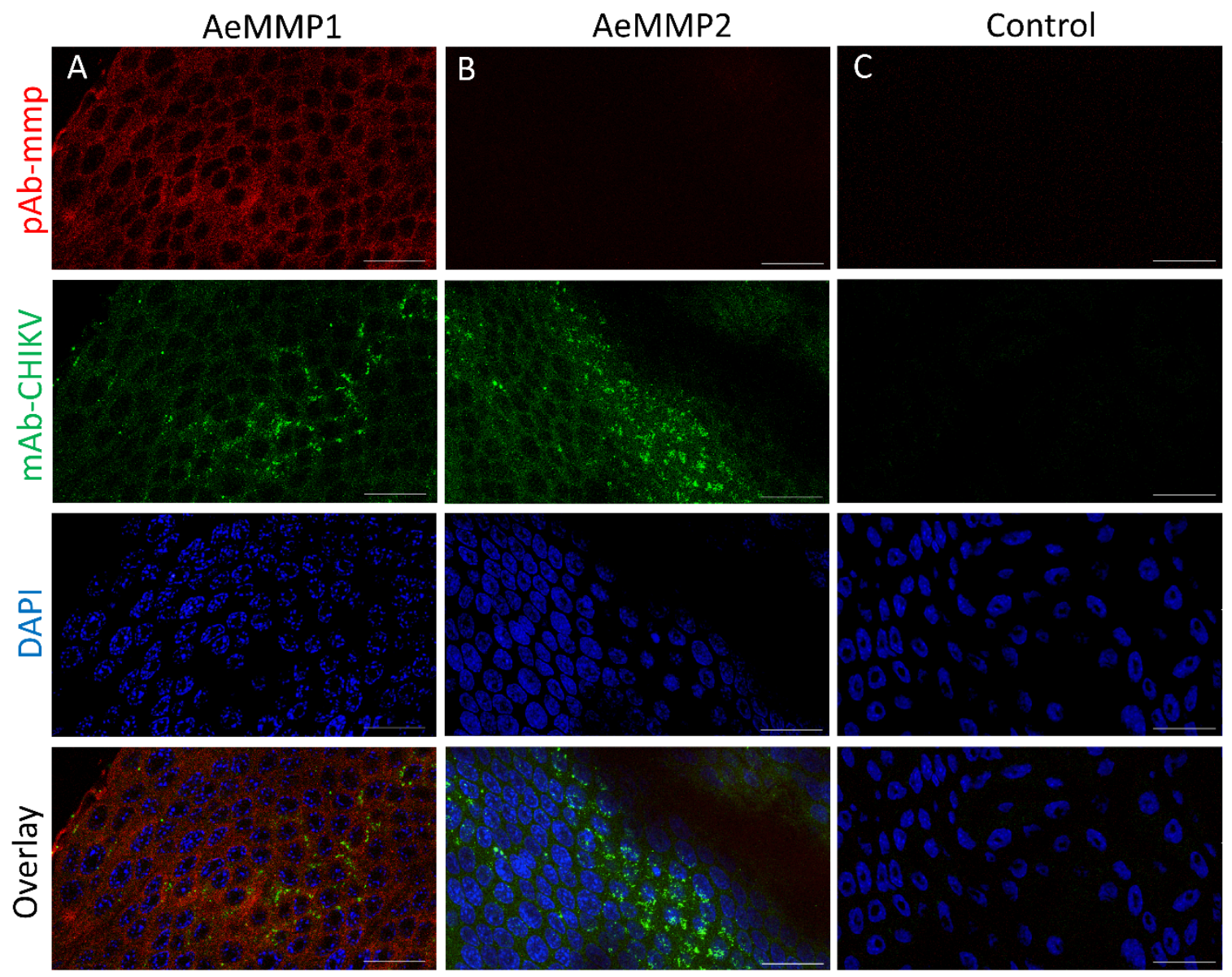

Figure 13. Detection of AeMMP1 and AeMMP2 antigens in midguts of chikungunya virus (CHIKV)-infected Ae. aegypti by IFA.

Midguts were incubated with (A, B) anti-CHIKV monoclonal antibody (mAb-CHIKV) at $96 \mathrm{~h}$ postinfection and either (A) polyclonal antibody (pAb)-mmp1, (B) pAb-mmp2 or (C) control with no $\mathrm{mAb}-\mathrm{CHIKV}$ or pAb-mmp in an immunofluorescence assay using Alexa Fluor 488 labelled (green) antimouse $\operatorname{lgG}$ and Alexa Fluor 594 labelled (red) antirabbit IgG as secondary antibodies. Cell nuclei were stained with DAPI (blue). Scale bars $=25 \mu \mathrm{m}$. Confocal microscopy was performed using an inverted spectral confocal microscope (TCP SP8 MP, Leica Microsystems). Selected images are representative of three replicates. 


\section{Discussion}

In this study, we initially characterized the MMPs of Ae. aegypti to identify those that may play a role in midgut $\mathrm{BL}$ remodeling during bloodmeal digestion and arbovirus dissemination. Arthropods have two to three MMPs in common, whereas mosquitoes of the subfamily Culicinae that have been examined so far possess an additional five (Cx. quinquefasciatus) to seven (Ae. albopictus) MMPs, which all are evolutionally distinct from HuMMPs. In Ae. aegypti, the majority of the nine MMPs contained the canonical propeptide, catalytic and hemopexin domains. Generally, propeptide and hemopexin domains are highly variable amongst MMPs and those of Ae. aegypti are no exception $(192,197)$. In contrast, the catalytic domains were highly similar amongst AeMMPs. This may be an indication that according to hemopexin and propeptide domain diversities, each AeMMP is targeting a specific type of extracellular substrate and is activated under a specific circumstance $(137,202)$.

Overall, MMP activity is tightly regulated at several levels; at the transcriptional level (MMP expression), via propeptide cleavage (MMP activation), via interaction with a tissue inhibitor of MMP protein (TIMP; MMP inhibition) and eventually through protease degradation (124). The Ae. aegypti genome, like that of other arthropods, encodes a single TIMP gene (AAEL013525), which is most similar to human TIMP3 $(137,196,203,204)$. In qRT-PCR experiments, we obtained expression data based on fold-change in transcript abundance for four of the nine AeMMP genes: Aemmp1, Aemmp2, Aemmp4 and Aemmp6. For the remaining five AeMMP genes, transcripts were 
not detected by reverse transcription PCR (RT-PCR) in whole-body mosquitoes after testing several gene-specific primer combinations (data not shown). Specific detection of Aemmp9 was not possible as its nucleotide sequence, although being truncated, is nearly identical to that of Aemmp1. A previous wholetranscriptome analysis (RNA-seq) showed that Aemmp3, Aemmp5, Aemmp7 and Aemmp8 were expressed in whole-body females of the Liverpool strain of Ae. aegypti; however, Aemmp3 and Aemmp8 exhibited only very low expression levels, hardly exceeding 10 fragments per kilobase of transcript per million mapped reads (205). Our qRT-PCR analysis supports the conclusion that Aemmp4 and Aemmp6 expression was strongly induced during metamorphosis, whereas Aemmp1 and Aemmp2 expression was induced in the female during bloodmeal digestion. Thus, AeMMP1 and AeMMP2 could potentially be involved in the dissemination process of CHIKV from the midgut. This prompted us to produce antibodies specific to each of the two AeMMPs, allowing us to further analyze their presence and activity in specific tissues at the protein level.

The Drosophila orthologue of AeMMP1 is secreted whereas the An. gambiae orthologue of AeMMP1 is alternatively spliced and either transmembrane-linked (MT-MMP) to be expressed in epithelial tissues when possessing a C-terminal GPI anchor or secreted from hemocytes of the mosquito when lacking the GPI anchor $(133,140)$. Our study did not reveal that $A e$. aegypti, like An. gambiae, expresses two isoforms of MMP1; however, according to in silico prediction tools, AeMMP1 possesses a GPI anchor. Functional analysis of $D$. melanogaster MMP1 (DmMMP1) and AgMT-MMP1 revealed that 
both are involved in basement membrane remodeling, including fibronection/collagen IV degradation, tissue repair and wound healing $(133,135,206-208)$. Upregulation of DmMMP1 during wound healing occurs via the extracellularly regulated jun $\mathrm{N}$-terminal kinase signaling pathway.

In An. gambiae, AgMT-MMP1 responded strongly to Plasmodium ookinete invasion of midgut epithelial cells and also to nonparasite-containing bloodmeal acquisition (140). Changes in AgMT-MMP1 activity were manifested not only at the transcriptional level but also at the level of zymogen cleavage and subcellular activation. As ookinete invasion leads to tissue damage, AgMT-MMP1 may be strongly activated to facilitate wound healing. Furthermore, AgMT-MMP1 activity may be required to enable midgut tissue overstretching during bloodmeal acquisition and/or tissue regeneration following bloodmeal digestion (140). Arboviruses such as CHIKV have not yet been observed to actively penetrate and damage the ECM during their infection cycle in the mosquito vector. However, midgut infection and dissemination of arboviruses are associated with the ingestion of a bloodmeal. CHIKV dissemination from the midgut has been observed as early as $24 \mathrm{~h}$ postoral acquisition of the virus, a time point at which the epithelial tissue of the blood-filled midgut was still over- stretched (14). Thus, it appears that the virus was already disseminating from the midgut before transcriptional upregulation of Aemmp1 had reached its peak, which occurred at around $72 \mathrm{hpbm}$, a time point at which the bloodmeal was already digested. Similarly, Aemmp2 expression levels were significantly increased in blood-fed mosquitoes towards the end of bloodmeal digestion with increased gene 
expression occurring outside the midgut. In situ studies showed that AeMMP1 was abundant in midgut epithelial cells and muscle fibers whereas AeMMP2 was associated with tracheal cells. Both AeMMP antigens were readily detected in midgut tissues of sugar-fed mosquitoes and antigen abundance did not seemingly increase over a 96-h period following ingestion of a bloodmeal containing (or not containing) CHIKV with the exception of AeMMP2 at 72 hpbm. Based on these observations, we speculate that at the end of bloodmeal digestion, increased AeMMP1 and AeMMP2 production may have occurred in tissues outside the midgut epithelium for potential ECM remodeling required after the bloodmeal digestion process to restore overexpanded tissues to their original size. Although we do not see evidence of AeMMP upregulation until late during bloodmeal digestion, we cannot rule out the possibility that AeMMP activity may be essential for CHIKV dissemination from the midgut. Additional, functional studies will be needed to test these hypotheses. 


\section{CHAPTER IV}

\section{Ultrastructural Analysis of Chikungunya Virus Dissemination from the Midgut of the Yellow Fever Mosquito, Aedes aegypti}

This chapter has been published in the journal, Viruses

Asher M. Kantor, ${ }^{1}$ DeAna G. Grant, ${ }^{2}$ Velmurugan Balaraman, ${ }^{1}$ Tommi A. White, ${ }^{2,3}$ and Alexander W. E. Franz ${ }^{1}$. "Ultrastructural Analysis of Chikungunya Virus Dissemination from the Midgut of the Yellow Fever Mosquito, Aedes Aegypti." Viruses 10.10 (2018): n. pag. Web.

AMK, TAW, and AWEF conceived and designed the experiments; AMK, DGG, and VB performed the experiments; AMK, DGG, VB, TAW, and AWEF analyzed the data; DGG and TAW contributed reagents/materials/analysis tools; AMK and AWEF wrote the paper.

${ }^{1}$ Department of Veterinary Pathobiology, University of Missouri, Columbia, MO 65211, USA

${ }^{2}$ Electron Microscopy Core Facility, University of Missouri, Columbia, MO 65211, USA

${ }^{3}$ Department of Biochemistry, University of Missouri, Columbia, MO 65211, USA 


\section{Abstract}

The transmission cycle of chikungunya virus (CHIKV) requires that mosquito vectors get persistently infected with the virus, following its oral acquisition from a vertebrate host. The mosquito midgut is the initial organ that gets infected with orally acquired CHIKV. Following its replication in the midgut epithelium, the virus exits the midgut and infects secondary tissues including the salivary glands before being transmitted to another host. Here, we investigate the pattern of CHIKV dissemination from the midgut of Aedes aegypti at the ultrastructural level. Bloodmeal ingestion caused overstretching of the midgut basal lamina $(\mathrm{BL})$, which was disrupted in areas adjacent to muscles surrounding the midgut as shown by scanning electron microscopy (SEM). Using both transmission electron microscopy (TEM) and focused ion beam scanning electron microscopy (FIB-SEM) to analyze midgut preparations, mature chikungunya $(\mathrm{CHIK})$ virions were found accumulating at the $\mathrm{BL}$ and within strands of the $B L$ at $24-32 \mathrm{~h}$ post-infectious bloodmeal (pibm). From $48 \mathrm{~h}$ pibm onwards, virions no longer congregated at the $\mathrm{BL}$ and became dispersed throughout the basal labyrinth of the epithelial cells. Ingestion of a subsequent, non-infectious bloodmeal caused mature virions to congregate again at the midgut $\mathrm{BL}$. Our study suggests that CHIKV needs a single replication cycle in the midgut epithelium before mature virions directly traverse the midgut $B L$ during a relatively narrow time window, within $48 \mathrm{~h}$ pibm. 


\section{Introduction}

Chikungunya virus (CHIKV) is an Old World alphavirus

(family: Togaviridae) belonging to the Semliki Forest virus sero-complex (22).

Originating from Africa, the virus is also highly prevalent in Southeast Asia, including the Indian subcontinent $(187,209)$. At the end of 2013, CHIKV emerged in the Western Hemisphere, initially in the Caribbean followed by outbreaks in Brazil $(210,211)$. Meanwhile, the virus has been reported in most countries of South and Central America, including sporadic incidences in the southern United States. Typical chikungunya ( $\mathrm{CHIK})$ disease symptoms in humans include febrile illness, rash, myalgia, joint swelling, and arthralgia, which can be long-lasting (187). Like all other alphaviruses, CHIKV is mosquito-borne, with Aedes aegypti and $A$ e. albopictus functioning as the principal CHIKV vectors in urban transmission cycles.

A mosquito needs to be persistently infected with an arbovirus, such as $\mathrm{CHIKV}$, in order to transmit the virus to susceptible hosts. The mosquito midgut consists of a single layer of epithelial cells surrounded at the basal side by a multi-stranded basal lamina $(\mathrm{BL})$ predominantly consisting of laminin and collagen IV $(114,115,189)$. The anterior portion of the midgut takes part in sugar absorption whereas the posterior region is responsible for bloodmeal digestion. Following the ingestion of a viremic bloodmeal from a CHIKV infected human, the virus enters the lumen of the mosquito midgut wherein the bloodmeal is digested. Before the bloodmeal is surrounded by the peritrophic matrix, virions need to 
enter the midgut epithelial cells via receptor-mediated endocytosis $(189,212)$. At the ultrastructural level, the following zones can be distinguished in a cross section through a midgut epithelial cell: prominent microvilli for nutrient uptake at the luminal side, an endoplasmic reticulum (ER) surrounding the nucleus, and a prominent basal labyrinth, which is a network of intracellular spaces located towards the cell's basal side adjacent to the BL $(106,114,115,189,213)$.

The mechanism underlying the viral exit from the midgut is not well understood $(121,150,189)$. Most of the previous ultrastructural studies in which arbovirus dissemination from the midgut was investigated were conducted with Culex spp., which had been infected with flaviviruses, alphaviruses, or bunyaviruses $(144,149,214-218)$. Flaviviruses, such as St. Louis encephalitis virus (SLEV) or West Nile virus (WNV), and bunyaviruses, such as Rift Valley fever virus (RVFV), employ different replication and virion assembly strategies in an infected cell than alphaviruses $(219,220)$. Earlier observations also suggested that alphaviruses in general disseminate more rapidly from the mosquito midgut than flaviviruses or bunyaviruses $(215,221)$. Other ultrastructural studies on alphavirus mosquito infections did not focus on the viral dissemination mechanism (222-228). Apart from exiting the posterior midgut through the $\mathrm{BL}$, several studies describe that arboviruses also infect the anterior midgut region, including the intussuscepted foregut/cardia $(144,229-234)$. However, the role of intussuscepted foregut/cardia infection in virus dissemination to secondary tissues is not clear (189) and so far, infection of these tissues with CHIKV has not been reported. 
Numerous reports describe the phenomenon of a midgut escape barrier causing virus dissemination from the mosquito midgut to be inefficient or delayed although the midgut epithelium is strongly infected $(214,235-239)$. Furthermore, the $\mathrm{BL}$ surrounding the midgut has been proposed as the principal barrier to arbovirus dissemination, including that of CHIKV $(121,150,240)$. The pore size exclusion limit for the BL of a mosquito midgut has been estimated to be just 10 nm, whereas CHIK virions have a diameter of $60-70 \mathrm{~nm}(106,121,212,241,242)$. Thus, in order for virions to pass through the BL, its pore size exclusion limit needs to be (at least temporarily) enlarged. Recently, we investigated the role of matrix-metalloproteinases (MMPs) as a group of enzymes potentially involved in $\mathrm{BL}$ remodeling during bloodmeal digestion and $\mathrm{CHIKV}$ dissemination from the mosquito midgut $(150,240,243)$. Our group has also demonstrated the principal barrier character of the BL to CHIKV. Intrathoracically injected virus was plausibly unable to infect the midgut of sugarfed mosquitoes and virions therefore accumulated at the basal (hemocoel) side of the midgut-surrounding BL (150). The female mosquito deposits an ingested sugarmeal into her crop instead of directing it immediately to the midgut. However, intrathoracically injected $\mathrm{CHIK}$ virions were readily able to infect the midgut epithelium when there was a bloodmeal present in the midgut lumen. Furthermore, substituting a bloodmeal for a protein (bovine serum albumin) or saline (phosphate buffer saline) meal did not negatively affect the ability of orally acquired CHIKV to disseminate from the midgut (240). Taken together, these observations allow the conclusion that 
stretching of the gut tissue may be the critical factor causing the BL to become permissive for the virus.

The purpose of this study was to closely observe the dissemination pattern of CHIKV from the midgut over time at the ultrastructural level. We used scanning electron microscopy (SEM) to visualize to what extent bloodmeal ingestion caused overstretching and damage to the midgut $\mathrm{BL}$. We conducted a transmission electron microscopy (TEM) based time course study to reveal whether or not $\mathrm{CHIK}$ virions continuously disseminated from the midgut and which route of dissemination the virus utilized in Ae. aegypti. Using a focused ion beam scanning electron microscope (FIB-SEM) to perform three-dimensional tomography (244-248), we visually confirmed the midgut escape route of CHIKV. We investigated the effect of a consecutive (non-infectious) bloodmeal on the dissemination of $\mathrm{CHIKV}$, which had been orally acquired along with an initial bloodmeal. The observed dissemination pattern of the virus was molecularly confirmed using a highly sensitive viral RNA strand-specific qRT-PCR assay in which we included Mayaro virus (MAYV) to compare its replication dynamics in mosquito tissues with those of CHIKV. MAYV is another alphavirus of the Semliki Forest virus sero-complex resembling CHIKV in terms of clinical disease symptoms in humans and vector interaction with Ae. aegypti under laboratory conditions (32). MAYV is prevalent in the Western Hemisphere, circulating in sylvatic cycles in rain forest areas of Central- and South America. So far, a CHIKV-like urban disease cycle involving Ae. aegypti has not been reported for MAYV (249). 


\section{Results}

\section{Bloodmeal ingestion causes temporal midgut tissue expansion and damage to the $B L$}

In laboratory maintained HWE mosquito females, a pure bloodmeal consisting of undiluted defibrinated sheep blood was digested within 3 days postoral acquisition via membrane feeding (Fig. 14). As shown in Table 4, the midgut tissue of a fully engorged female was $20 \times$ expanded based on an average increase in volume from $0.2 \mu \mathrm{l}$ (midgut of a sugarfed female) to $3.9 \mu \mathrm{l}$ (midgut of a bloodfed female). As a consequence of nutrient absorption and desiccation, the volume of the bloodmeal gradually shrank until almost completely digested at 72 h post-(non-infectious) bloodmeal (pbm) (Fig. 14). Concurrently, the overstretched midgut tissue contracted until the pre-bloodfeeding condition was reached again between 72 and $96 \mathrm{~h} \mathrm{pbm}$.

Ultrastructural imaging of the midgut surface during bloodmeal digestion via SEM revealed the overstretching of the BL surrounding the bloodfed, and/or CHIKV-infected midgut (Fig. 15). The midgut surface structure of sugarfed mosquitoes appeared relaxed and consisted of deep invaginations with clusters of epithelial cells bulging out from the surface. As indicated by the red arrows in Fig. 15, occasional slight tears in the BL were visible, which were not as prominent and regularly apparent as in the bloodfed midgut samples (exemplified by white arrows in Fig. 15). At $8 \mathrm{~h}$ post-infectious bloodmeal (pibm), the BL was 


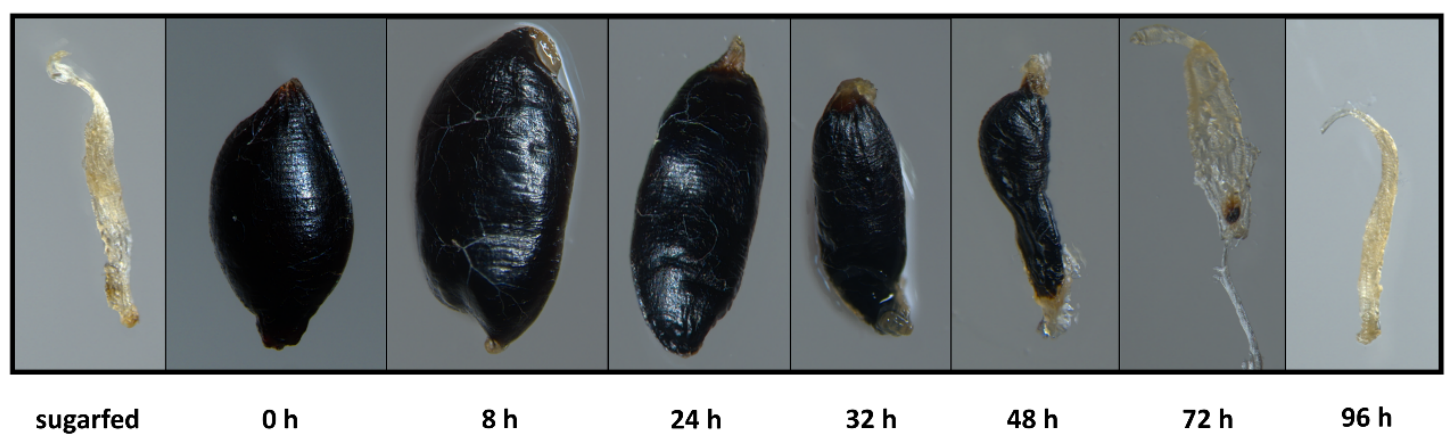

Figure 14. Visual comparison of bloodmeal digestion over time in the midgut of Ae. aegypti.

One week-old Higgs White Eye (HWE) females ingested an artificial bloodmeal consisting of undiluted defibrinated sheep blood. Light microscope magnification: 30x. 
Table 4. Sizes and volumes of midguts obtained from sugarfed and bloodfed (immediately after bloodfeeding) Ae. aegypti.

\begin{tabular}{|c|c|c|c|}
\hline Sugarfed female & $\begin{array}{c}\text { Midgut diameter } \\
(\mathrm{mm})\end{array}$ & $\begin{array}{c}\text { Migut length } \\
(\mathrm{mm})\end{array}$ & $\begin{array}{c}\text { Midgut volume } \\
(\mu \mathrm{l})\end{array}$ \\
\hline$\# 1$ & 0.4 & 2.0 & 0.25 \\
\hline$\# 2$ & 0.3 & 1.2 & 0.08 \\
\hline$\# 3$ & 0.4 & 1.6 & 0.20 \\
\hline$\# 4$ & 0.4 & 2.0 & 0.25 \\
\hline$\# 5$ & 0.3 & 1.6 & 0.11 \\
\hline$\# 6$ & 0.4 & 1.5 & 0.19 \\
\hline$\# 7$ & 0.4 & 2.0 & 0.25 \\
\hline$\# 8$ & 0.4 & 1.6 & 0.20 \\
\hline$\# 9$ & 0.4 & 2.0 & 0.25 \\
\hline$\# 10$ & 0.4 & 1.6 & 0.20 \\
\hline & & & average 0.199 \\
\hline & & & std. deviation 0.059 \\
\hline bloodfed female & Midgut diameter & Migut length & Midgut volume \\
\hline & $(\mathrm{mm})$ & $(\mathrm{mm})$ & $(\mu \mathrm{l})$ \\
\hline$\# 1$ & 1.4 & 2.6 & 4.00 \\
\hline$\# 2$ & 1.3 & 2.5 & 3.31 \\
\hline$\# 3$ & 1.3 & 3.0 & 3.98 \\
\hline$\# 4$ & 1.3 & 2.5 & 3.32 \\
\hline$\# 5$ & 1.3 & 2.5 & 3.32 \\
\hline$\# 6$ & 1.4 & 2.6 & 4.00 \\
\hline$\#+$ & 1.4 & 2.9 & 4.46 \\
\hline$\# 8$ & 1.4 & 2.8 & 4.31 \\
\hline$\# 9$ & 1.4 & 2.9 & 4.46 \\
\hline$\# 10$ & 1.3 & 2.8 & 3.72 \\
\hline & & & average 3.889 \\
\hline & & & std. deviation 0.457 \\
\hline
\end{tabular}



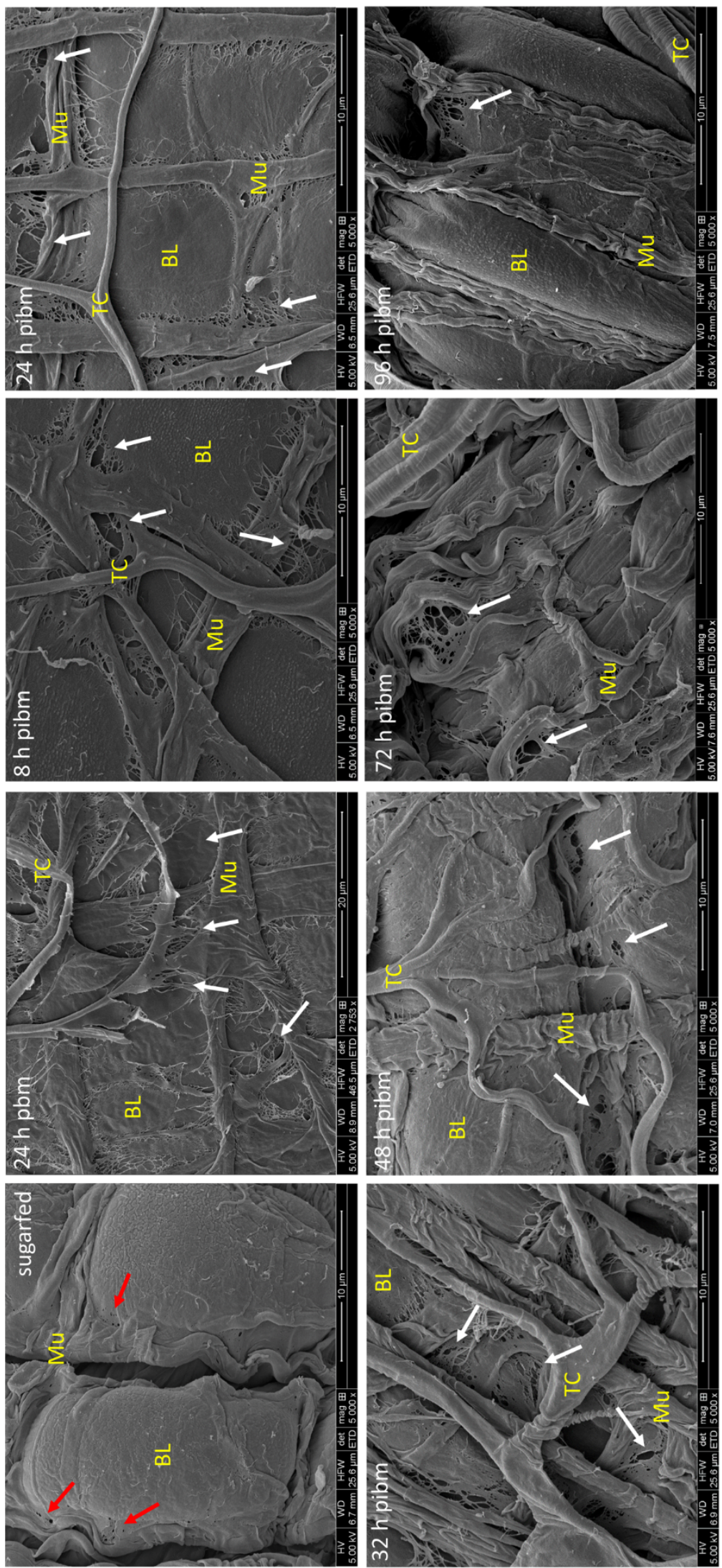
Figure 15. Ultrastructural (SEM) imaging of the midgut surface of Ae. aegypti.

SEM images of the midgut in comparison to mosquitoes that had ingested a non-infectious, artificial bloodmeal at $24 \mathrm{~h}$ post-bloodmeal $(\mathrm{pbm})$ or a CHIKV containing, artificial bloodmeal (titer in the bloodmeal: $10^{7} \mathrm{pfu} / \mathrm{mL}$ ) at $8,24,32,48,72$, and $96 \mathrm{~h}$ post-infectious bloodmeal (pibm). The midgut tissue of sugarfed females appears relaxed and deeply invaginated. Midgut-associated circular and longitudinal muscles are visible. Red arrows point to slight tissue damage occasionally visible, due to tearing of the basal lamina (BL). At $8 \mathrm{~h}$ pibm, the outer layer of the BL is torn and disrupted in close proximity to muscle tissue (exemplified by white arrows). Disruption of the outer over-stretched BL layer adjacent to muscle tissue is strongly apparent at 24 $\mathrm{pbm} / \mathrm{pibm}$ and $32 \mathrm{~h} \mathrm{pibm}$. At $48 \mathrm{~h}$ pibm, the midgut $\mathrm{BL}$ is beginning to relax and contract, torn areas of the BL near to muscles become less prominent. This trend continues; at $72-96 \mathrm{~h}$ pibm, disrupted regions of the outer BL layer are still visible although less prominent. Images were generated using a FEI Quanta 600F scanning electron microscope. All images (except: $24 \mathrm{~h} \mathrm{pbm}$ at $2753 \times$ magnification) were captured at $5000 \times$ magnification. $\mathrm{BL}=$ basal lamina; $\mathrm{Mu}=$ muscle; $\mathrm{TC}=$ tracheal cell. 
thinly stretched and its outmost layer(s) was (were) torn near to the circular and longitudinal muscles surrounding the midgut organ. Between 24 and $48 \mathrm{~h}$ pibm, $\mathrm{BL}$ overstretching and tearing near muscles was strongly apparent in all samples that had been analyzed. From $72 \mathrm{~h}$ pibm onwards, the midgut tissue became increasingly relaxed and extensively invaginated. Areas where the $B L$ was torn became less prominent, but were still visible at $96 \mathrm{~h} \mathrm{pibm}$. All of the SEM images showing midgut tissue after bloodfeeding were obtained from mosquitoes that also had been orally infected with CHIKV (with the exception of $24 \mathrm{~h} \mathrm{pbm}$ in Fig. 15). The virus titer in the bloodmeal was $\sim 10^{7} \mathrm{pfu} / \mathrm{ml}$. Although tiny globular structures that resembled virions were occasionally apparent on midgut surfaces, we were not sufficiently convinced that these structures were indeed virions. Multiple attempts to conduct immuno-gold labeling on these samples failed due to technical difficulties. To overcome this shortcoming, FIBSEM tomography was conducted by collecting and processing 108 consecutive images, each $20 \mathrm{~nm}$ apart, of virus infected midgut epithelium. As described in the next paragraph, this approach enabled us to detect and visualize $\mathrm{CHIK}$ virions within the $B L$ structure.

\section{CHIKV disseminates from the midgut within a narrow time window during bloodmeal digestion}

TEM visualization of a posterior midgut cross section shows the organization of the single cell layer. At the luminal side, the cell layer possesses microvilli for nutrient absorption followed by a zone largely comprised of ER, 

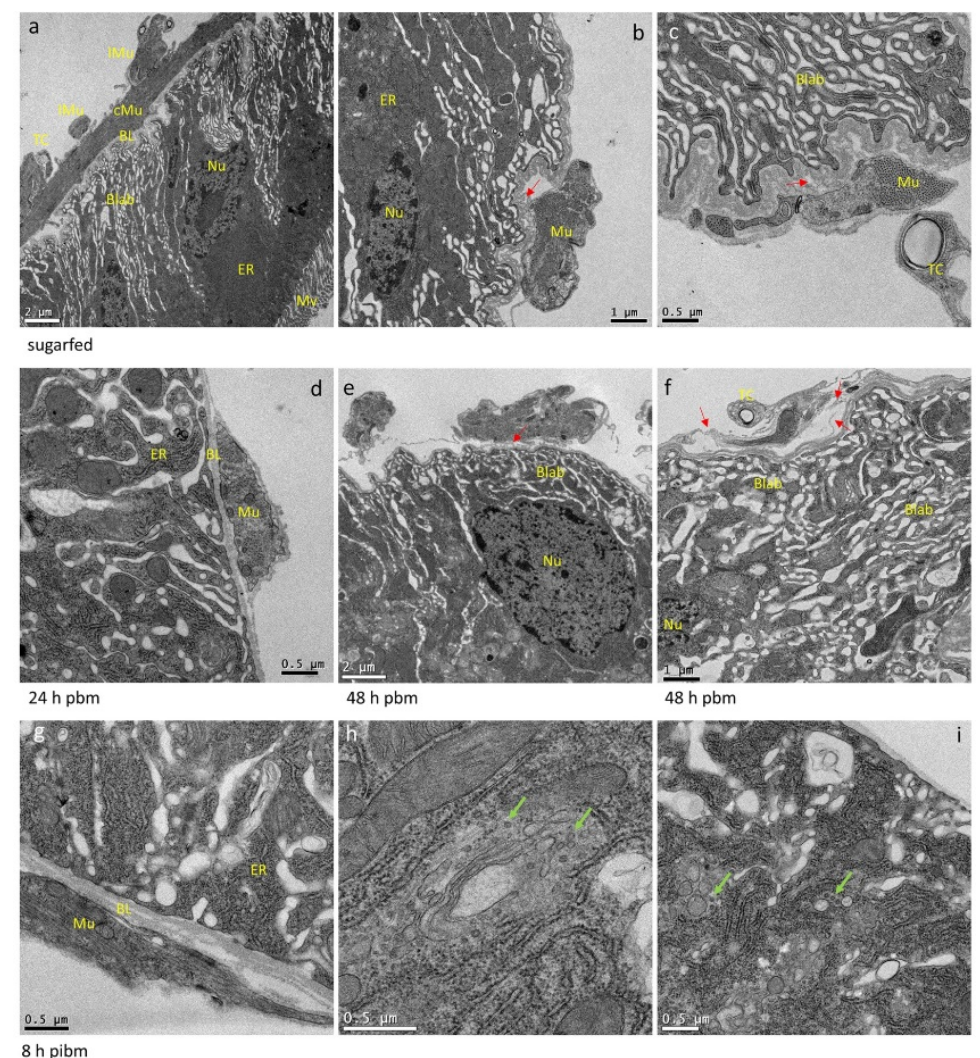

$8 \mathrm{~h} \mathrm{pibm}$
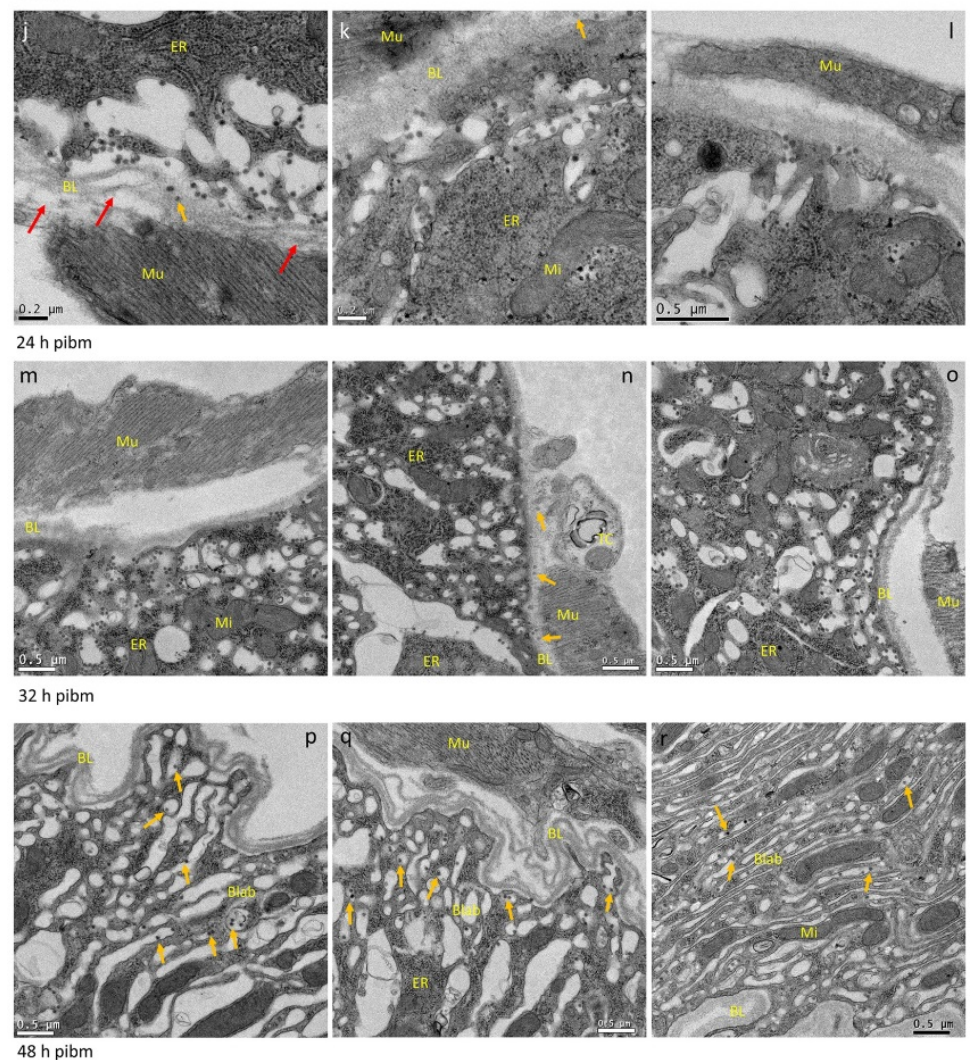
Figure 16. Ultrastructural (TEM) imaging of midgut cross-sections obtained from female Ae. aegypti, which had been sugarfed, bloodfed or orally infected with CHIKV (titer in the bloodmeal: $\left.10^{7} \mathrm{pfu} / \mathrm{ml}\right)$.

(a-c) midgut cross sections of sugarfed mosquitoes. The midgut epithelium consists of a single cell layer. Luminal microvilli of an epithelial cell are visible in (a), followed by a dense ER containing zone, the nucleus, and a prominent basal labyrinth, which stretches out until reaching the $\mathrm{BL}$ surrounding the entire organ. At the hemocoel facing side of the BL (outside the midgut), circular and longitudinal muscles, as well as tracheal cells are visible. In sugarfed midguts, the BL appears relaxed although splicing of individual strands is occasionally visible (red arrows). (d-f) The $\mathrm{BL}$ of bloodmeal containing midguts is stretched and in proximity to muscles and tracheal cells, BL strands are often severely spliced (red arrows). ( $\mathrm{g}-\mathrm{i})$ At $8 \mathrm{~h}$ post-infectious bloodmeal (pibm), mature CHIK virions close to the BL are not yet apparent. Instead, in the ER, groups of particles resembling CHIKV nucleocapsids ( $40 \mathrm{~nm}$ diameter) are visible within membranous compartments (green arrows). ( $\mathrm{j}-\mathrm{I})$ Predominantly in zones around the muscles, mature CHIK virions are concentrated within the cavities of the basal labyrinth and strongly accumulating at the $\mathrm{BL}$ at $24 \mathrm{~h}$ pibm; virions are occasionally visible within strands of the $\mathrm{BL}$ (orange arrows). In the zones next to muscles, the $B L$ is distorted as strands are severely spliced (red arrows) and/or the overall texture of the BL looks porous. (m-o) At $32 \mathrm{~h}$ pibm, the situation described for $24 \mathrm{~h}$ pibm has further developed as mature $\mathrm{CHIK}$ virions are now more frequently observed within strands of the $\mathrm{BL}$ (orange arrows) indicative of virus dissemination from the midgut. ( $p-r)$ At $48 \mathrm{~h}$ pibm, the midgut $\mathrm{BL}$ looks wavy, due to progressive bloodmeal digestion causing the midgut tissue to contract. As shown in $(p, q), B L$ strands look severely spliced, especially in proximity to muscle tissue. CHIK virions no longer accumulate in large quantities at the BL. Instead, they are dispersed and solitary within the cavities of the basal labyrinth (exemplified by orange arrows). This situation prevails at later time points, for example at 5 days pibm (see also Figure 6a,b) until the mosquito ingests a second bloodmeal. (a-r) Three different midgut samples per time point were analyzed. Images were generated using a JEOL JEM 1400 transmission electron microscope. Magnifications ranged from $2500 \times$ to $10,000 \times$. BL $=$ basal lamina; Blab $=$ basal labyrinth; $\mathrm{ER}=$ endoplasmic reticulum; $\mathrm{IMu}=$ longitudinal muscle; $\mathrm{cMu}=$ circular muscle; $\mathrm{Mu}=$ muscle; $\mathrm{Mi}=$ mitochondrion; $\mathrm{Mv}=$ microvilli; $\mathrm{Nu}=$ nucleus; $\mathrm{TC}=$ tracheal cell. 
nucleus, Golgi-apparatus, and mitochondria, which is interspersed with another zone defined as the basal labyrinth (Fig. 16a-c) (189). Virion assembly and maturation of alphaviruses take place at the plasma membrane within the basal labyrinth (250), which stretches out to the BL surrounding the midgut and separating it from the hemocoel.

In our cross-sections, the principal organization of the midgut epithelium of a sugarfed mosquito looked similar to that of bloodfed mosquitoes at 24 or $48 \mathrm{~h}$ pbm (Fig. 16a-f). However, in midguts of sugarfed mosquitoes, the BL was more relaxed and wavy in appearance, whereas in the bloodfed midguts, especially before $48 \mathrm{~h} \mathrm{pbm}$, the $\mathrm{BL}$ was stretched out. Individual measurements of $\mathrm{BL}$ thickness confirmed that in sugarfed mosquitoes the midgut BL was on average $232 \mathrm{~nm}$ thick $(N=20)$, whereas at $8 \mathrm{~h}$ pibm, the $\mathrm{BL}$ was on average only $149 \mathrm{~nm}$ thick $(N=25)$ (Fig. 17a).

Concurrent with progressive bloodmeal digestion, the average BL thickness gradually increased overtime to reach between $32 \mathrm{~h}$ and $48 \mathrm{~h}$ pibm a thickness similar to that of non-bloodfed (sugarfed) mosquitoes. Predominantly near to muscles, the BL was distorted and spliced into its individual strands as indicated by the red arrows in Fig. 16. This was observed in midguts of bloodfed and to a lesser extent also in those of sugarfed mosquitoes. At $8 \mathrm{~h}$ pibm (titer in the bloodmeal: $\sim 10^{7} \mathrm{pfu} / \mathrm{ml}$ ), which is within the range of typical human viremia titers (251), mature CHIK virions were not yet visible in the basal labyrinth. Instead, de novo assembled capsids were occasionally visible within vesicle-like 
a

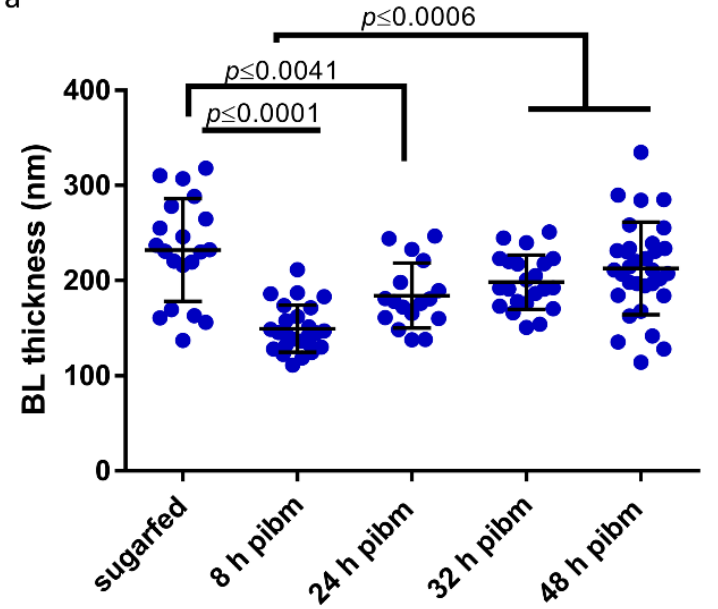

b

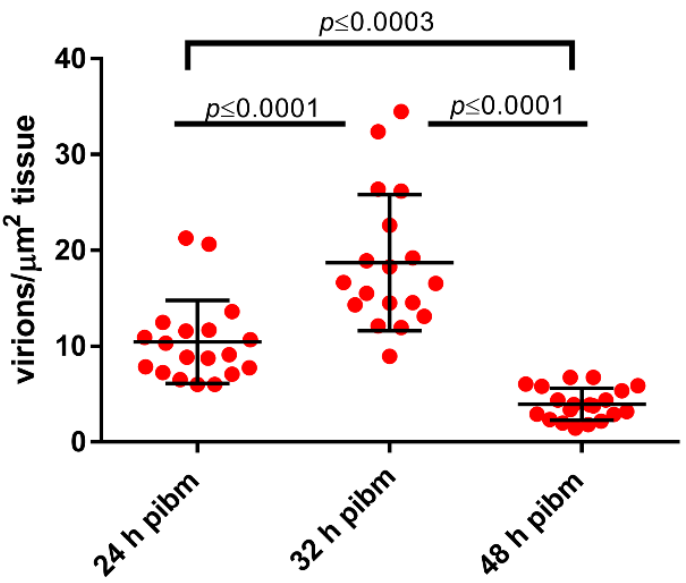

Figure 17. Basal lamina thickness and CHIK virion density at the $B L$

(a) Thickness of the midgut basal lamina (BL) of Ae. aegypti and (b) CHIK virion density in the basal labyrinth of infected midgut epithelial cells at different time points post-infectious bloodmeal (pibm). (a) Measurements were performed in those regions where the BL looked intact and was not severely spliced. $(\mathbf{a}, \mathbf{b})$ Each data point represents a single distinctive TEM image. Bars indicate mean values, Error bars represent standard deviations. Significant differences between mean values based on One-way ANOVA followed by Tukey's multiple comparisons test are shown. 
structures in the cytoplasm (Fig. 16h-i, green arrows). These virus-like structures had a diameter of around $40 \mathrm{~nm}$, resembling the size of assembled alphavirus capsids $(149,252)$ and were absent in non-infected control samples (Fig. 16a-f). Sixteen hours later (at $24 \mathrm{~h}$ pibm), however, mature virions were apparent in the basal labyrinth in close proximity to the BL (Fig. 16j-I ). A few virions were visible between strands of the $\mathrm{BL}$ (orange arrows). In general, the virus was unevenly distributed throughout the epithelium of a midgut with typically only 2-3 regions (cells) showing strong virus infection. Accordingly, the average virion density was $\sim 10$ virions $/ \mu \mathrm{m}^{2}$ (Fig. $17 \mathrm{~b}$ ). We consistently noticed that the prominent regions where virions tended to amass were those in close proximity to circular and longitudinal muscles surrounding the midgut. As mentioned and shown above, these were also the regions where the multilayered BL appeared to be spliced into individual strands (red arrows). We assume that these distorted, spliced BL regions corresponded to the torn areas of $\mathrm{BL}$ that were visible in the SEM preparations (Fig. 15).

Our FIB-SEM analysis based on serial imaging at $20 \mathrm{~nm}$ intervals confirmed that the BL formed a multi-layered dense mesh surrounding the mosquito midgut and that CHIKV was associated with the BL (Fig. 18a-g). At 24 h pibm, CHIK virions were detected within the three-dimensional space between the layers of the $B L$ when segmented and rendered as a translucent structure (Fig. 18c,e). Regions of the BL in close proximity to muscles appeared to be distorted (Fig. 18c,d,g). In general, virions within the BL were individually dispersed rather than densely clustered together. However, when the BL was 

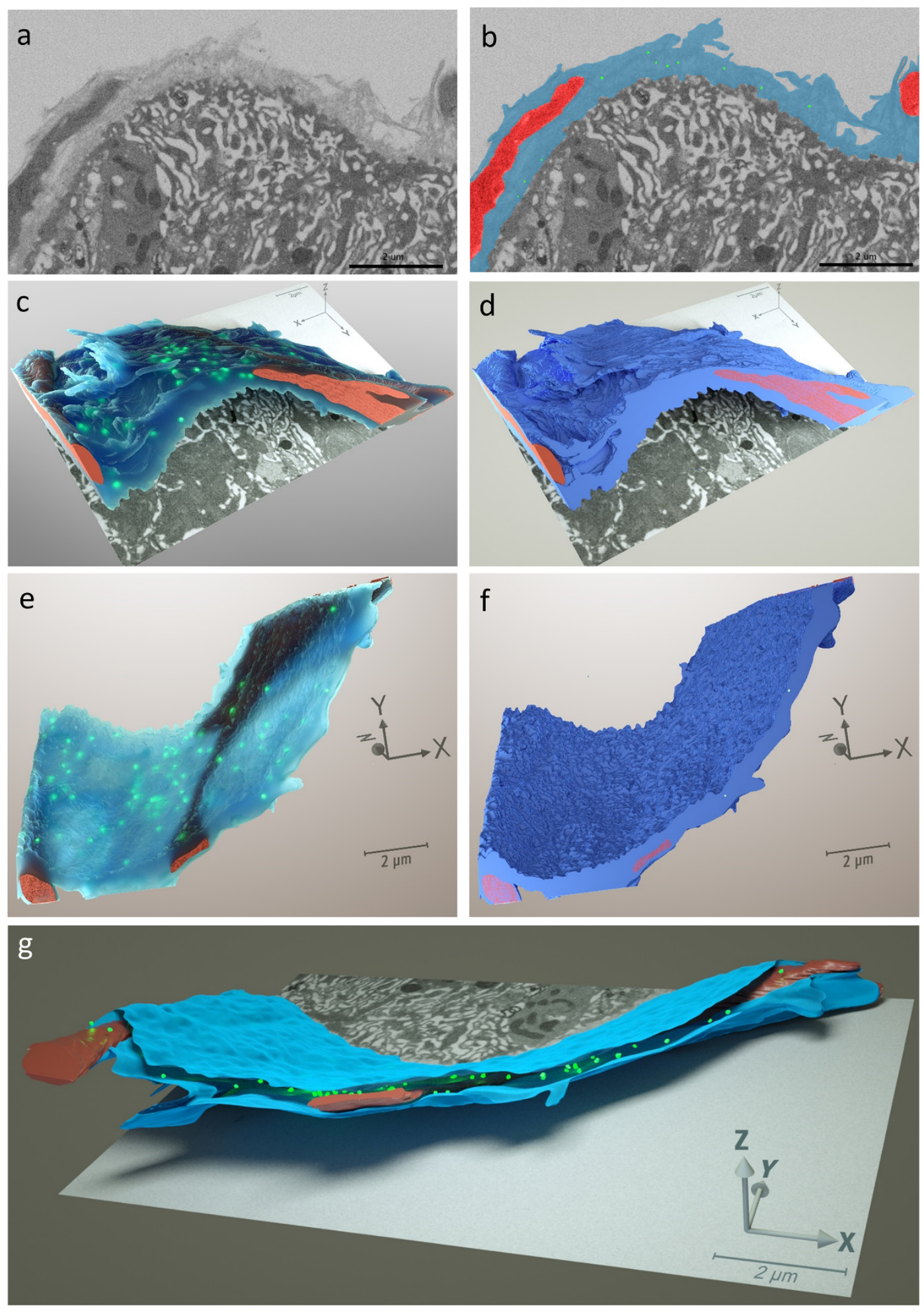
Figure 18. Three-dimensional (3-D) reconstruction from 108 stacked FIB-SEM-generated images serially sectioned at 20-nm intervals showing midgut tissue obtained from a CHIKV infected mosquito at $24 \mathrm{~h}$ post-infectious bloodmeal (pibm).

(a) Two-dimensional $(x, y)$ sample image (\# 101) out of a stack of 108 FIB-SEM images $(z=20$ $\mathrm{nm}$ ) showing image resolution before image alignment and rendering of the three-dimensional model. (b) Same image as shown in (a) with false coloration of muscles (red), BL (cyan) and $\mathrm{CHIK}$ virions (green). (c) Global, translucent view of the sample section that had been analyzed by FIB-SEM following three-dimensional modeling. Blue coloration: Midgut BL (note the disrupted outer layer(s) of the $\mathrm{BL}$ ); red coloration: Muscle tissue surrounding the midgut. $\mathrm{CHIK}$ virions (green) are clearly visible being embedded within the multi-layered BL. The basal labyrinth of the epithelial cell is visible along the $x$ and $y$ axes. (d) Opaque representation of the same sample shown in panel c. CHIK virions are absent on the BL surface facing the hemocoel. (e) Another 3$D$ view of the translucent midgut $B L$ structure showing the presence of virions within the $B L$ layers. (f) Those virions are no longer visible in the opaque (surface) representation of the same sample. (g) Frontal view presenting the innermost (top) and outermost (bottom) layers of the midgut $\mathrm{BL}$ with individual $\mathrm{CHIKV}$ virions being located between those BL layers. All image reconstructions were initially generated using the Amira software suite v. 5.3.1. Images were then further refined in Maxon Cinema 4D R19 using the software OTOY Octane Render V3. Bars in $(\mathbf{a}-\mathbf{g})$ represent a length of $2 \mu \mathrm{m}$. 
presented as an opaque structure, virions were not visible on the BL surface, but within the inner- and outermost layers of the BL (Fig. 18d,f,g). It may be possible that any virions located on the BL surface have been washed away during FIBSEM sample processing.

At $32 \mathrm{~h}$ pibm, virion accumulation at the $\mathrm{BL}$ was further enhanced (average virion density: $\sim 19$ virions/ $\mu \mathrm{m}^{2}$; Fig. $17 \mathrm{~b}$ ), with CHIK virions in the TEM preparations now being more frequently visible between strands of the BL (Fig. 16m-o, orange arrows). Importantly, we did not observe CHIKV infecting the midgut associated muscles at any time point during the $48 \mathrm{~h}$ observation period. We also did not detect virions in the tracheal tissue surrounding the midgut in 30 different samples that were analyzed, with a single exception at $40 \mathrm{~h}$ pibm (Fig. 19a,b, yellow arrows). At $48 \mathrm{~h}$ pibm, the midgut epithelium became less stretched as indicated by the wavy shape of the BL (Fig. 16p-r). Furthermore, virions no longer amassed towards the BL. Instead, they became more dispersed inside the basal labyrinth (average virion density: $\sim 4$ virions $/ \mu \mathrm{m}^{2}$; Fig. $17 \mathrm{~b}$ ) as exemplified by orange arrows in Fig. 16p-r. At later time points, i.e., 5 and 7 days pibm, this pattern still prevailed (Fig. 20a,b) (189). Based on these observations, we propose that there is a relatively narrow time span from approximately $24 \mathrm{~h}$ to less than $48 \mathrm{~h}$ pibm, during which $\mathrm{CHIKV}$ has the opportunity to exit the midgut epithelium across the BL to infect secondary tissues. 

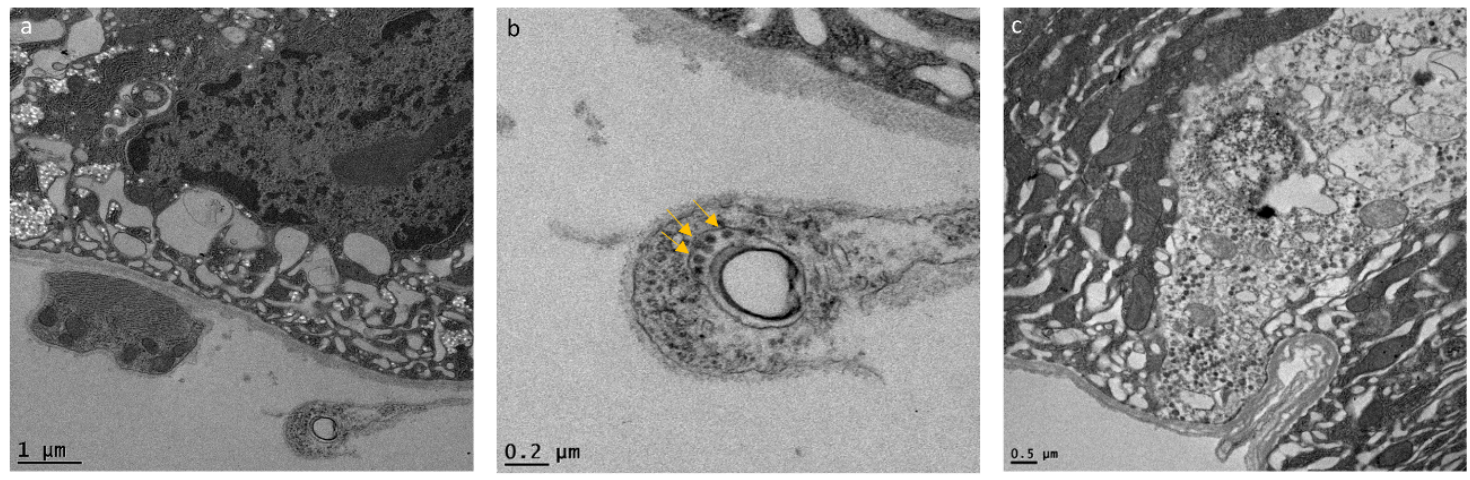

Figure 19. Rare event of an infected trachea observed with TEM

(a, b): Infection of tracheal tissue with CHIKV at $40 \mathrm{~h}$ post-infectious bloodmeal (pibm). (a) The nearby region of the midgut epithelium does not appear to be virus infected. (b) Higher magnification of the tracheal cell cross section of (a) showing the presence of virions (yellow arrows). (c) A degenerating cell with a vesicular appearing nucleus and a reduced cytoplasmic density. The structure of the ER is no longer visible, however the BL is intact. 

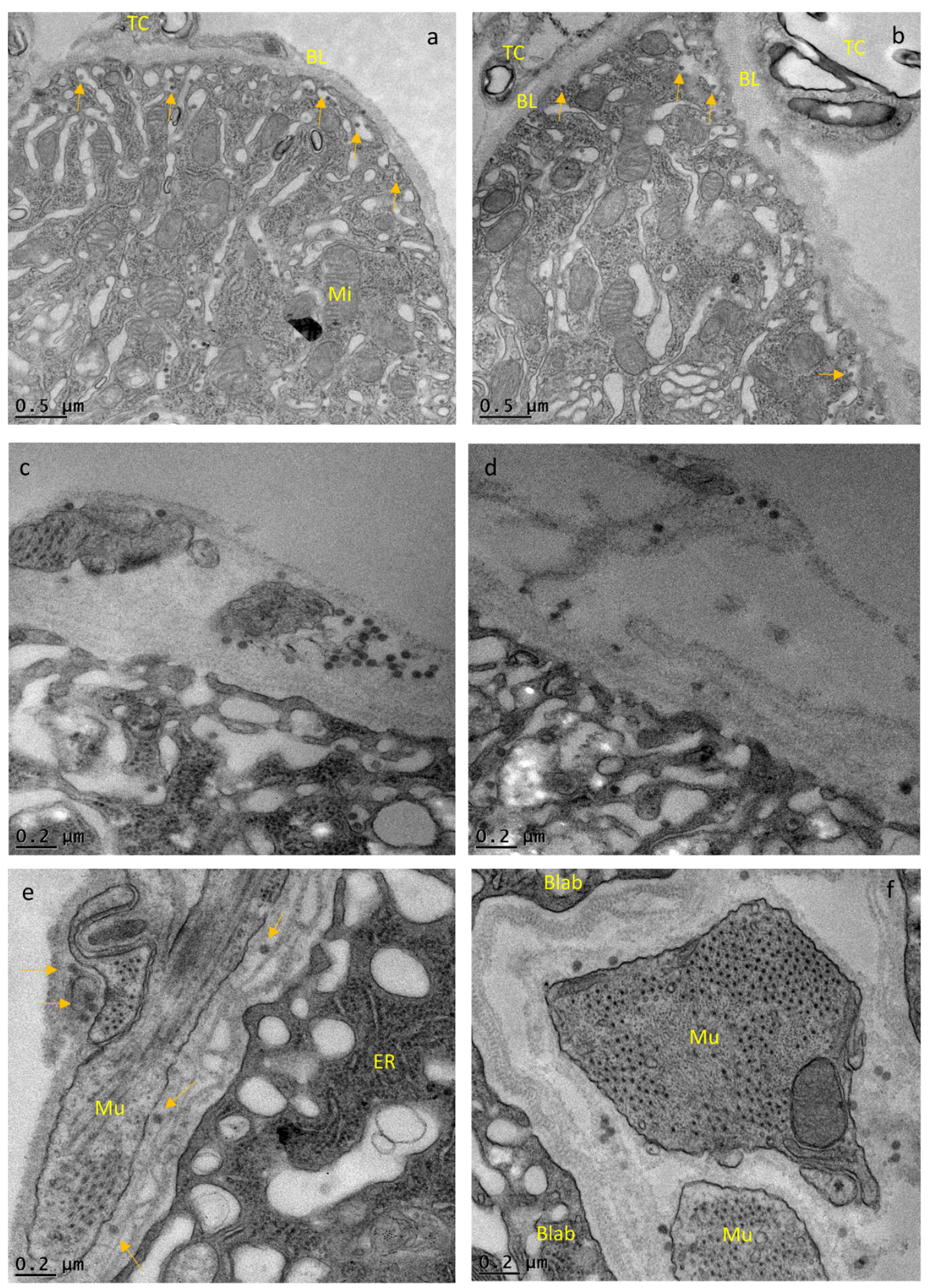
Figure 20. Ultrastructural (TEM) examination to assess how multiple bloodmeals affect CHIKV dissemination from the midgut of Ae. aegypti.

Midgut cross-sections of females that had received either a single CHIKV containing bloodmeal (titer in the bloodmeal: $10^{7} \mathrm{pfu} / \mathrm{ml}$ ) or an initial bloodmeal containing CHIKV followed by a noninfectious ( $\mathrm{NI})$ bloodmeal were analyzed. $(\mathbf{a}, \mathbf{b})$ Mature $\mathrm{CHIK}$ virions are dispersed throughout the cavities of the basal labyrinth of the infected midgut epithelial cell at 5 days post-initial, infectious bloodmeal. Occasionally, single virions are visible close to the $B L$ (orange arrows). (c,d) At $24 \mathrm{~h}$ post-second ( $\mathrm{NI}$ ) bloodmeal (144 h post-initial CHIKV containing bloodmeal), virions are found within strands of the $B L$ and attached to the side of the $B L$ facing the hemocoel suggesting that these virions have traversed the BL. Occasionally, virions are also found to be attached to $B L$ fragments that are largely detached from the main BL surrounding the midgut organ. $(\mathbf{e}, \mathbf{f})$ At $32 \mathrm{~h}$ post-second $(\mathrm{NI})$ bloodmeal (152 $\mathrm{h}$ post-initial CHIKV containing bloodmeal), virions are still present between strands of the $B L$ or associated with the hemocoel facing side of the $B L$, predominantly in proximity to the muscle tissue (orange arrows). (a-f) Images were generated using a JEOL JEM 1400 transmission electron microscope. Magnifications ranged from $2500 \times$ to $25,000 \times . \mathrm{BL}=$ basal lamina; $\mathrm{Blab}=$ basal labyrinth; $\mathrm{ER}=$ endoplasmic reticulum; $\mathrm{Mu}=$ muscle; $\mathrm{Mi}=$ mitochondrion; $\mathrm{Nu}=$ nucleus; $\mathrm{TC}=$ tracheal cell. 


\section{Repeated bloodmeals enhance virion dissemination from the midgut epithelium}

We exposed mosquitoes that had been orally infected with CHIKV via an initial bloodmeal to a second, non-infectious ( $\mathrm{NI})$ bloodmeal (at 5 days post-initial bloodmeal) to see whether the midgut dissemination process we had observed after ingestion of a single bloodmeal was repeated during a subsequent bloodmeal digestion. At 5 days post-single infectious bloodmeal, virions were dispersed throughout the cavities of the basal labyrinth in proximity to the BL. At this time point virions did not amass at the BL (Fig. 20a,b, orange arrows).

However, at 24 and $32 \mathrm{~h}$ post second NI bloodmeal, CHIK virions were repeatedly observed outside the midgut epithelium in association with strands of the BL (Fig. 20c-f). This differs from the observations made in midguts of those mosquitoes, which had received only a single, infectious bloodmeal. As described above, in singly bloodfed females, CHIK virions were observed between strands of the $\mathrm{BL}$, but not outside the $\mathrm{BL}$.

\section{Quantification of alphavirus RNA in infected midguts and carcasses over time indicates that virus dissemination from the midgut occurs within $48 \mathrm{~h}$ pibm}

When comparing the viral replication of CHIKV (strain 37997) with that of a related alphavirus, MAYV (strain TRVL 4675), in midguts and carcasses of HWE mosquitoes by Taqman qRT-PCR, similar patterns became apparent. In midgut tissue, quantities of plus- and minus-strand viral RNAs of both viruses 
CHIKV plus-strand

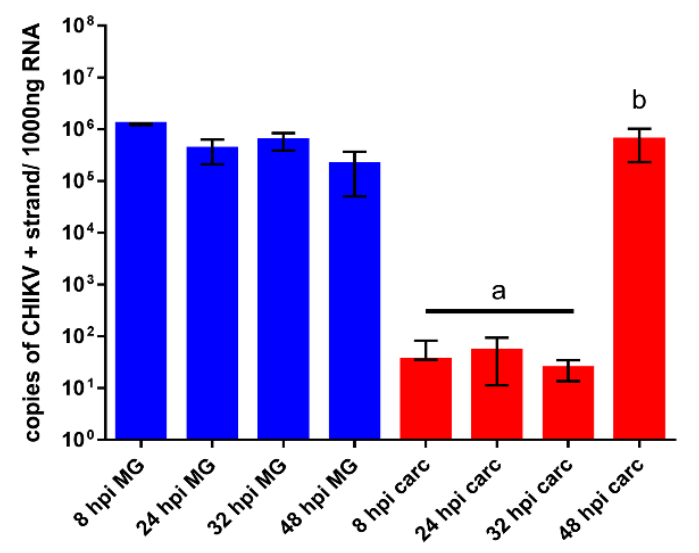

MAYV plus-strand

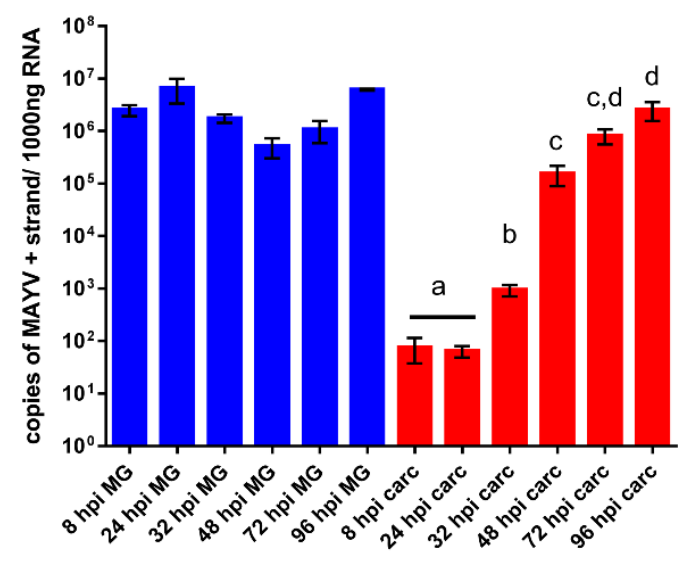

CHIKV minus-strand

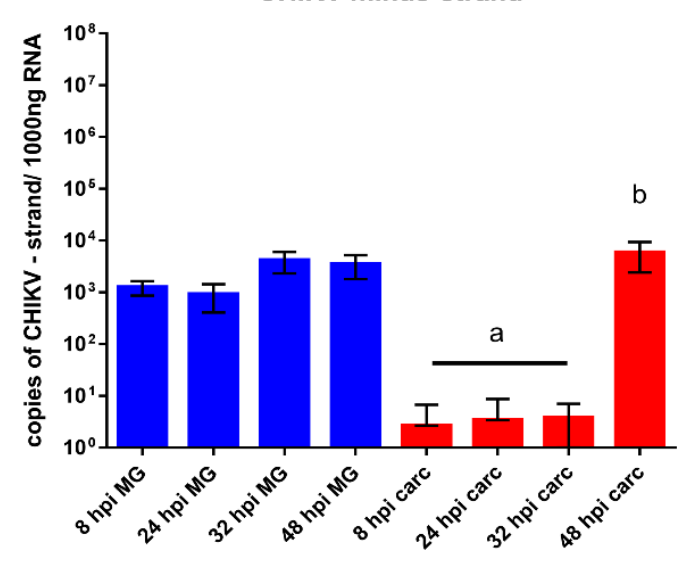

MAYV minus-strand

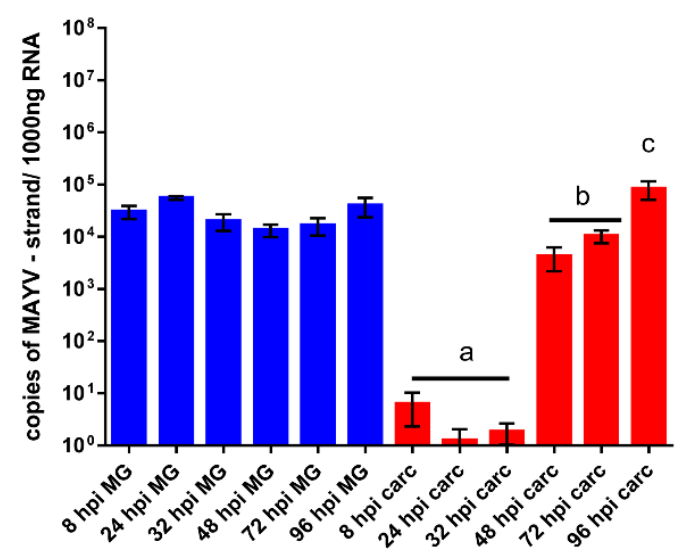

Figure 21. Quantitative detection of plus- and minus-strand RNAs of CHIKV and Mayaro virus (MAYV) via Taqman qRT-PCR assays.

RNA detection and quantification in midguts (blue) and carcasses (red) of Ae. aegypti at various time points post-infectious bloodmeal. Each sample consisted of total RNA extracted from 10 midguts or three carcasses of CHIKV or MAYV challenged mosquitoes. For each time point the mean value of three biological replicates (=three samples) is shown. Error bars represent standard deviations. Different letters above bars (shown only for carcass values) indicate significant differences of mean values based on One-way ANOVA followed by Tukey's multiple comparisons test $(p<0.05)$. 
reached a plateau by $8 \mathrm{~h}$ pibm (Fig. 21). The values of viral plus-strand RNA copy number equivalents were between $10^{5}$ and $10^{6}$ for $\mathrm{CHIKV}$ and $10^{6}$ to $10^{7}$ for MAYV. At 8-24 h pibm, plus-strand RNA detected in midgut tissue likely includes the viral RNA of the input virus from the bloodmeal. Quantities of minus-strand RNAs, indicative of active viral replication, were 2-3 logs lower in midgut tissue than those of viral plus-strand RNA molecules. This observation is in line with the alphavirus replication strategy described above, in which viral plus-strand RNA synthesis continues throughout the infection cycle while minus-strand RNA synthesis has already been shut off $(68,212)$. In carcass tissue, plus- and minusstrand RNA copy number equivalents were only minimally detectable $(<100$ copies) for both viruses until $48 \mathrm{~h}$ pibm, when minus-strand RNA copy number equivalents significantly increased. This significant increase in minus-strand RNA copy number equivalents indicates productive virus infection of secondary tissues outside the midgut. We assume that there is a time gap between virus dissemination from the midgut and actual productive (measurable) infection of secondary tissues. Thus, our Taqman qRT-PCR results support our ultrastructural observations (Fig. 16, Fig. 17, and Fig._21) suggesting that CHIKV dissemination from the midgut occurred within $48 \mathrm{~h}$ pibm, before virions inside the epithelial cells stopped congregating towards the midgut BL.

\section{Discussion}

In a previous study two Ae. aegypti laboratory strains, HWE and ORL, were analyzed for their vector competence for CHIKV (strain: 37997) (14). The 
data revealed that as early as $1-2$ days post-oral acquisition, the virus already produced high titers in midgut tissue and disseminated from the midgut to secondary tissues. By $48 \mathrm{~h}$ pibm, CHIKV was detectable in salivary glands and in saliva. These characteristics, high virus titers and fast dissemination, made CHIKV 37997 an attractive model for observing its dissemination from the midgut in a time course study at the ultrastructural level. Our artificial feeding system may not exactly reflect the amount of virus intake as might be observed with viremic bloodmeals obtained from naturally CHIKV infected hosts. Viremic bloodmeals may be smaller than artificial bloodmeals obtained via membrane feeding in a controlled environment. Furthermore, mosquitoes, such as Ae. aegypti, tend to ingest multiple viremic and/or non-viremic bloodmeals from the human host, which likely affect midgut infection and dissemination dynamics of an arbovirus $(253,254)$. However, our artificial bloodmeal procedure has the advantages of standardization and reproducibility. Earlier, we revealed that a single one-week old female ingests $\sim 20,000$ pfu CHIKV 37997 from an artificial bloodmeal containing $\sim 10^{7} \mathrm{pfu} / \mathrm{ml}$ virus (14), a titer that is within the range of viremic bloodmeals (251). Thus, the bloodmeal intake of a fully engorged female would amount to a volume of $\sim 2 \mu$ l. All observations made in this study are based on these parameters. Our SEM analysis revealed that bloodmeal ingestion leads to midgut tissue (over)stretching and partial BL disruption (Fig. 22 a).

Importantly, the disruption of the BL was predominantly observed alongside muscles surrounding the midgut as the bloodmeal containing tissue 
a sugarfed bloodfed

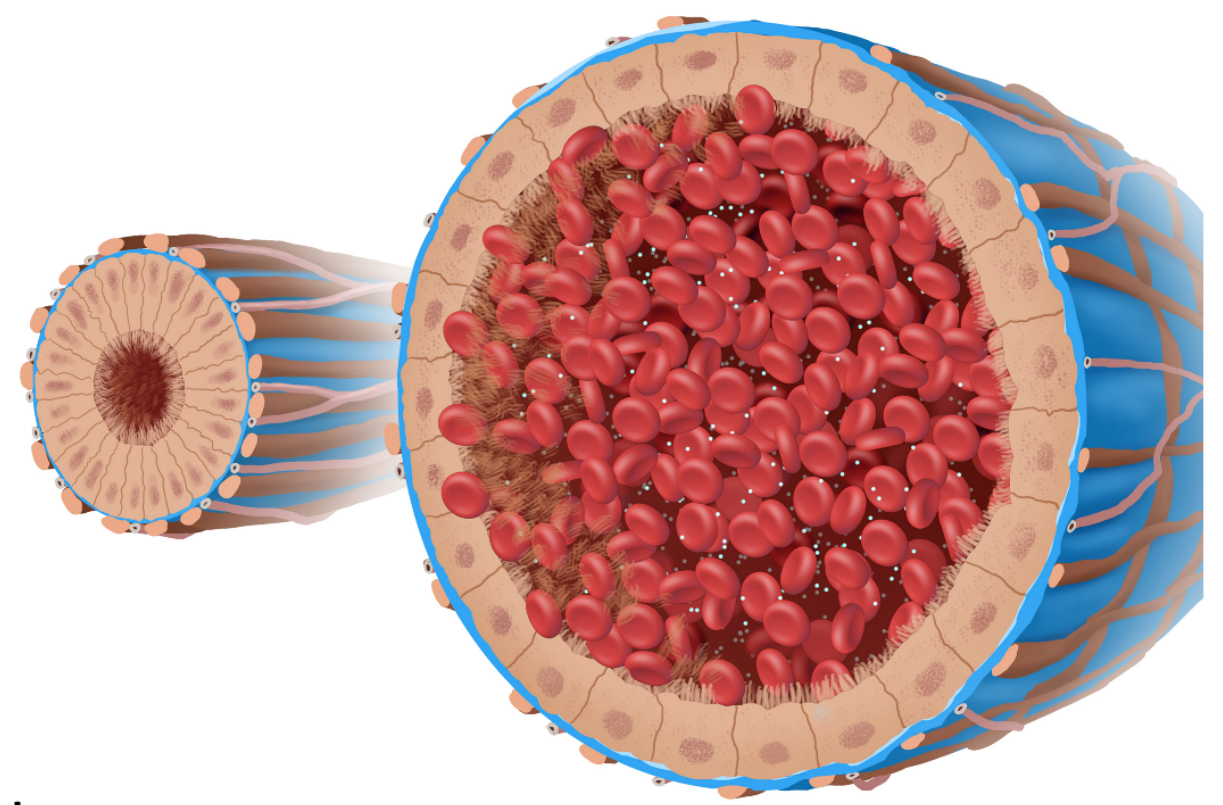

b

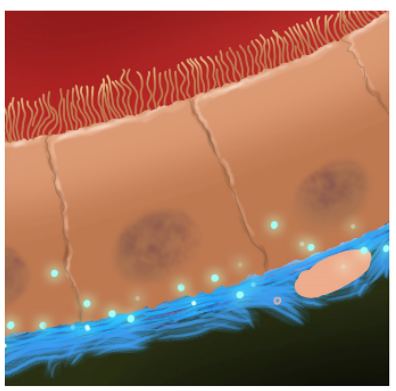

$24 \mathrm{~h}$ pibm

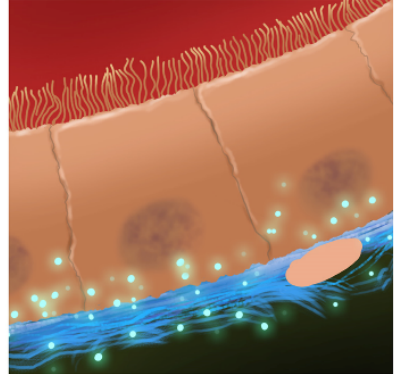

$32 \mathrm{~h}$ pibm

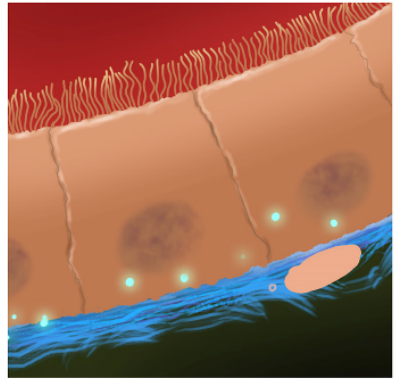

$48 \mathrm{~h}$ pibm

Figure 22. Schematic representation of CHIKV dissemination from the midgut of Ae. aegypti.

(a) Midgut cross section of a sugarfed female (left) in comparison to a midgut of a female that has ingested a CHIKV-containing bloodmeal (right; virions are shown in green). Note the $\sim 20 \times$ increase in diameter of the bloodmeal containing midgut (see also Fig. 14). Also: The midgut epithelial cells of the sugarfed mosquito have a columnar shape whereas those of the bloodfed female look flattened. (b) Dissemination pattern of CHIKV (green) across the midgut BL (blue) at 24,32 , and $48 \mathrm{~h}$ post-infectious bloodmeal (pibm) according to the observations shown in Fig. 16 indicating that within a time span of $48 \mathrm{~h}$ pibm the virus is exiting the midgut. The figure was created in Adobe Photoshop CC 2018 (Adobe Systems, San Jose, CA, USA). 
was bulging out between those muscles. During progressive digestion of the bloodmeal, the midgut tissue subsequently relaxed and the damage to the $\mathrm{BL}$ was less pronounced although ruptures to the BL still prevailed at 72 or $96 \mathrm{~h}$ pibm, when the bloodmeal had been completely digested. The SEM samples included bloodfed midguts from CHIKV infected mosquitoes, however, we were unable to clearly identify midgut associated virions in those samples based on their size, shape, and quantity. We chose FIB-SEM (244-248) as an alternative technique and this approach enabled us for the first time to visualize virions in the three-dimensional space of a mosquito tissue. As CHIKV infection foci were irregularly distributed in the complex midgut tissue, it was initially challenging to select a suitable region of the tissue (indicated by a high abundance of virions) for FIB-SEM preparation. To overcome this complication, we prescreened a sample via TEM to identify a landmark, such as virion abundance, in a location of the sample before orienting the FIB-SEM sample for imaging. The preselected (landmarked) tissue was then mounted with the block face perpendicular to the stub surface instead of mounting it parallel to the surface of an SEM stub as a typical protocol would recommend $(185,186)$. This provided several advantages, including a proper orientation of the sample to the SEM beam for final data collection, a reduction in the time needed to properly expose the surface of interest using ion milling, and an easy identification of the region-of-interest using the landmark(s) established with TEM.

Our TEM images unequivocally showed the presence of $\mathrm{CHIK}$ virions in the midgut epithelium without any need for antibody labeling. Whereas at $8 \mathrm{~h}$ 
pibm, assembled virions were not yet visible, they became apparent in large quantities in the midgut labyrinth and in association with the BL between 24 and $32 \mathrm{~h}$ pibm, indicative of dissemination from the midgut (Fig. 22b). A similar time window of virion accumulation towards the $B L$ had been earlier reported for Western equine encephalitis virus (WEEV; Togaviridae, Alphavirus) $(149,214)$. Furthermore, we noticed that preferred zones of virion congregation towards the BL occurred in close proximity to muscles although muscle tissue itself did not become infected with the virus during the time period of dissemination. Our TEM images also showed that in close proximity to muscles, the BL strands often looked severely spliced and distorted corresponding to the zones of disrupted BL seen in the SEM and FIB-SEM images. However, we did not find any indication that the $\mathrm{BL}$ developed visible, distinct gaps through which virions could move. Instead, the movement through the $\mathrm{BL}$ resembled a migration/penetration-like process through the overall stretched-out $B L$ and this process might have been be assisted by extracellular matrix associated proteinases, such as MMPs, as suggested earlier $(150,243)$. Our observations are partially in agreement with those made by Romoser and colleagues $(144,151)$ regarding the dissemination of RVFV from the midgut of Ae. taeniorhynchus. The authors reported that the BL had a modified, "porous, spongy" structure in close proximity to midgutassociated muscles, which appeared to be permissive for virions whereas the intact (non-modified) BL was not. Further, the authors suggested that arboviruses, such as RVFV, would penetrate the modified $B L$ to infect the tracheo-muscular complex as a conduit for dissemination from the midgut. In our 
studies, we did not reveal that CHIKV would infect the tracheo-muscular complex as an essential step in the dissemination process from the midgut. Instead, our ultrastructural observations based on TEM and FIB-SEM suggest that within 24$48 \mathrm{~h}$ pibm, CHIK virions directly traverse the $\mathrm{BL}$ in order to infect secondary tissues outside the midgut (Fig. 22b). Accordingly, our Taqman qRT-PCR assays showed significantly increased replication of both CHIKV and MAYV in tissues outside the midgut at $48 \mathrm{~h}$ pibm. This similar timing for both viruses is remarkable, since the 37997 strain of CHIKV is a highly-passaged, laboratory adapted strain, whereas the TRVL 4675 (type) strain of MAYV from 1954 has a low passage history $(14,73)$.

The relatively short time span between the first appearance of virus-like structures in the ER of the midgut epithelial cells and that of mature virions close to the $B L$ suggests that $\mathrm{CHIKV}$ requires only a single replication cycle in the midgut epithelium before being able to disseminate to secondary tissues during bloodmeal digestion. However, following initial infection, virion production in the midgut epithelium continues and is independent of virus dissemination as shown by the fact that infection foci in the midgut tissue do increase over time $(14,240)$. We previously described that there was only a weak correlation between $\mathrm{CHIKV}$ titers in the midgut and the virus' ability to disseminate from the midgut (14). Based on our observations, we speculate that not every mature virion localized in the basal labyrinth might escape the midgut when the $B L$ is permissive during bloodmeal digestion. Those virions "left behind" may get another opportunity for dissemination during a subsequent bloodmeal without the need of an additional 
replication step. When another $\mathrm{NI}$ bloodmeal had been ingested by the CHIKVinfected female at $120 \mathrm{~h}$ post-initial infectious bloodmeal, virions again strongly congregated at the stretched BL (at 24-32 h post second NI bloodmeal). Furthermore, virions were associated with the $B L$ at its hemocoel facing side suggesting that these virions had actually traversed the BL. As pointed out above, it is possible that $\mathrm{CHIK}$ virions that remained within the basal labyrinth after the initial bloodmeal digestion are those that predominantly disseminated from the midgut during second bloodmeal digestion. Further, we speculate that during the $\mathrm{BL}$ rebuilding process after initial bloodmeal digestion, spliced fragments of the $B L$ could have occasionally been shed from the remaining $B L$ and in some instances, virions could have been attached to these shed BL fragments.

Immunohistochemical and ultrastructural studies have previously shown that the tracheal system of the mosquito can be infected with a range of arboviruses, including CHIKV $(14,144,148,149,151,152,235,236)$. Furthermore, several studies suggested that the tracheal system acts as a conduit for virus dissemination from the midgut $(144,151,235)$. Salazar and colleagues $(235)$ reported dengue 2 virus (Flaviviridae) infection of midgut associated tracheae as soon as 2 days pibm. At that time point the virus was also detectable in the mosquito carcass. Thus, it cannot be ruled out that other tissues outside the midgut were already infected by the disseminated virus before it infected the midgut associated tracheae. Dong and colleagues (14) showed that CHIKV was already detectable outside the midgut before the virus was located in tracheal 
tissue, supporting the idea of tracheal cells becoming infected after virus dissemination from the midgut. After analyzing approximately 30 different TEM samples of various time points post-CHIKV infection, we detected only a single incidence where virions were associated with a tracheal cell (Fig. 19a,b). Based on the singular observation here and our earlier confocal microscopy analysis (14), we do not consider this tissue to play a critical role in the midgut dissemination process of CHIKV.

The role of apoptosis in viral midgut escape has not been clearly revealed so far and therefore remains elusive (255-258). Previously, we have shown that bloodmeal ingestion can trigger apoptosis in midgut epithelial cells although the presence of $\mathrm{CHIKV}$ in the bloodmeal did not have any measurable effect on the apoptotic response in midgut tissue (14). In this study, we did not find any evidence that CHIKV is causing any obvious pathology in the epithelial cells of the mosquito midgut. Occasionally, we detected a degenerating cell suggestively undergoing apoptosis without any virus being present (224), (Fig. 19c).

Furthermore, the BL surrounding the degenerating cell looked intact, allowing the conclusion that an apoptotic response in a cell may not necessarily affect the integrity of the surrounding BL. This in turn would not create any benefit for CHIKV regarding its dissemination from the midgut. This is in contrast to earlier studies with Eastern equine encephalitis virus and WEEV in $\mathrm{Cu}$. melanura and $C x$. tarsalis, wherein severe virus-associated pathology was observed in infected midgut tissue $(224,225)$. Perhaps these observations resembled extreme situations based on particular virus strain-mosquito strain 
combinations, as we did not observe such severe responses associated with CHIKV infection of Ae. aegypti.

In summary, our study suggests that following a single replication cycle in the midgut epithelium, $\mathrm{CHIK}$ virions disseminate during bloodmeal digestion from the midgut epithelium by traversing the (over-) stretched $B L$ surrounding the midgut. The virus thereby is not infecting the muscle tissue and does not use the tracheal cell system to disseminate from the midgut. An important point that requires further investigation is the underlying mechanism causing the $B L$ to become permissive for the virus. Recent research work suggested that there may be an enzymatic BL modification/remodeling process involved that would include the activity of extracellular proteases $(150,243)$. Furthermore, it needs to be investigated whether other arboviruses, such as members of the Flaviviridae or Bunyaviridae, utilize the same midgut escape strategy as has been observed for CHIKV in this study. 


\section{CHAPTER V}

\section{Infection Pattern of Mayaro Virus in Aedes aegypti and Transmission Potential of the Virus in Mixed Infections with Chikungunya Virus}

This chapter has been submitted to the Journal of Medical Entomology

Asher M. Kantor ${ }^{1}$, Jingyi Lin ${ }^{1}$, Allen Wang ${ }^{2}$, Dana C. Thompson ${ }^{1}$, Alexander W.E. Franz ${ }^{1}$

AMK, and AWEF conceived and designed the experiments; AMK, JL, AW, and DCT performed the experiments; AMK, and AWEF analyzed the data, wrote, and edited the paper.

${ }^{1}$ Department of Veterinary Pathobiology, University of Missouri, Columbia, MO 65211, United States of America

${ }^{2}$ Department of Biological Sciences, University of Missouri, Columbia, MO 65211, United States of America 


\section{Abstract}

Recently, Mayaro virus (MAYV; Alphavirus; Togaviridae) has drawn increasing attention as another arthropod (mosquito)-borne virus, which may have the potential to cause major disease outbreaks among the human populations of the Western Hemisphere. So far, the virus is circulating in sylvatic cycles in the tropical regions of Central and South America. Although forestdwelling mosquitoes seem to be the major vectors for MAYV, it has been shown before that MAYV can be acquired and potentially transmitted by Aedes aegypti and Ae. albopictus. Here we compare the infection and transmission efficiencies of two MAYV strains, IQT 4235 and TRVL 4675, in two Ae. aegypti strains, HWE and ORL, showing that the TRVL 4675 strain (TRVL) was less efficiently acquired and transmitted by both mosquito strains than MAYV IQT 4235 (IQT). Based on the full-length nucleotide sequences of the two viral genomes, we show that the TRVL prototype strain of MAYV is phylogenetically ancestral and more distantly related to the IQT strain. The TRVL strain was efficiently acquired by wild-type Ae. albopictus from Columbia, Missouri and caused productive infections in those mosquitoes. Considering possible scenarios in which natural MAYV transmission cycles may overlap with those of chikungunya virus (CHIKV; Alphavirus; Togaviridae), we assessed the effects of mixed infections of the two viruses in mosquitoes based on co-infection or superinfection. Whereas coinfection had no measurable effect on the transmission potential of either virus, we observed superinfection exclusion for CHIKV in MAYV infected mosquitoes but not for MAYV in CHIKV infected mosquitoes. 


\section{Introduction}

Mayaro virus (MAYV) is an alphavirus (Togaviridae; Alphavirus) of the Semliki Forest virus antigenic complex, which was originally isolated in 1954 from the serum of a forest worker living in Mayaro County of Trinidad Island (73,259261). The virus isolate was originally designated Tr4675 and is now referred to as the MAYV prototype strain, TRVL 4675. Like other alphaviruses, MAYV is mosquito-borne. Forest- and aquatic plant dwelling mosquitoes such as Mansonia venezuelensis, Haemagogus janthinomys, Sabethes, spp., and Culex spp. have been reported as potential sylvan vectors $(261,262)$. Non-human primates such as marmosets (Calithrix argentata) are considered to be the primary reservoir for the virus $(30,262)$. MAYV has been also detected in a migrating bird (263) and in several equids in the Pantanal region, Brazil as well as in anteaters, armadillos, and opossums. Furthermore, certain rodents in French Guiana have been found seropositive for the virus $(264,265)$. MAYV represents an interesting phenomenon as it is, based on its geographic origin, considered to be a New World alphavirus with biological characteristics typical for Old World alphaviruses. MAYV infections have been known for decades among people living in tropical, rural areas of South and Central America predominantly in the vicinity of rain forest areas. The virus causes disease symptoms in humans similar to chikungunya virus (CHIKV, Togaviridae; Alphavirus) including febrile illness, rash, and (occasionally long-lasting) arthralgia (266). Like other alphaviruses, MAYV has a 12 kb single-stranded positive sense RNA genome, encoding nine genes encoding four nonstructural proteins (nsP1, nsP2, nsP3, 
nsP4) and five structural proteins (C, E3, E2, 6k/TF, E1) (62,267). MAYV strains typically exhibit a relatively narrow genetic divergence of up to $17 \%$ at the nucleotide level clustering them into three distinct genotypes named $L$ (limited), $N$ (new), and $D$ (widely dispersed). Genotype $L$ is restricted to isolates from (Belterra) Brazil, $\mathrm{N}$ to an isolate from Peru, and $\mathrm{D}$ containing isolates of wide geographical distribution $(249,267)$. In 2015 , a case has been reported in Haiti, which is the first time in the Antilles (75). This virus isolate represents a recombinant between a $D$ and an $L$ genotype virus (62). Authors of these studies also speculated whether MAYV could have the potential to become the next mosquito-borne virus, following CHIKV in 2013 and Zika virus (ZIKV; Flaviviridae; Flavivirus) in 2015, to cause large scale disease outbreaks among human populations of the Americas and the Caribbean $(62,75,249)$. A critical aspect facilitating a switch from a sylvatic to an urban arboviral disease cycle is the abundant availability of urban vectors, which would be highly susceptible for the sylvan arbovirus. Ae. aegypti and Ae. albopictus are two mosquito species that are typically involved in urban arboviral transmission cycles. Previously, an Ae. albopictus population from Brazil has been shown to be susceptible to MAYV TRVL 4675 (268). Although the Brazilian Ae. albopictus mosquitoes were considered to be relatively refractory to the virus, it was suggested that these mosquitoes still had the potential to act as secondary or bridging vectors expanding typical transmission cycles of MAYV. Furthermore, an Ae. aegypti population from Peru has been shown to be susceptible to MAYV IQT 4235 (32). The IQT 4235 strain has been isolated in 1997 from Ma. venezuelensis in the 
Loreto region of Peru (267). A recent publication describes the infection and transmission potentials of Ae. albopictus and Ae. aegypti strains from Miami and Jacksonville, Florida, USA for MAYV TRVL 4675. In their study, Ae. albopictus had a significantly higher rate of susceptibility (midgut infection) to orally acquired MAYV TRVL and produced a significantly higher number of saliva samples containing the virus than Ae. aegypti (269). Very recently, we compared the midgut dissemination efficiencies between CHIKV 37997 and MAYV TRVL 4675 in Ae. aegypti based on the detection of plus-strand and minus-strand viral RNAs showing that both viruses disseminated from the mosquito midgut at similar timepoints (270).

In this study, we compare the vector competence of two Ae. aegypti strains, HWE and ORL for the two genotype D MAYV strains TRVL 4675 (termed "TRVL") and IQT 4335 (termed "IQT"). Previously, we used the same mosquito strains to assess their vector competence for CHIKV (strain 37997) from Senegal under similar laboratory conditions, which enables us to compare the interactions of both mosquito strains with CHIKV and MAYV (14). We also tested the vector competence of a locally-caught Ae. albopictus population for MAYV, confirming that naive mosquito populations can be highly susceptible for exotic arboviruses. So far, only partial genome sequences have been available for both MAYV strains, TRVL and IQT. Here, we present the full length genomes of both virus strains compare their phylogenetic relationships with other full-length MAYV genomes. Current outbreaks of CHIKV in South- and Central America increase the likelihood that MAYV and CHIKV may be able to co-circulate in common 
regions. In line with such a scenario, incidents of co-infections in patients involving CHIKV and flaviviruses such as dengue (1-4) viruses (DENV1-4; Flaviviridae; Flavivirus) or ZIKV have been recently reported from the Americas and the Caribbean (271-275). Thus, we decided to investigate how mixed infections between MAYV and CHIKV, when acquired by mosquitoes as coinfections or superinfections, would affect their ability to co-transmit both viruses.

\section{Results}

\section{Phylogenetic characterization of MAYV strains IQT 4235 and TRVL 4675}

Sequencing of the complete genomes of both MAYV strains, IQT and TRVL, confirmed that both were of the D-genotype. Their nucleotide sequences differed by $2.8 \%$ (320 nucleotides) from each other. A phylogenetic analysis was conducted using the full-length nucleotide sequences of 26 geographically diverse MAYV strains (including TRVL and IQT). The analysis revealed that similar to TRVL 15537 from Trinidad, MAYV TRVL is ancestral to other genotype D MAYV strains including IQT, which clustered with two other strains from Peru (Fig. 23). When comparing those 26 MAYV strains, 10 amino acid residues within nsP1, nsP2, and nsP3 were specific for the two strains from Trinidad, TRVL 4675 and TRVL 15537, the latter isolated in 1957 from a Mansonia venezuelensis mosquito caught in the Rio Grande Forest (267) (Table 5). Unique for the TRVL4675 strain from 1954 were single amino acid substitutions in nsP1 and nsP2. The IQT strain had single unique amino acid substitutions in nsP1 and nsP3. Fewer amino acid substitutions were observed in the polypeptide encoding 


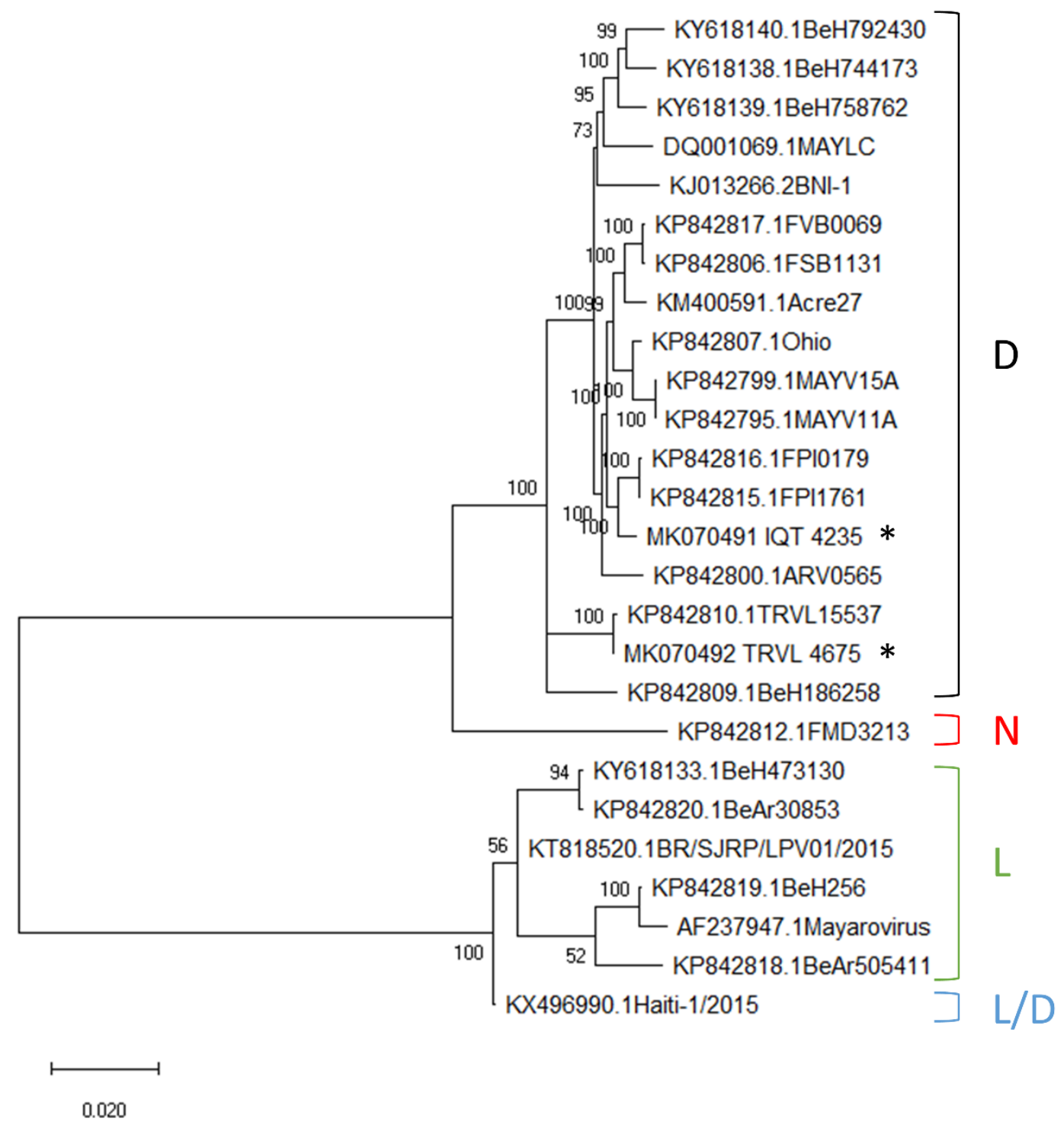

Figure 23. Phylogenetic analysis of full-length RNA genomes of geographically diverse MAYV strains/isolates.

The full-length nucleotide sequences of 26 MAYV strains including those of IQT $\left(^{*}\right)$ and TRVL $\left(^{*}\right)$ representing the genotypes Widely Dispersed $(=D)$, New $(=N)$, and Limited $(=L)$ were phylogenetically analyzed using the Maximum Likelihood algorithm based on the General Time Reversible model with bootstrap values of 1000 . For each virus strain, the GenBank accession number is indicated followed by its strain designation. The analysis was conducted in MEGA X. 
Table 5. Amino acid substitutions specific for MAYV strains TRLV 4675, TRVL 15537, and IQT 4235.

\begin{tabular}{|lccllcccc|}
\hline & nsP1 & nsP2 & nsP3 & nsP4 & C E3 & E2 & 6K/ & E1 \\
& & & & & & TF & \\
\hline TRVL 4675 I & T317I & V40A & F284L & & T59K & D50E \\
TRLV 15537 & T425I & K497R & D372V & & H130R & T136A \\
& S454N & T770S & R436S & & I175V & S343F \\
& & & E463D & & & T396I \\
TRVL 4675 & V386M & K41R & & & & T196I & \\
IQT 4235 & S445A & & T349I & & & \\
\hline
\end{tabular}


the structural proteins of the viruses as the E2 protein sequence had no unique residues found in the IQT strain, and only one unique substitution for the TRVL strain. However, both strains isolated from Trinidad shared seven specific amino acid substitutions in E2 and E1.

\section{Midgut and carcass infection of HWE and ORL mosquitoes with the IQT and TRVL strains of MAYV}

Comparison of the vector competence of Ae. aegypti strains HWE and ORL for MAYV strains IQT and TRVL clearly exposed differences in susceptibilities for the two virus strains. In both mosquito strains, median virus titers in midguts and carcasses were about 1 log pfu/ml higher for IQT than for TRVL during the seven day time course (Fig. 24A, B). In midgut tissues, median titers for both viruses did not change significantly over time although there was a tendency of increased median TRVL titers after 2 days pibm. However, both virus strains produced significantly increased median virus titers in carcasses at 7 days pibm indicating productive infection in secondary tissues. Interestingly, the median input titers of both viruses at time point zero $\left(\sim 10^{5} \mathrm{pfu} / \mathrm{ml}\right)$ were never exceeded in the midgut during the seven-day observation period and like-wise not in secondary tissues before 7 days pibm.

At 2 and 4 days pibm, MAYV IQT produced significantly higher mean Infection rates in midguts and carcasses of both mosquito strains in comparison to TRVL (Fig. 25A). Ninety-five percent of the ORL and all of the HWE samples contained detectable amounts of MAYV IQT as early as 2 days pibm. At the 

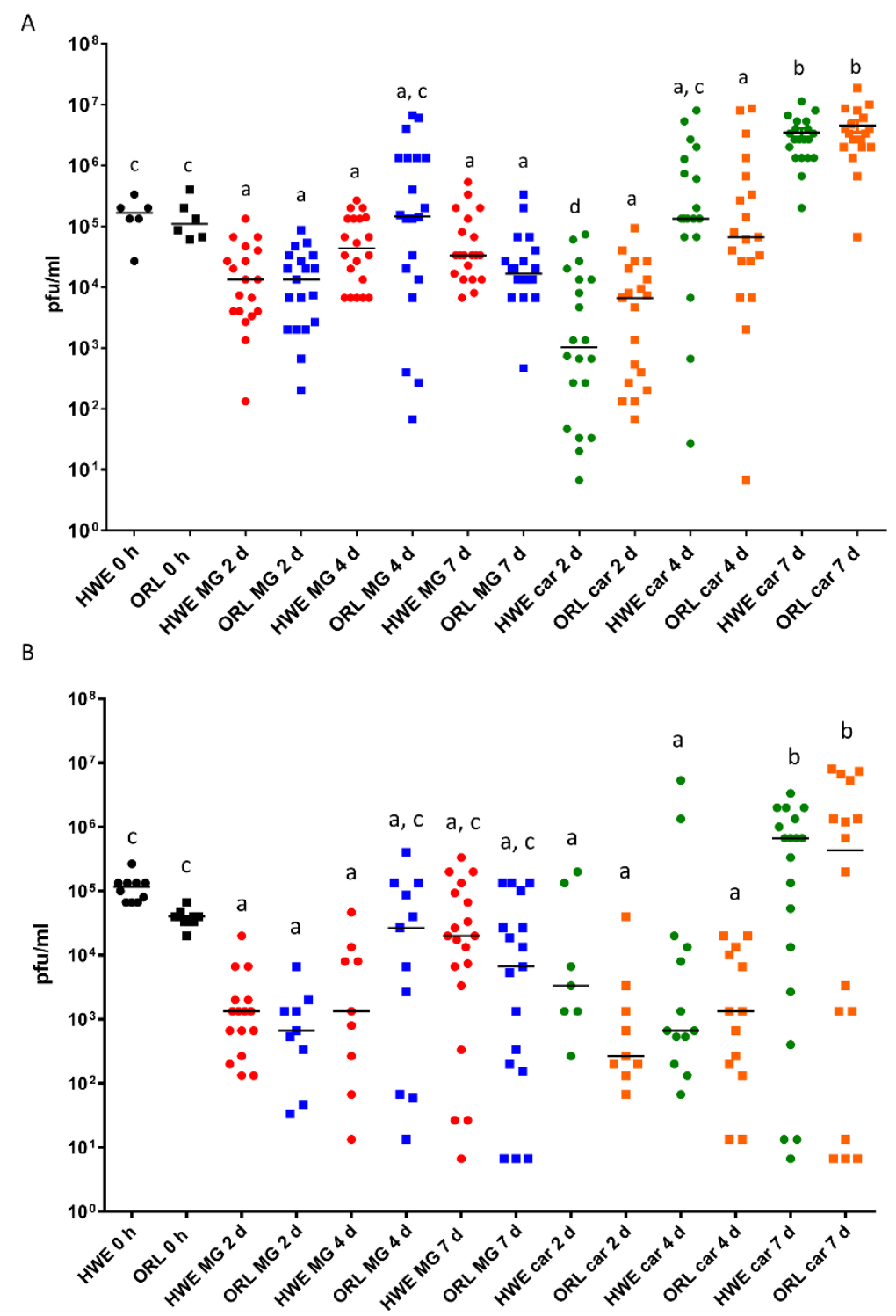

Figure 24. Intensities of MAYV IQT and TRVL infections in midguts and carcasses of Ae. aegypti strains HWE and ORL.

(A) MAYV IQT (artificial bloodmeal titer: $2.0 \times 10^{6}$ plaque forming units $(\mathrm{pfu}) / \mathrm{ml}$ ) and (B) MAYV TRVL (artificial bloodmeal titer: $\left.5.0 \times 10^{6} \mathrm{pfu} / \mathrm{ml}\right)$ titers in midguts $(n=20)$ and carcasses $(n=20)$ of individual HWE and ORL females were analyzed at $0,2,4$, and 7 days pibm by plaque assays in Vero cells. Each data point represents the MAYV titer of an individual midgut or carcass. For $0 \mathrm{~h}$, only whole-body females were assayed. The Mann-Whitney U-test was used for the statistical analysis to determine $P$ values. Only infected mosquitoes were included in the analysis. Black bars indicate medians. Different letters indicate median titers that were significantly different from each other. Significantly different comparisons: $P$ values ranged from $<0.0001$ to 0.0016 in (A) and from $<0.0001$ to 0.0004 in (B). 

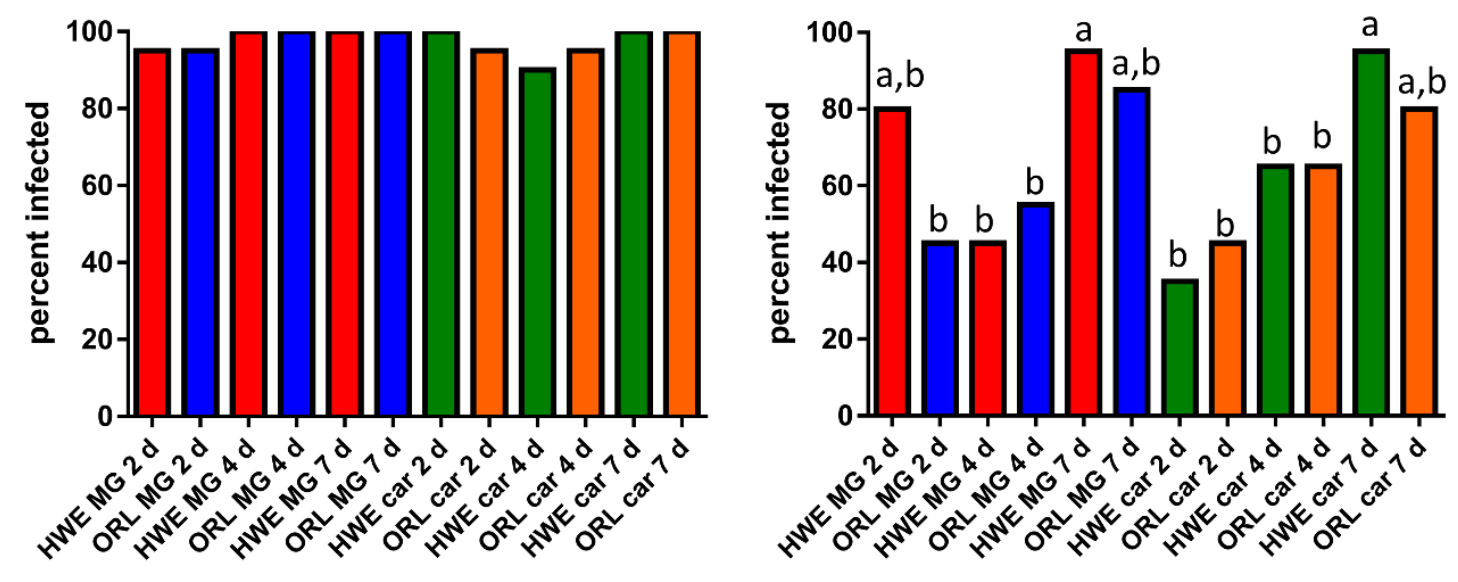

Figure 25. Prevalence of MAYV IQT and TRVL infections in midguts and carcasses of $A e$. aegypti strains HWE and ORL.

Rates of (A) MAYV IQT and (B) MAYV TRVL infections in midguts $(n=20)$ and carcasses $(n=20)$ of HWE and ORL mosquitoes at 2, 4, and 7 days pibm as analyzed by plaque assays in Vero cells. Fisher's exact test was used to determine $P$ values. Different letters indicate infection rates that were significantly different from each other. Significantly different comparisons in (B): $P$ values ranged from 0.0012 to 0.0484 . 
same time point, mean MAYV TRVL infection rates in midguts and carcasses were as low as $35 \%$ for HWE carcasses and $45 \%$ for HWE and ORL midguts (Fig. 25B). MAYV TRVL needed seven days to build up detectable titers in midguts and/or carcasses in $>80 \%$ of HWE and ORL mosquitoes. Thus, infection patterns varied more strongly between the two MAYV strains than between the two mosquito strains. Overall, MAYV TRVL was less infectious in HWE and ORL mosquitoes than MAYV IQT.

\section{MAYV head tissue infections and prevalence of the virus in saliva of HWE and ORL mosquitoes}

Arbovirus infection of head tissue is an indication that the virus has systemically infected the mosquito body including the salivary glands unless the virus is confronted with a strong salivary gland infection barrier. MAYV IQT infected head tissues of both mosquito strains ( $n=20$ for each virus and mosquito strain) with an infection rate of $>80 \%$ at 4 days pibm and reached maximal titers at 7 days pibm (Fig. 26A, C). The proportion of virus containing saliva samples ( $n=20$ for each virus and mosquito strain) increased about two-fold (ORL) to three-fold (HWE) between 4 and 7 days pibm (Fig. 26D). In contrast, MAYV TRVL exhibited weaker infectivity in both mosquito strains, as it was undetectable in both head tissue and saliva of HWE and ORL mosquitoes until 4 days pibm (Fig. 26B-D). Based on head tissue infection rates, virus titers, and proportion of virus containing saliva samples the maximal level of infection for MAYV TRVL was reached at 7 days pibm. Apparently, both MAYV strains 
A

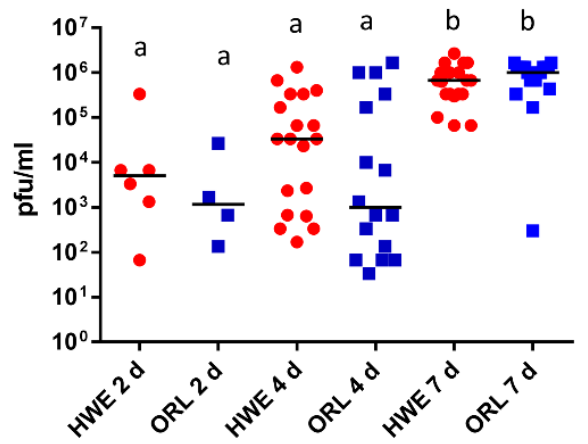

C

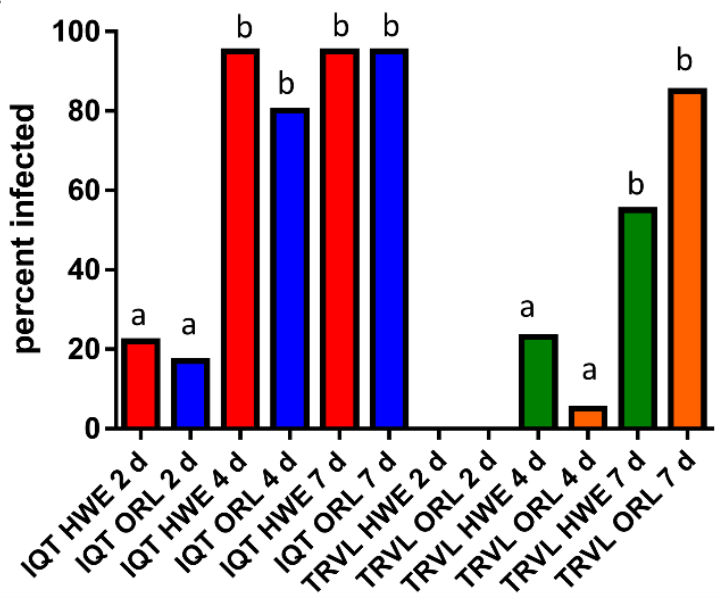

B

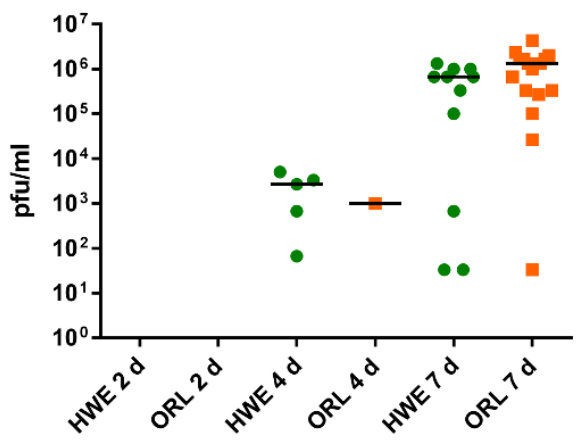

D

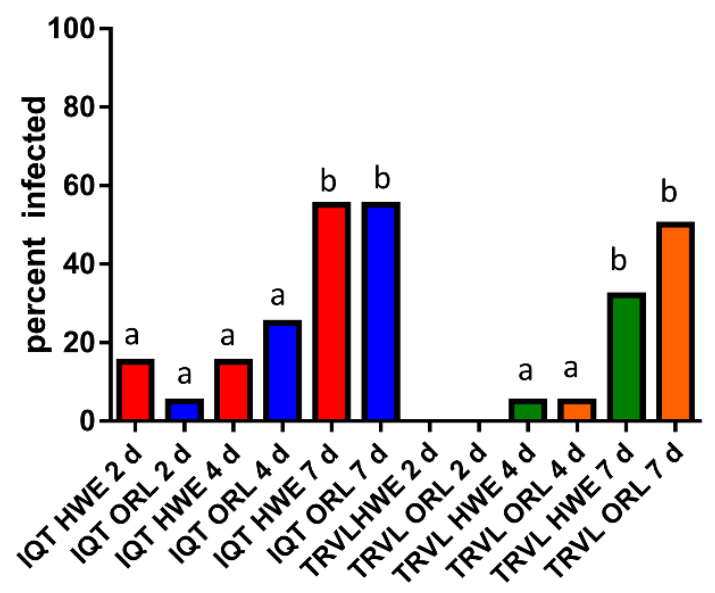

Figure 26. Intensities of infection and prevalence of MAYV IQT and TRVL in head tissues of Ae. aegypti strains HWE and ORL and prevalence of MAYV IQT and TRVL in HWE and ORL saliva samples.

(A) MAYV IQT and (B) TRVL titers in head tissues $(n=20)$ of individual HWE and ORL females were analyzed at 2, 4, and 7 days pibm by plaque assays in Vero cells. Each data point represents the MAYV titer of an individual head tissue. The Mann-Whitney U-test was used for the statistical analysis to determine $P$ values. Only infected mosquitoes were included in the analysis. Black bars indicate medians. Different letters indicate median titers that were significantly different from each other. Significantly different comparisons: $P$ values ranged from $<0.0001$ to 0.0045 . (C) Prevalence of MAYV IQT and TRVL infections in head tissues $(n=20)$ of HWE and ORL mosquitoes at 2, 4, and 7 days pibm. (C) and (D): different letters indicate infection rates that were significantly different from each other as analyzed by Fisher's exact test. Significantly different comparisons: $P$ values were $0.0012,0.0187$, and 0.0484 . (D) Prevalence of MAYV IQT and TRVL in saliva samples $(n=20)$ collected from each mosquito strain at 2,4 , and 7 days pibm. Significantly different comparisons: $P$ values were 0.0033 and 0.0436 . 
experienced a barrier at the salivary gland level in both HWE and ORL mosquitoes as up to $90 \%$ of head tissues were infected whereas the proportion of virus containing saliva samples barely exceeded $50 \%$. Similar to a weak relation between midgut virus titers and dissemination efficiencies, there was no clear correlation between MAYV titers in head tissue and virus excretion into saliva. The minimal extrinsic incubation period (EIP) for MAYV IQT and TRVL were 2 and 4 days pibm, respectively, in both mosquito strains. We observed a $50 \%$ EIP at 7 days pibm except for MAYV TRVL infected HWE with only $32 \%$ of saliva samples containing detectable virus at this time point.

\section{MAYV TRVL infection pattern in local Ae. albopictus}

Our newly established Ae. albopictus colony consists of offspring from a single female, which had been captured at the private residence of one of the authors in Columbia, Missouri. Exposing the offspring of this females to artificial bloodmeals occasionally resulted in poor feeding rates $(<10 \%)$ whereas at other occasions (in subsequent generations), feeding rates exceeded $60 \%$. We also noticed that hatch rates were rather irregular between generations when following the same procedure for our Ae. aegypti colony. However, when applying a "pressure hatching" procedure (see: Methods section), hatch rates became more stable. A standard PCR analysis followed by Sanger sequencing of amplicons revealed that the mosquitoes carry the Wolbachia endosymbiont (data not shown). We orally challenged Ae. albopictus (CoMO) females with MAYV TRVL. Our results showed an infection pattern of MAYV TRVL in Ae. 
albopictus that closely resembled those observed in our two Ae. aegypti laboratory strains (Fig. 27). About $90 \%$ of all midguts were infected with the virus between 2 and 4 days pibm indicating that there was virtually no midgut infection barrier for the virus. Median titers were between $10^{3}$ and $10^{4} \mathrm{pfu} /$ midgut, which were similar to those of the virus in Ae. aegypti (Fig. 24B). Curiously, at 4 days pibm, the midgut virus titers were $\sim 1$ log lower than at 2 days pibm. Midgut dissemination rates were between $70-90 \%$ at 2 and 4 days pibm, which were higher than those observed for the same MAYV strain in Ae. aegypti. Median MAYV TRVL titers (up to $10^{3} \mathrm{pfu} / \mathrm{ml}$ ) in carcasses of Ae. albopictus resembled those observed in Ae. aegypti. Following intrathoracic injection of 700 pfu/mosquito MAYV TRVL, median head tissue titers reached $\sim 10^{4} \mathrm{pfu}$ at $72 \mathrm{~h}$ post-injection. At that time-point, one third of the injected mosquitoes released the virus along with their saliva, allowing the conclusion that Ae. albopictus mosquitoes from Missouri can act as potential vectors for the virus.

\section{The effects of mixed infections between CHIKV 37997 and MAYV IQT on the transmission potential of both viruses}

We conducted two experiments to reveal the effects of mixed infections between CHIKV 37997 and MAYV IQT on the transmission of both viruses by Ae. aegypti. Initially, we established that our Taqman qRT-PCR assay would allow reliable, accurate virus-specific detection of viral genome copy number equivalents in mixed infections (Fig. 28A, B). Importantly, median bloodmeal 
A

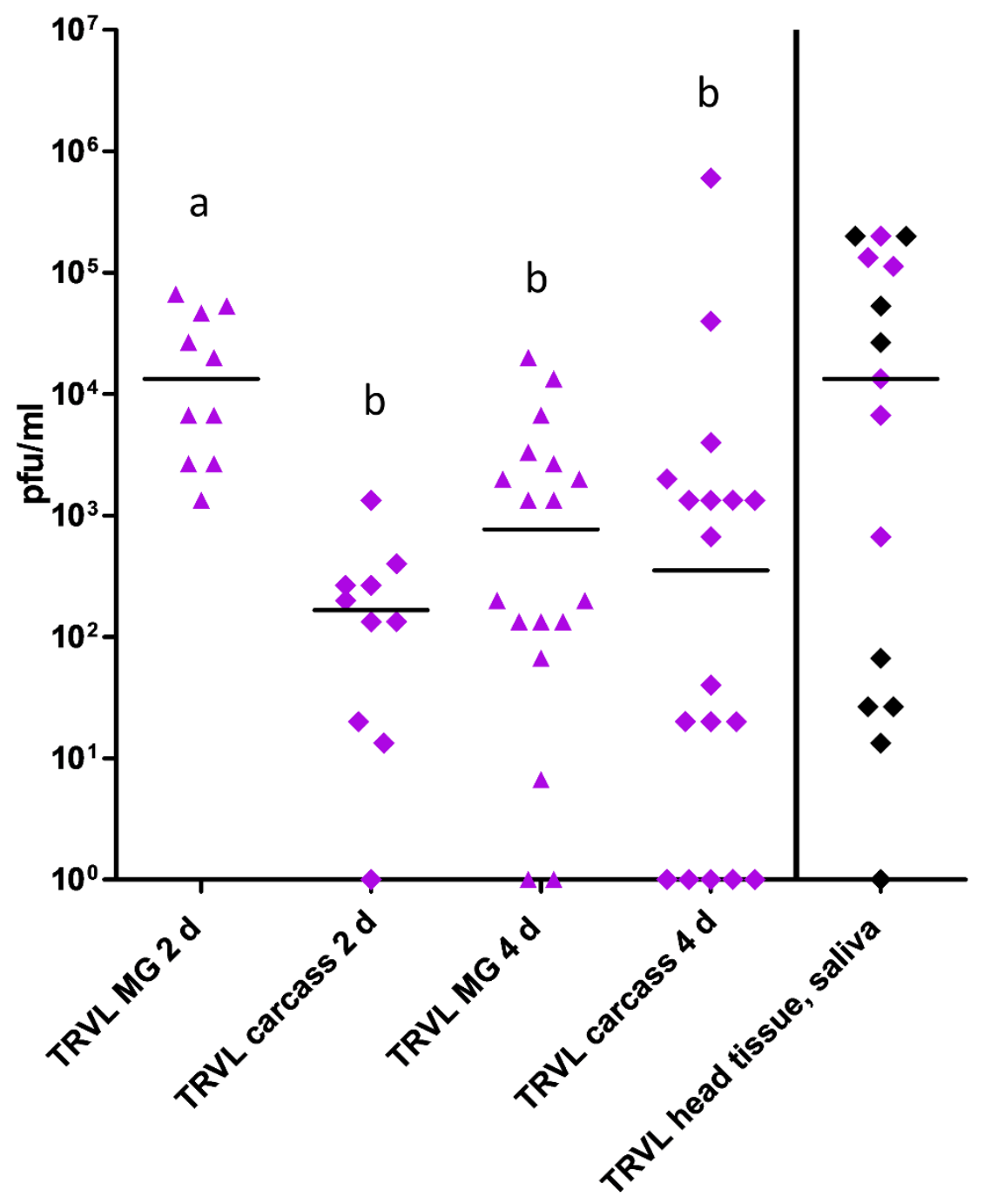

Figure 27. Intensities of MAYV TRVL infections in midguts, carcasses, and head tissues of $A e$. albopictus (CoMO), and prevalence of the virus in saliva samples.

(A) Virus titers in midguts and carcasses of $A e$. albopictus CoMO females were analyzed at 2 $(n=10)$ and 4 days $(n=18)$ pibm by plaque assays in Vero cells. Each data point represents the MAYV TRVL titer of an individual midgut or carcass. The virus titer in the artificial bloodmeal was $1 \times 10^{7} \mathrm{pfu} / \mathrm{ml}$. The Mann-Whitney U-test was used for the statistical analysis to determine $P$ values. Only infected mosquitoes were included in the analysis. Black bars indicate medians. Different letters indicate median titers that were significantly different from each other. Significantly different comparisons: $P$ values ranged from 0.0003 to 0.0013 . (B) Virus titers in head tissues were assessed at 3 days post-intrathoracic injection of 700 pfu/mosquito MAYV TRVL by plaque assays in Vero cells. Prevalence of the virus in saliva samples was assessed at 3 days post-intrathoracic injection by CPE analysis of Vero cells, which had been inoculated with the saliva samples. Black diamonds: MAYV TRVL titers in individual Ae. albopictus head tissues. Purple diamonds: head tissues samples that corresponded to virus containing saliva samples. 

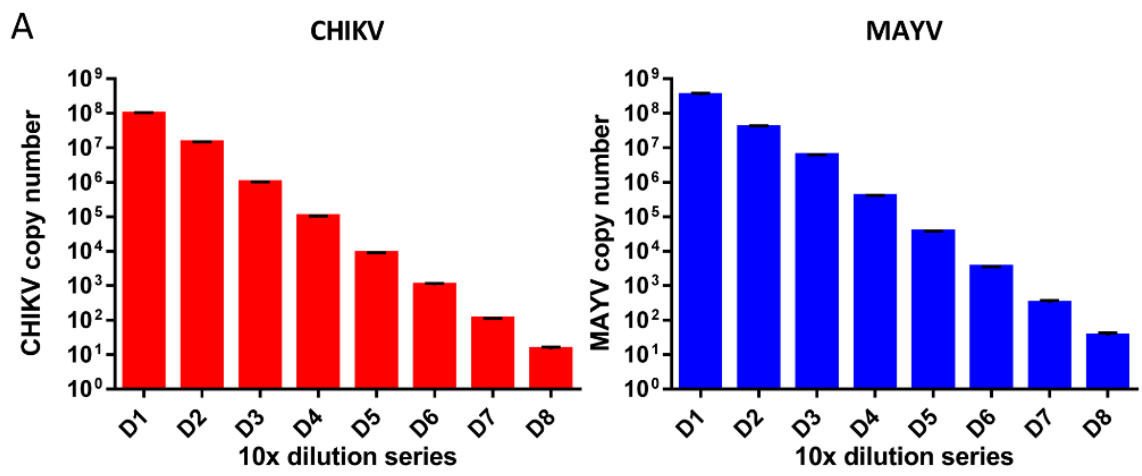

B

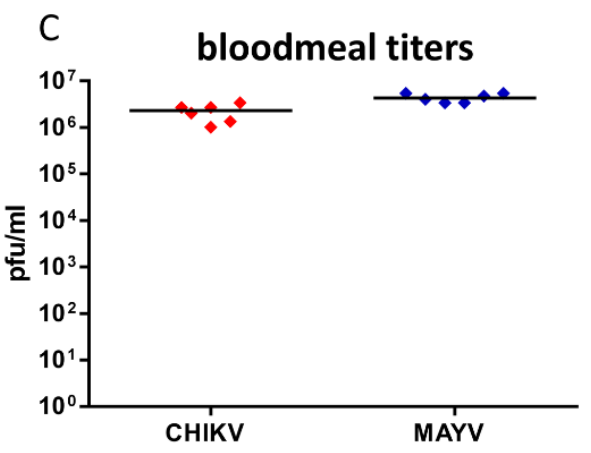

D

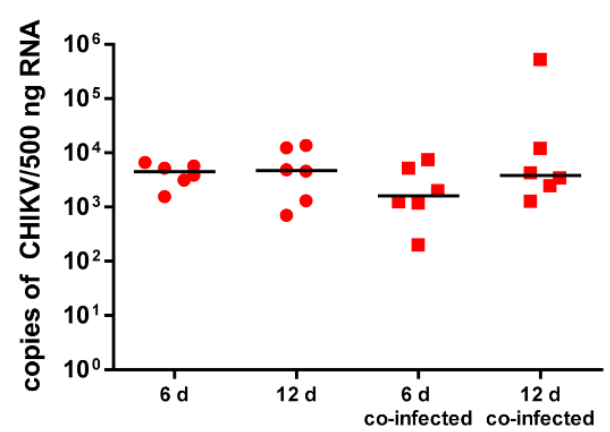

standard curves

\begin{tabular}{|l|l|l|}
\hline Detector & CHIKV & MAYV \\
\hline Slope & -3.36365 & -3.35221 \\
\hline Y-intercept & 39.32533 & 39.87649 \\
\hline $\mathrm{R}^{2}$ & 0.996763 & 0.997157 \\
\hline efficiency & $98.29 \%$ & $98.75 \%$ \\
\hline
\end{tabular}

E

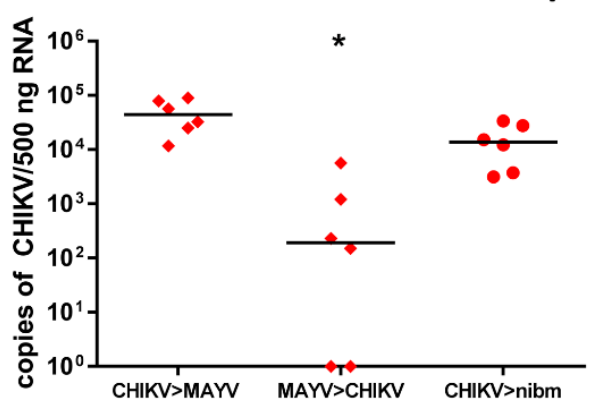

superinfections

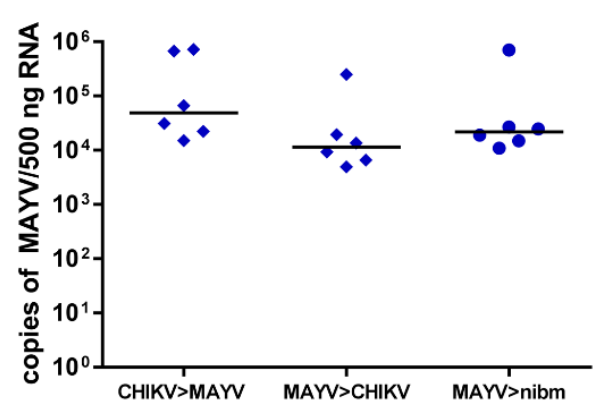


Figure 28. Effects of mixed infections between MAYV IQT and CHIKV 37997 on virus prevalence in mosquito saliva.

\begin{abstract}
(A) 10-fold dilution series values calculated from standard curves for CHIKV 37997 (red) and MAYV IQT (blue) showing specific detection of viral genome copy number equivalents. The templates for the standard curves consisted of a mixture of cDNAs (cloned into TA TOPO vector pCR 2.1 [Invitrogen]) derived from the genome sequences specific for CHIKV and MAYV. The reactions contained primers and Taqman probes for both viruses demonstrating that detection of each virus is specific and non-interfering. Error bars represent SD. (B) Standard curve quality parameters indicating high probability of virus specific detection of viral genome copy number equivalents. (C) Input (bloodmeal) titers (pfu/ml) of CHIKV and MAYV for the experiments in (D) and (E) as assessed via plaque assays in Vero cells. Bars indicate median values. (D) Coinfection of Ae. aegypti HWE with CHIKV 37997 and MAYV IQT. Left panel in red: CHIKV specific Taqman qRT-PCR detection of CHIKV genome copy number equivalents $/ 500 \mathrm{ng}$ total RNA in six saliva samples each representing a pool of saliva from five females $(n=30)$. Saliva samples were collected at 6 or 12 days pibm. Artificial bloodmeals contained CHIKV only or a mixture of CHIKV and MAYV. Right panel in blue: MAYV specific detection of MAYV genome copy number equivalents $/ 500 \mathrm{ng}$ total RNA in six saliva samples each representing a pool of saliva from five females $(n=30)$. Saliva samples were collected at 6 or 12 days pibm. Artificial bloodmeals contained MAYV only or the same mixture of CHIKV and MAYV as shown in the left chart (in red). Bars indicate median values. (E) Superinfections between CHIKV and MAYV. Left panel in red: CHIKV specific detection. Females acquired CHIKV via an artificial bloodmeal followed by a second bloodmeal (at 6 days post initial infectious bloodmeal) containing MAYV (CHIKV > MAYV). In another setup, females acquired MAYV via an artificial bloodmeal followed by a second bloodmeal (at 6 days post initial infectious bloodmeal) containing CHIKV (MAYV > $\mathrm{CHIKV)}$. As a control, females acquired CHIKV via artificial bloodfeeding and at 6 days post initial, infectious bloodmeal, females were given another, non-infectious bloodmeal (nibm). At 12 days post initial infectious bloodmeal, six saliva samples each consisting of a pool of saliva from five females were collected and analyzed $(n=30)$. Right panel in blue: MAYV specific detection of the same superinfection samples as shown in the left panel (in red). As a control, females acquired MAYV via artificial bloodfeeding and at 6 days post initial, infectious bloodmeal, females were given another, non-infectious bloodmeal (nibm). Samples were analyzed by Taqman qRTPCR from a standard curve as described above (D). The Kuskal-Wallis test was used for statistical analysis to determine $P$ values; ${ }^{*}$ indicates $p<0.020$. Bars indicate median values.
\end{abstract}


(input) titers were similar for both viruses (Fig. 28C), allowing a direct comparison between the two viruses in the mixed infection experiments. In the first experimental setup, mosquitoes simultaneously acquired MAYV and CHIKV via an artificial bloodmeal (co-infection). We chose the IQT strain of MAYV to compete with CHIKV 37997 because both viruses produced similar infection patterns and replication efficiencies in HWE mosquitoes (14). In a second experimental setup both viruses were subsequently acquired by the mosquitoes via artificial bloodmeals (superinfection). Each mosquito received two bloodmeals in a six-day interval. Either both bloodmeals contained different viruses or the first bloodmeal contained a virus whereas the second consisted of a noninfectious bloodmeal (nibm). In the co-infection experiment, both viruses reached similar concentrations in mosquito saliva at 12 days pibm, which amounted to $5 \mathrm{x}$ $10^{3}-1 \times 10^{4}$ median viral RNA copy number equivalents per pooled saliva sample (Fig. 28D). When CHIKV had been orally acquired before MAYV (MAYV superinfection), median viral RNA copy number equivalents in saliva were similar for both viruses and resembled the values obtained for mosquitoes that had acquired only one of the two viruses (Fig. 28E). Furthermore, when MAYV had been orally acquired before CHIKV (CHIKV superinfection), the median MAYV RNA copy number equivalent in saliva was similar to those obtained from mosquitoes that had acquired one of the two viruses only. However, the median CHIKV RNA copy number equivalent was significantly lower $(p=0.020$; and in two out of six pooled saliva samples [ $=10 / 30$ samples total] the virus was undetectable) of MAYV infected mosquitoes at 12 days pibm, suggesting that a 
primary MAYV infection led to a superinfection exclusion for CHIKV. In contrast, a primary CHIKV infection did not cause a superinfection exclusion for MAYV. In single infections (in absence of a second, non-infectious bloodmeal), CHIKV and MAYV produced similar median RNA copy number equivalents at 6 days pibm in saliva (Fig. 28D), suggesting that the low median CHIKV RNA copy number equivalent detected for CHIKV in saliva of MAYV infected mosquitoes was not based on principal differences in the two viruses' replication efficiencies. Instead, it is possible that prior infection with MAYV caused diminishment of cellular resources required for optimal CHIKV replication and infection in the mosquito. We also observed that at 6 days post-nibm, mosquitoes had increased (by $\sim 1$ log) median viral RNA genome copy equivalents in saliva in comparison to mosquitoes that had received only a single, virus-containing bloodmeal (Fig. 28D, E). This suggests that ingestion of additional, virus-free bloodmeals by the mosquito can have an enhancing effect for the acquired virus.

\section{Discussion}

Although MAYV has been known to circulate in South- and Central America for over 60 years, only a few vector-competence studies have been conducted to date $(32,268)$ including a very recent one by Wiggins and colleagues (269). Our results here show that the MAYV strains IQT and TRVL produced different infection patterns in two Ae. aegypti strains, HWE and ORL. Despite similar input titers, TRVL produced $\sim 1$ log lower median titers than IQT in midguts and carcasses of both Ae. aegypti strains throughout the time course 
study. Furthermore, MAYV IQT produced mean midgut and carcass infection rates of $>90 \%$ already at 2 days pibm, whereas the TRVL strain needed 7 days to generate similar mean infection rates. In field-obtained Ae. aegypti from Maynas Province of Peru, the earliest dissemination of MAYV IQT from the midgut to legs and wings was observed in $1 / 8$ mosquitoes at 3 days pibm and 29/31 mosquitoes were infected at 7 days pibm when the artificial bloodmeal titer of the virus was $\sim 10^{7} \mathrm{pfu} / \mathrm{ml}$ (32). Median MAYV TRVL titers and mean infection rates in midgut and carcasses of Ae. albopictus CoMO were similar to those observed in both Ae. aegypti strains, HWE and ORL. Furthermore, $50 \%$ of the tested Ae. albopictus individuals contained the virus in saliva at $72 \mathrm{~h}$ postintrathoracic injection. Due to relatively poor feeding rates of the mosquitoes on artificial bloodmeals containing virus-infected cell culture supernatants we did not test the presence of bloodmeal-acquired virus in saliva of Ae. albopictus. We were still able to show that MAYV disseminated from the midgut after an infectious bloodmeal had been orally acquired. Additionally, there were no extraordinary barriers to salivary gland infection and release of the intrathoracically injected virus from the salivary glands of Ae. albopictus CoMO. Infection rates of Brazilian Ae. albopictus, which had acquired MAYV TRVL 4675 via feeding on membrane feeder suspensions containing $10^{5}-10^{7} \mathrm{pfu} / \mathrm{ml}$ virus ranged between 13 and 85\%, respectively, at 15 days pibm (268). In Ae. albopictus CoMO at 2 days pibm, the same virus strain produced midgut and carcass infection rates of $89-100 \%$. Similar midgut infection rates were recently reported when evaluating MAYV TRVL infection patterns in Ae. albopictus from 
Florida (269). However, carcass infection rates in these mosquitoes did not exceed $50 \%$ at 3 and 6 days pibm, compared to $72-90 \%$ in Ae. albopictus CoMO at 2 and 4 days pibm. This suggests that the CoMO mosquitoes were more susceptible to MAYV-TRVL than the Ae. albopictus populations from Brazil and Florida. In wild-type Ae. aegypti from Florida, midgut infection and dissemination rates as well as midgut titers produced by the TRVL strain were similar to those observed in the laboratory-adapted HWE and ORL mosquitoes (269). However, $32 \%$ of the HWE saliva samples and $50 \%$ of the ORL saliva samples contained the virus at 7 days pibm in comparison to $<10 \%$ of the saliva samples obtained from the wild-type Ae. aegypti, indicating that the wild-types had a stronger barrier to the virus at the salivary gland level than the HWE or ORL strains.

Previously, we conducted a similar vector competence analysis in HWE and ORL mosquitoes for CHIKV (strain 37997) allowing us to directly compare the vector interactions of MAYV and CHIKV in these mosquitoes (14). Until 5 days pibm, median CHIKV titers were significantly ( $1 \mathrm{log})$ lower in midguts and carcasses of ORL mosquitoes than in those of the HWE strain. Overall, MAYV IQT produced an infection pattern similar to that of CHIKV 37997 in HWE midgut and carcasses, whereas the MAYV TRVL infection pattern in midgut and carcasses of both mosquito strains resembled that of CHIKV in ORL mosquitoes. In both Ae. aegypti strains, head tissue infections with MAYV TRVL were delayed by 2 days when compared to MAYV IQT correlating with a two-day delayed presence of the former in mosquito saliva. However, similar to CHIKV in our previous study, MAYV IQT was already detectable in head tissue and saliva at 2 
days pibm and regarding the presence of infectious virus in saliva, the barrier of infection at the salivary gland level for MAYV was similar to that for CHIKV as well $(14,32,276)$. EIPs as short as 2 days have been also reported for Venezuelan equine encephalitis virus (Togaviridae; Alphavirus; VEEV) in Ae. aegypti and Rift Valley fever virus (Bunyaviridae; Phlebovirus; RVFV) in Culex pipiens $(277,278)$.

Our data suggest that the IQT strain was better adapted to Ae. aegypti than MAYV TRVL. This finding is supported by the fact that earlier the IQT strain had been repeatedly passaged in Ae. albopictus C6/36 cells (267), whereas the TRVL strain had not been passaged in invertebrate cells. Thus, passage in C6/36 cells may have introduced adaptive mutations to the IQT strain resulting in a higher infectivity in Ae. aegypti in comparison to the TRVL strain, which had been maintained in mammalian cell lines $(73,259)$. Accordingly, it can be further speculated that the original mosquito vector for the TRVL strain was a species other than Ae. aegypti or Ae. albopictus. Full-length sequencing and phylogenetic analysis of the TRVL and IQT strains confirmed that both strains belong to the D genotype with MAYV TRVL clustering together with another strain from Trinidad, TRVL 15537. These two strains are more ancestral to MAYV IQT (and other D genotype viruses). Both Trinidad strains have specific amino acid residues in five viral proteins, nsP1, nsP2, nsP3, E2, and E1, whereas the IQT strain had specific amino acid residues only in nsP1 and nsP2. Further studies will be needed to elucidate which mutations would be required to adapt a forest dwelling mosquito 
transmitted MAYV strain to efficient transmission by urban, anthropophilic mosquito species, such as Ae. aegypti and Ae. albopictus.

We showed that simultaneously acquired MAYV and CHIKV (co-infection) can both be co-transmitted by the same vector with similar efficiencies, at least under laboratory conditions. These findings are in accordance with other studies demonstrating that in mosquitoes, co-infecting arboviruses do not interfere with each other $(76,279,280)$. In nature, co-infection of mosquitoes with several arboviruses is not an unlikely event, because human individuals in endemic regions have been frequently identified as being highly viremic for more than one mosquito-borne virus including DENV, CHIKV, and/or ZIKV $(271,273-275,281)$. Even more likely than co-infection is sequential arbovirus infection (superinfection) of mosquitoes in regions where different arboviruses cocirculate. Under this scenario the same mosquito could sequentially acquire different viruses when biting several hosts who would be viremic for different viruses. However, we observed superinfection exclusion for CHIKV when it was orally acquired by MAYV infected mosquitoes. In saliva of those mosquitoes, CHIKV but not MAYV, was absent or significantly less detectable. Interestingly, there was no interference for either virus when MAYV had been acquired by CHIKV infected mosquitoes. Superinfection exclusion, causing a cell persistently/productively infected with one virus to prevent infection with a second, closely related virus has been reported before for a range of arboviruses including alphaviruses $(282,283)$.Most of the studies addressing viral superinfection exclusion have been conducted in cell culture. For example, 
recently it was shown that Yellow fever virus (Flaviviridae; Flavivirus) and DENV exclude each other following sequential inoculation of C6/36 Ae. albopictus cells (284). Fewer studies have been conducted to analyze superinfection exclusion of arboviruses in vivo (285-290). Investigating superinfection exclusion gained additional attention when characterizing insect-specific flaviviruses such as Nhumirim virus, only found in Culex sp, or the insect-specific alphavirus Eilat virus. It was suggested that these mosquito-specific viruses may interfere with the replication and transmission of human-infecting arboviruses in the mosquito vector (291-293). Results of these studies were mixed; however, the presence of Nhumirim virus antagonized West Nile virus (Flaviviridae; Flavivirus) transmission by $C x$. quinquefasciatus, and the presence of Eilat virus delayed CHIKV dissemination from the midgut in Ae. aegypti by three days $(289,293)$. Our study demonstrates for the first time in vivo that two human-pathogenic alphaviruses can antagonize each other depending on their sequence of infection in the mosquito. Mechanistically, viral superinfection exclusion is not well understood. Reduced virion binding to cell surface receptors, cell entry capabilities, inhibition of nucleocapsid uncoating, and/or inhibition of viral replication complexes all have been described to explain the phenomenon for alphaviruses $(289,294)$. It will require extensive molecular analyses to reveal the mechanism underlying CHIKV exclusion in MAYV infected Ae. aegypti.

In summary, we confirmed in detailed studies that Ae. aegypti and Ae. albopictus can act as highly efficient vectors for MAYV as they have shown 
before for CHIKV and that under certain circumstances, both, CHIKV and MAYV, can be efficiently co-transmitted by the same infected mosquito. 


\section{CHAPTER VI}

\section{DISCUSSION}

\section{Potential roles of MMPs and their inhibitor, AeTIMP, in BL modification}

Activation of MMPs in mosquitoes which had ingested a bloodmeal suggests that these extra-cellular proteases have a role in $\mathrm{BL}$ degradation and/or in $\mathrm{BL}$ remodeling after a bloodmeal. Here we showed that AeMMP1 was inhibited by the general MMP inhibitor GM6001 and also by human Tissue Inhibitor of MMP 3 (HuTIMP3). AeMMP1 has also been shown to be inhibited by the recombinant TIMP from Ae. aegypti (AeTIMP) (150) in vitro. This study by Dong and colleagues also included transgenic overexpression of AeTIMP under the midgut specific, bloodmeal inducible carboxypeptidase A promoter (295). CHIKV infection in the presence of overexpressed AeTIMP led to higher dissemination rates from the midgut and increased whole body titers of orally acquired DENV4 (150). These results suggest that AeMMP inhibition enhances arbovirus dissemination from the midgut allowing the conclusion that these proteases might be important for $\mathrm{BL}$ remodeling after bloodmeal ingestion and digestion. These data are also in agreement with the higher expression levels of AeMMPs observed after $48 \mathrm{~h}$ pibm. However, the increased dissemination could also be due to direct interactions between AeTIMP and BL surface molecules. In human tissue culture for example, direct interaction of HuTIMP with ECM components independent of HuTIMP- HuMMP interactions has been well documented (296298). Obviously, in mosquitoes, the $B L$ degradation/remodeling mechanism 
needs to be further studied at the molecular level to determine the specific interactions between AeMMPs, AeTIMP, and their effects on the $\mathrm{BL}$ in vivo.

\section{Potential roles of other metalloproteinases in $B L$ remodeling}

In our studies, we focused on the characterization of AeMMPs. However, another prominent group of zinc-dependent, extracellular metalloproteinases, which have been extensively characterized in humans and so far only minimally described in arthropods, are ADAMs (A Disintegrin and Metalloproteinase), and ADAMTS (ADAM with thrombospondin motifs). ADAMs are transmembrane proteins which are structurally similar to MT-MMPs, except that they lack the hemopexin doman (299). These proteins have known functions in many biological processes during development and differentiation, such as acting as sheddases, collagenases, and having roles in tissue remodeling and metamorphosis (300-303). ADAMTS proteins are secreted but also retain similar functions such as cleavage of procollagens, versican, and other proteins present in the extracellular matrix (304-306). The genome of Drosophila encodes six ADAMTS genes (307) along with five ADAM genes (300), while the Ae. aegypti genome encodes six ADAMTS and seven ADAM genes. According to VectorBase genome searches and similar to MMPs, most of the Ae. aegypti ADAMs/ADAMTS possess the canonical zinc metalloproteinase catalytic domain motif HExxHxxGxxHD. So far, these proteins have not been characterized in $A e$ aegypti. Thus it would be interesting to investigate their activities during 
bloodmeal ingestion/digestion and arbovirus infections as they could also play an important role in $B L$ remodeling.

\section{MMP function in secondary tissues}

Here we localized AeMMPs to the midgut tissue; however, Drosophila MMP1 has also been shown to be present in salivary glands (135). The salivary glands of $A e$. aegypti are also surrounded by a BL that pathogens must pass through in order to productively infect these tissues $(308,309)$. Drosophila MMP1 has been shown to be upregulated in salivary glands during programmed cell death. This is accompanied by a decrease in Drosophila TIMP expression in these same tissues (310). Previously, we showed that CHIKV infection of salivary glands induces apoptosis (14). The present study has identified significantly increased AeMMP expression levels at timepoints concurring with $\mathrm{CHIKV}$ infection of carcass tissue that includes the salivary glands. However, so far we did not analyze MMP expression and activity in the salivary glands. It seems plausible to suggest that AeMMP activity may be important during alphavirus infection of the salivary glands in Ae. aegypti and further studies should be conducted to investigate this possibility.

\section{The tracheo-muscular complex in not involved in CHIKV dissemination}

Studies have shown that baculoviruses infect tracheal cells as a conduit to disseminate from the midgut in Lepidoptera $(145,190)$. Others have independently investigated whether in mosquitoes, the tracheal cell route is 
important for the dissemination of arboviruses from the midgut $(149,151,152,311)$. These experiments led to several hypotheses regarding the importance of the tracheal route in arbovirus dissemination from the midgut: 1) arboviruses infect the cytoplasm of a tracheal cell thereby bypassing the unmodified BL; 2) viruses escape from the midgut via the matrix of the modified $\mathrm{BL}$ and infect the tracheo-muscular complex; 3) arboviruses bud directly into the hemolymph from a previously infected muscle cell. Earlier TEM studies showed that intrathoracically injected alphaviruses infect midgut associated tracheae before associating with midgut tissue indicating that the tracheal cells might be involved in traversing the $\mathrm{BL}$ (148). Recently, we showed that CHIKV, when intrathoracically injected into bloodfed mosquitoes, was able to infect midgut tissue by bypassing the midgut associated tracheal cells. Our studies here indicate that CHIKV does not infect the midgut associated tracheae before disseminating from the midgut by traversing the modified $\mathrm{BL}$ adjacent to muscle tissue. Although we did observe CHIKV infection of tracheal cells at $40 \mathrm{~h}$ pibm, we suggest that $\mathrm{CHIKV}$ dissemination from the midgut does not depend on midgut associated-tracheal cell infection.

\section{Benefits of FIB-SEM imaging and 3D modeling}

The use of FIB-SEM for the first time in ultrastructural studies on arbovirus infected mosquito tissues convincingly identified $\mathrm{CHIK}$ virions localized between the inner and outer layers of the BL. This was clear evidence proving that $\mathrm{CHIKV}$ disseminates from the midgut of $A e$. aegypti by directly traversing the BL. We 
think that observing this phenomenon with high precision was only possible because we had the novel FIB-SEM technology on hand. Consequently, using FIB-SEM for other mosquito-arbovirus interaction studies involving ZIKV or DENV could lead to the discovery of their dissemination mechanisms from the mosquito midgut at the ultrastructural level. FIB-SEM could also be adapted to study other mosquito-pathogen systems such as infection of Anopheles with Plasmodium spp., and potentially aid in understanding how Wolbachia infection of Aedes species prevents the spread of arboviruses $(312,313)$.

\section{A universal dissemination mechanism for alphaviruses in Ae. aegypti}

MAYV is known to be naturally transmitted by Haemagogus spp., (30) but here we have confirmed that Ae. aegypti can be a competent vector for the virus under laboratory conditions. The IQT strain of MAYV had a minimal EIP of 2 days in Ae. aegypti, which is similar to that of CHIKV (14). In contrast, the TRVL strain of MAYV had a minimal EIP of 4 days. However, this strain was able to traverse the midgut between 32 and $48 \mathrm{~h}$ pibm when detected by taqman qRT-PCR, similar to the dissemination dynamics of CHIKV. MAYV and CHIKV are relatively closely related alphaviruses that belong to the Semliki Forest virus antigenic complex (314). These data allow the conclusion that there may be a universal escape mechanism from the midgut for these closely related alphaviruses in $A e$. aegypti, but further studies are needed to confirm this. However, preliminary observations so far suggest that other arboviruses such as flaviviruses like DENV or ZIKV may utilize a somewhat different strategy than alphaviruses to escape 
from the infected midgut to secondary tissues. This is attributed to the longer period of time required post-bloodmeal for detection of these viruses in secondary tissues (data not shown).

\section{Effects of alphaviral co-infection or super-infection of the mosquito vector on its ability to transmit both alphaviruses}

An interesting finding was the ability of $A e$. aegypti to effectively transmit both CHIKV and MAYV when simultaneously (co-)infected via artificial bloodmeals. Recently, similar observations have been made during another arbovirus co-infection experiment involving DENV2 and ZIKV (280). On the other hand, CHIKV and DENV2 co-infection of Ae. aegypti has led to increased DENV replication in comparison to that of CHIKV in the salivary glands (315). We observed a superinfection exclusion for CHIKV when systemic MAYV infection was established in the same mosquito before it was exposed to CHIKV via an infectious bloodmeal. We did not see the same effect on MAYV when systemic CHIKV infection was established. So far, only a few arboviral superinfection exclusion phenomena have been observed in vitro or in vivo $(284,289,290)$. More studies are needed to elucidate the mechanism of superinfection exclusion at the precise molecular level. Such investigations may require the use and development of novel tools, for example the use of single-cell-virology techniques in which single cells infected with multiple viruses could be isolated and examined for quantifiable viral RNA. 


\section{Potential benefits of generating a full-length infectious cDNA clone of MAYV}

A beneficial tool for the study of co-infection between CHIKV and MAYV in a mosquito vector would be the availability of full-length cDNA clones of both viruses modified to express two different fluorescent reporter genes. Full-length infectious cDNA clones have been previously generated for other alphaviruses including CHIKV, SINV, and Semliki Forest virus (316-318). This could be accomplished by inserting the MAYV genome sequence as a cDNA into a plasmid. To make the MAYV infectious cDNA clone beneficial for tracking dissemination from the midgut, a reporter gene such as EGFP could be inserted into the viral genome. This would eliminate the need for virus-specific antibodies to differentiate between several closely related alphaviruses in a mixed infection. One strategy to insert a reporter gene into the MAYV genome could be to place the reporter under the control of the duplicated viral subgenomic promoter. The resulting reporter expression cassette could be inserted into the cDNA either upstream or downstream of the viral structural genes (319). As shown before for other alphaviruses, both genome positions allow for reporter gene expression via an additional (recombinant) subgenomic RNA during viral replication in the cell $(232,234,319-323)$. An alternative position for the reporter gene would be its insertion between the non-structural genes nsP3 and nsP4. This method requires the reporter insert to be flanked by nsP2 protease-recognition sites for correct polyprotein processing and EGFP fluorescence detection (318). The availability of recombinant CHIKV and MAYV expressing two different reporters would allow 
co-infection studies to be conducted with the ability to track the two viruses specifically in various tissues of the vector. It would then be possible to detect in which tissue/s a preceding MAYV infection outcompetes CHIKV preventing the latter from being released in saliva. Another use of an infectious MAYV cDNA clone would be to introduce mutations to the viral genome to identify viral determinants potentially leading to superinfection exclusion in mixed infections.

\section{Conclusion}

This work represents substantial progress in our understanding of how alphaviruses infect, disseminate, and are transmitted by their principal vector, Ae. aegypti. It was important for us to investigate these aspects in in vivo studies using artificial, infectious bloodmeals as virus source that allow for easy standardization and reproducibility regarding the dose of infectious virus ingested by the mosquito. Taken together these studies have shown that early during bloodmeal digestion, alphaviruses are capable of infecting and replicating in the midgut epithelium of Ae. aegypti. Utilizing various sophisticated microscopy techniques and molecular approaches, we showed that CHIKV and MAYV disseminate from the mosquito midgut by directly traversing the $B L$ and begin replicating in secondary tissues between 32 and $48 \mathrm{~h}$ pibm. Furthermore, our data suggest that the $B L$ is a dynamic structure that is modified during stressful events such as ingestion and digestion of a bloodmeal and that the midgut of $\mathrm{Ae}$. aegypti releases proteases that are capable of interacting with the $B L$ leading to its temporal modification. These events are linked to both CHIKV and MAYV 
dissemination from the midgut, but it is still unclear if other arboviruses, flaviviruses such as DENV and ZIKV, are utilizing a similar midgut escape mechanism. Further studies should focus on the mechanism of midgut escape of other arboviruses not belonging to the alphaviruses. We have also investigated the potential for Ae. aegypti to transmit multiple alphaviruses simultaneously or sub-sequentially. As a result, a superinfection exclusion event was observed for CHIKV in systemically MAYV-infected mosquitoes but not vice versa. This suggests that two alphaviruses can have an antagonizing effect on each other in the same vector; however the molecular mechanism underlying this phenomenon remains elusive. 


\section{References}

1. Foster PG, de Oliveira TMP, Bergo ES, Conn JE, Sant'Ana DC, Nagaki SS, et al. Phylogeny of Anophelinae using mitochondrial protein coding genes. R Soc open Sci. 2017 Nov 8;4(11):170758.

2. Weeraratne TC, Surendran SN, Parakrama Karunaratne SHP. DNA barcoding of morphologically characterized mosquitoes belonging to the subfamily Culicinae from Sri Lanka. Parasit Vectors. 2018 Apr;11(1):266.

3. Dimopoulos G, Kafatos FC, Waters AP, Sinden RE. Malaria parasites and the anopheles mosquito. Chem Immunol. 2002;80:27-49.

4. Rezza G, Chen R, Weaver SC. O'nyong-nyong fever: a neglected mosquito-borne viral disease. Pathog Glob Health. 2017 Sep;111(6):2715 .

5. Mondet B, Vasconcelos PFC, Travassos da Rosa APA, Travassos da Rosa ES, Rodrigues SG, Travassos Rosa JFS, et al. Isolation of yellow fever virus from nulliparous Haemagogus (Haemagogus) janthinomys in eastern Amazonia. Vector Borne Zoonotic Dis. 2002;2(1):47-50.

6. Londono-Renteria B, Colpitts TM. A Brief Review of West Nile Virus Biology. Methods Mol Biol. 2016;1435:1-13.

7. Mansfield KL, Hernandez-Triana LM, Banyard AC, Fooks AR, Johnson N. Japanese encephalitis virus infection, diagnosis and control in domestic animals. Vet Microbiol. 2017 Mar;201:85-92.

8. Beltran FJ, Bechara YI, Guido GG, Cicuttin GL, Beaudoin JB, Gury Dohmen FE. Molecular detection of Saint Louis encephalitis virus in mosquitoes in Buenos Aires. Medicina (B Aires). 2014;74(6):433-6.

9. Borland EM, Ledermann JP, Powers AM. Culex Tarsalis Mosquitoes as Vectors of Highlands J Virus. Vector Borne Zoonotic Dis. 2016 Aug;16(8):558-65.

10. Ludlow M, Kortekaas J, Herden C, Hoffmann B, Tappe D, Trebst C, et al. Neurotropic virus infections as the cause of immediate and delayed neuropathology. Acta Neuropathol. 2015/12/10. 2016;131(2):159-84.

11. Kulasekera VL, Kramer L, Nasci RS, Mostashari F, Cherry B, Trock SC, et al. West Nile virus infection in mosquitoes, birds, horses, and humans, Staten Island, New York, 2000. Emerg Infect Dis. 2001;7(4):722-5. 
12. Mutebi J-P, Gianella A, Travassos da Rosa A, Tesh RB, Barrett ADT, Higgs S. Yellow fever virus infectivity for Bolivian Aedes aegypti mosquitoes. Emerg Infect Dis. 2004 Sep;10(9):1657-60.

13. Poole-Smith BK, Hemme RR, Delorey M, Felix G, Gonzalez AL, Amador $\mathrm{M}$, et al. Comparison of vector competence of Aedes mediovittatus and Aedes aegypti for dengue virus: implications for dengue control in the Caribbean. PLoS Negl Trop Dis. 2015 Feb 6;9(2):e0003462-e0003462.

14. Dong S, Kantor AM, Lin J, Passarelli AL, Clem RJ, Franz AWE. Infection pattern and transmission potential of chikungunya virus in two New World laboratory-adapted Aedes aegypti strains. Sci Rep. 2016 Apr;6:24729.

15. Le Coupanec A, Babin D, Fiette L, Jouvion G, Ave P, Misse D, et al. Aedes mosquito saliva modulates Rift Valley fever virus pathogenicity. PLoS Negl Trop Dis. 2013 Jun 13;7(6):e2237-e2237.

16. Epelboin Y, Talaga S, Epelboin L, Dusfour I. Zika virus: An updated review of competent or naturally infected mosquitoes. PLoS Negl Trop Dis. 2017 Nov 16;11(11):e0005933-e0005933.

17. Lim EXY, Lee WS, Madzokere ET, Herrero LJ. Mosquitoes as Suitable Vectors for Alphaviruses. Viruses. 2018 Feb 14;10(2):84.

18. Toivanen A. Alphaviruses: an emerging cause of arthritis? Curr Opin Rheumatol. 2008 Jul;20(4):486-90.

19. Santiago FW, Halsey ES, Siles C, Vilcarromero S, Guevara C, Silvas JA, et al. Long-Term Arthralgia after Mayaro Virus Infection Correlates with Sustained Pro-inflammatory Cytokine Response. PLoS Negl Trop Dis. 2015;9(10):e0004104.

20. Paixao ES, Rodrigues LC, Costa M da CN, Itaparica M, Barreto F, Gerardin P, et al. Chikungunya chronic disease: a systematic review and meta-analysis. Trans R Soc Trop Med Hyg. 2018 Jul;112(7):301-16.

21. Lasala PR, Holbrook M. Tick-borne flaviviruses. Clin Lab Med. 2010 Mar;30(1):221-35.

22. Powers AM, Roehrig JT. Alphaviruses. Methods Mol Biol. 2011;665:17-38.

23. Carver S, Bestall A, Jardine A, Ostfeld RS. Influence of hosts on the ecology of arboviral transmission: potential mechanisms influencing dengue, Murray Valley encephalitis, and Ross River virus in Australia. Vector Borne Zoonotic Dis. 2009 Feb;9(1):51-64. 
24. Akiner MM, Demirci B, Babuadze G, Robert V, Schaffner F. Spread of the Invasive Mosquitoes Aedes aegypti and Aedes albopictus in the Black Sea Region Increases Risk of Chikungunya, Dengue, and Zika Outbreaks in Europe. PLoS Negl Trop Dis. 2016 Apr 26;10(4):e0004664-e0004664.

25. Hawley WA, Reiter P, Copeland RS, Pumpuni CB, Craig GBJ. Aedes albopictus in North America: probable introduction in used tires from northern Asia. Science. 1987 May;236(4805):1114-6.

26. Vega-Rua A, Zouache K, Girod R, Failloux A-B, Lourenco-de-Oliveira R. High level of vector competence of Aedes aegypti and Aedes albopictus from ten American countries as a crucial factor in the spread of Chikungunya virus. J Virol. 2014 Jun;88(11):6294-306.

27. Gratz NG. Critical review of the vector status of Aedes albopictus. Med Vet Entomol. 2004 Sep;18(3):215-27.

28. Powell JR, Tabachnick WJ. History of domestication and spread of Aedes aegypti--a review. Mem Inst Oswaldo Cruz. 2013 Dec;108 Suppl(Suppl 1):11-7.

29. Kraemer MUG, Sinka ME, Duda KA, Mylne AQN, Shearer FM, Barker CM, et al. The global distribution of the arbovirus vectors Aedes aegypti and Ae. albopictus. Elife. 2015;4:e08347.

30. Hoch AL, Peterson NE, LeDuc JW, Pinheiro FP. An outbreak of Mayaro virus disease in Belterra, Brazil. III. Entomological and ecological studies. Am J Trop Med Hyg. 1981 May;30(3):689-98.

31. Alencar J, Lorosa ES, Degallier IN, Serra-Freire NM, Pacheco JB, Guimaraes AE. Feeding patterns of Haemagogus janthinomys (Diptera: Culicidae) in different regions of Brazil. J Med Entomol. 2005 Nov;42(6):981-5.

32. Long KC, Ziegler SA, Thangamani S, Hausser NL, Kochel TJ, Higgs S, et al. Experimental transmission of Mayaro virus by Aedes aegypti. Am J Trop Med Hyg. 2011 Oct;85(4):750-7.

33. Foster WA. Mosquito sugar feeding and reproductive energetics. Annu Rev Entomol. 1995;40:443-74.

34. Dhadialla TS, Raikhel AS. Biosynthesis of mosquito vitellogenin. J Biol Chem. 1990 Jun;265(17):9924-33.

35. Burkot TR, Handzel T, Schmaedick MA, Tufa J, Roberts JM, Graves PM. Productivity of natural and artificial containers for Aedes polynesiensis and 
Aedes aegypti in four American Samoan villages. Med Vet Entomol. 2007 Mar;21(1):22-9.

36. Mangudo C, Aparicio JP, Gleiser RM. Tree holes as larval habitats for Aedes aegypti in urban, suburban and forest habitats in a dengue affected area. Bull Entomol Res. 2015 Dec;105(6):679-84.

37. Harrington LC, Scott TW, Lerdthusnee K, Coleman RC, Costero A, Clark GG, et al. Dispersal of the dengue vector Aedes aegypti within and between rural communities. Am J Trop Med Hyg. 2005 Feb;72(2):209-20.

38. Scott TW, Naksathit A, Day JF, Kittayapong P, Edman JD. A fitness advantage for Aedes aegypti and the viruses it transmits when females feed only on human blood. Am J Trop Med Hyg. 1997 Aug;57(2):235-9.

39. Ponlawat A, Harrington LC. Blood feeding patterns of Aedes aegypti and Aedes albopictus in Thailand. J Med Entomol. 2005 Sep;42(5):844-9.

40. Beerntsen BT, James AA, Christensen BM. Genetics of mosquito vector competence. Microbiol Mol Biol Rev. 2000 Mar;64(1):115-37.

41. Bosio CF, Beaty BJ, Black WC 4th. Quantitative genetics of vector competence for dengue-2 virus in Aedes aegypti. Am J Trop Med Hyg. 1998 Dec;59(6):965-70.

42. McFarlane M, Arias-Goeta C, Martin E, O'Hara Z, Lulla A, Mousson L, et al. Characterization of Aedes aegypti innate-immune pathways that limit Chikungunya virus replication. PLoS Negl Trop Dis. 2014 Jul;8(7):e2994.

43. Anglero-Rodriguez YI, MacLeod HJ, Kang S, Carlson JS, Jupatanakul N, Dimopoulos G. Aedes aegypti Molecular Responses to Zika Virus: Modulation of Infection by the Toll and Jak/Stat Immune Pathways and Virus Host Factors. Front Microbiol. 2017;8:2050.

44. Jupatanakul N, Sim S, Angleró-Rodríguez YI, Souza-Neto J, Das S, Poti $\mathrm{KE}$, et al. Engineered Aedes aegypti JAK/STAT Pathway-Mediated Immunity to Dengue Virus. PLoS Negl Trop Dis. 2017 Jan 12;11(1):e0005187.

45. Khoo CCH, Piper J, Sanchez-Vargas I, Olson KE, Franz AWE. The RNA interference pathway affects midgut infection- and escape barriers for Sindbis virus in Aedes aegypti. BMC Microbiol. 2010 Apr;10:130.

46. Carvalho-Leandro D, Ayres CFJ, Guedes DRD, Suesdek L, Melo-Santos MA V, Oliveira CF, et al. Immune transcript variations among Aedes aegypti populations with distinct susceptibility to dengue virus serotype 2 . 
Acta Trop. 2012 Nov;124(2):113-9.

47. Heitmann A, Jansen S, Lühken R, Leggewie M, Badusche M, Pluskota B, et al. Experimental transmission of Zika virus by mosquitoes from central Europe. Euro Surveill Bull Eur sur les Mal Transm = Eur Commun Dis Bull. 2017 Jan;22(2).

48. Hamer GL. Heterogeneity of Mosquito (Diptera: Culicidae) Control Community Size, Research Productivity, and Arboviral Diseases Across the United States. J Med Entomol. 2016 May;53(3):485-95.

49. Hillyer JF, Schmidt SL, Fuchs JF, Boyle JP, Christensen BM. Ageassociated mortality in immune challenged mosquitoes (Aedes aegypti) correlates with a decrease in haemocyte numbers. Cell Microbiol. 2005 Jan;7(1):39-51.

50. Fellous S, Lazzaro BP. Larval food quality affects adult (but not larval) immune gene expression independent of effects on general condition. Mol Ecol. 2010 Apr;19(7):1462-8.

51. Kramer LD, Ciota AT. Dissecting vectorial capacity for mosquito-borne viruses. Curr Opin Virol. 2015 Dec;15:112-8.

52. Goindin D, Delannay C, Ramdini C, Gustave J, Fouque F. Parity and longevity of Aedes aegypti according to temperatures in controlled conditions and consequences on dengue transmission risks. PLoS One. 2015 Aug 10;10(8):e0135489-e0135489.

53. Tjaden NB, Thomas SM, Fischer D, Beierkuhnlein C. Extrinsic Incubation Period of Dengue: Knowledge, Backlog, and Applications of Temperature Dependence. PLoS Negl Trop Dis. 2013 Jun 27;7(6):e2207-e2207.

54. Carrington LB, Simmons CP. Human to mosquito transmission of dengue viruses. Front Immunol. 2014 Jun 17;5:290.

55. Scott TW, Clark GG, Lorenz LH, Amerasinghe PH, Reiter P, Edman JD. Detection of multiple blood feeding in Aedes aegypti (Diptera: Culicidae) during a single gonotrophic cycle using a histologic technique. J Med Entomol. 1993 Jan;30(1):94-9.

56. Getis A, Morrison AC, Gray K, Scott TW. Characteristics of the spatial pattern of the dengue vector, Aedes aegypti, in lquitos, Peru. Am J Trop Med Hyg. 2003 Nov;69(5):494-505.

57. Stoddard ST, Morrison AC, Vazquez-Prokopec GM, Paz Soldan V, Kochel $\mathrm{TJ}$, Kitron $\mathrm{U}$, et al. The role of human movement in the transmission of 
vector-borne pathogens. PLoS Negl Trop Dis. 2009 Jul;3(7):e481.

58. Chan M, Johansson MA. The incubation periods of Dengue viruses. PLoS One. 2012 Nov 30;7(11):e50972-e50972.

59. Liu Z, Zhang Z, Lai Z, Zhou T, Jia Z, Gu J, et al. Temperature Increase Enhances Aedes albopictus Competence to Transmit Dengue Virus. Vol. 8, Frontiers in Microbiology. 2017. p. 2337.

60. Scott TW, Takken W. Feeding strategies of anthropophilic mosquitoes result in increased risk of pathogen transmission. Trends Parasitol. 2012 Mar;28(3):114-21.

61. Figueiredo LTM. Emergent arboviruses in Brazil. Rev Soc Bras Med Trop. 2007;40(2):224-9.

62. Mavian C, Rife BD, Dollar JJ, Cella E, Ciccozzi M, Prosperi MCF, et al. Emergence of recombinant Mayaro virus strains from the Amazon basin. Sci Rep. 2017 Aug;7(1):8718.

63. Mucci LF, Medeiros-Sousa AR, Ceretti-Junior W, Fernandes A, Camargo AA, Evangelista E, et al. Haemagogus leucocelaenus and Other Mosquitoes Potentially Associated With Sylvatic Yellow Fever In Cantareira State Park In the Sao Paulo Metropolitan Area, Brazil. J Am Mosq Control Assoc. 2016 Dec;32(4):329-32.

64. Acosta-Ampudia Y, Monsalve DM, Rodríguez Y, Pacheco Y, Anaya J-M, Ramírez-Santana C. Mayaro: an emerging viral threat? Emerg Microbes Infect. 2018 Sep 26;7(1):163.

65. Reed KD, Meece JK, Henkel JS, Shukla SK. Birds, migration and emerging zoonoses: west nile virus, lyme disease, influenza $A$ and enteropathogens. Clin Med Res. 2003 Jan;1(1):5-12.

66. Weise WJ, Hermance ME, Forrester N, Adams AP, Langsjoen R, Gorchakov R, et al. A Novel Live-Attenuated Vaccine Candidate for Mayaro Fever. PLoS Negl Trop Dis. 2014 Aug 7;8(8):e2969.

67. Yap ML, Klose T, Urakami A, Hasan SS, Akahata W, Rossmann MG. Structural studies of Chikungunya virus maturation. Proc Natl Acad Sci U S A. 2017/12/04. 2017 Dec 26;114(52):13703-7.

68. Strauss JH, Strauss EG. The alphaviruses: gene expression, replication, and evolution. Microbiol Rev. 1994 Sep;58(3):491-562.

69. Rupp JC, Sokoloski KJ, Gebhart NN, Hardy RW. Alphavirus RNA synthesis 
and non-structural protein functions. J Gen Virol. 2015 Sep;96(9):2483500 .

70. Simizu B, Yamamoto K, Hashimoto K, Ogata T. Structural proteins of Chikungunya virus. J Virol. 1984 Jul;51(1):254-8.

71. Lumsden WH. An epidemic of virus disease in Southern Province, Tanganyika Territory, in 1952-53. II. General description and epidemiology. Trans R Soc Trop Med Hyg. 1955 Jan;49(1):33-57.

72. Pan American Health Organization. Report on Chikungunya Virus Transmission and Its Impact in the Region of the Americas. Washington (DC); 2015.

73. Anderson CR, Downs WG, Wattley GH, Ahin NW, Reese AA. Mayaro virus: a new human disease agent. II. Isolation from blood of patients in Trinidad, B.W.I. Am J Trop Med Hyg. 1957 Nov;6(6):1012-6.

74. Halsey ES, Siles C, Guevara C, Vilcarromero S, Jhonston EJ, Ramal C, et al. Mayaro virus infection, Amazon Basin region, Peru, 2010-2013. Emerg Infect Dis. 2013 Nov;19(11):1839-42.

75. Lednicky J, De Rochars VMB, Elbadry M, Loeb J, Telisma T, Chavannes S, et al. Mayaro Virus in Child with Acute Febrile Illness, Haiti, 2015. Emerg Infect Dis. 2016 Nov;22(11):2000-2.

76. Goertz GP, Vogels CBF, Geertsema C, Koenraadt CJM, Pijlman GP. Mosquito co-infection with Zika and chikungunya virus allows simultaneous transmission without affecting vector competence of Aedes aegypti. PLoS Negl Trop Dis. 2017 Jun;11(6):e0005654.

77. Paniz-Mondolfi AE, Rodriguez-Morales AJ, Blohm G, Marquez M, VillamilGomez WE. ChikDenMaZika Syndrome: the challenge of diagnosing arboviral infections in the midst of concurrent epidemics. Ann Clin Microbiol Antimicrob. 2016 Jul 22;15(1):42.

78. Cunha RV da, Trinta KS. Chikungunya virus: clinical aspects and treatment - A Review. Mem Inst Oswaldo Cruz. 2017 Aug;112(8):523-31.

79. McGill PE. Viral infections: alpha-viral arthropathy. Baillieres Clin Rheumatol. 1995 Feb;9(1):145-50.

80. Schwartz $\mathrm{O}$, Albert ML. Biology and pathogenesis of chikungunya virus. Nat Rev Microbiol. 2010 Jul;8(7):491-500.

81. Pinheiro FP, Freitas RB, Travassos da Rosa JF, Gabbay YB, Mello WA, 
LeDuc JW. An outbreak of Mayaro virus disease in Belterra, Brazil. I. Clinical and virological findings. Am J Trop Med Hyg. 1981 May;30(3):67481.

82. Theilacker C, Held J, Allering L, Emmerich P, Schmidt-Chanasit J, Kern W $V$, et al. Prolonged polyarthralgia in a German traveller with Mayaro virus infection without inflammatory correlates. BMC Infect Dis. 2013 Aug;13:369.

83. Hua C, Combe B. Chikungunya Virus-Associated Disease. Curr Rheumatol Rep. 2017 Oct;19(11):69.

84. Thiberville SD, Moyen N, Dupuis-Maguiraga L, Nougairede A, Gould EA, Roques $\mathrm{P}$, et al. Chikungunya fever: epidemiology, clinical syndrome, pathogenesis and therapy. Antiviral Res. 2013 Sep;99(3):345-70.

85. Masclee GMC, Valkhoff VE, Coloma PM, de Ridder M, Romio S, Schuemie $\mathrm{MJ}$, et al. Risk of upper gastrointestinal bleeding from different drug combinations. Gastroenterology. 2014 Oct;147(4):784.

86. Gallegos KM, Drusano GL, D Argenio DZ, Brown AN. Chikungunya Virus: In Vitro Response to Combination Therapy With Ribavirin and Interferon Alfa 2a. J Infect Dis. 2016/08/05. 2016 Oct 15;214(8):1192-7.

87. Tsetsarkin KA, McGee CE, Volk SM, Vanlandingham DL, Weaver SC, Higgs S. Epistatic roles of E2 glycoprotein mutations in adaption of chikungunya virus to Aedes albopictus and Ae. aegypti mosquitoes. PLoS One. 2009 Aug;4(8):e6835.

88. Kielian M, Rey FA. Virus membrane-fusion proteins: more than one way to make a hairpin. Nat Rev Microbiol. 2006 Jan;4(1):67-76.

89. van Duijl-Richter MKS, Hoornweg TE, Rodenhuis-Zybert IA, Smit JM. Early Events in Chikungunya Virus Infection-From Virus Cell Binding to Membrane Fusion. Viruses. 2015 Jul;7(7):3647-74.

90. Jose J, Snyder JE, Kuhn RJ. A structural and functional perspective of alphavirus replication and assembly. Future Microbiol. 2009 Sep;4(7):83756 .

91. Li G, Rice CM. The signal for translational readthrough of a UGA codon in Sindbis virus RNA involves a single cytidine residue immediately downstream of the termination codon. J Virol. 1993 Aug;67(8):5062-7.

92. Strauss EG, De Groot RJ, Levinson R, Strauss JH. Identification of the active site residues in the nsP2 proteinase of Sindbis virus. Virology. 1992 
Dec;191(2):932-40.

93. de Groot RJ, Hardy WR, Shirako Y, Strauss JH. Cleavage-site preferences of Sindbis virus polyproteins containing the non-structural proteinase.

Evidence for temporal regulation of polyprotein processing in vivo. EMBO J. 1990 Aug;9(8):2631-8.

94. Hahn YS, Grakoui A, Rice CM, Strauss EG, Strauss JH. Mapping of RNAtemperature-sensitive mutants of Sindbis virus: complementation group $\mathrm{F}$ mutants have lesions in nsP4. J Virol. 1989 Mar;63(3):1194-202.

95. Lemm JA, Rumenapf T, Strauss EG, Strauss JH, Rice CM. Polypeptide requirements for assembly of functional Sindbis virus replication complexes: a model for the temporal regulation of minus- and plus-strand RNA synthesis. EMBO J. 1994 Jun;13(12):2925-34.

96. Cross RK. Identification of a unique guanine-7-methyltransferase in Semliki Forest virus (SFV) infected cell extracts. Virology. 1983 Oct;130(2):452-63.

97. Mi S, Stollar V. Expression of Sindbis virus nsP1 and methyltransferase activity in Escherichia coli. Virology. 1991 Sep;184(1):423-7.

98. Weiss B, Geigenmuller-Gnirke U, Schlesinger S. Interactions between Sindbis virus RNAs and a 68 amino acid derivative of the viral capsid protein further defines the capsid binding site. Nucleic Acids Res. 1994 Mar;22(5):780-6.

99. Linger BR, Kunovska L, Kuhn RJ, Golden BL. Sindbis virus nucleocapsid assembly: RNA folding promotes capsid protein dimerization. RNA. 2004 Jan;10(1):128-38.

100. Weiss B, Nitschko H, Ghattas I, Wright R, Schlesinger S. Evidence for specificity in the encapsidation of Sindbis virus RNAs. J Virol. 1989 Dec;63(12):5310-8.

101. Gaedigk-Nitschko K, Schlesinger MJ. The Sindbis virus $6 \mathrm{~K}$ protein can be detected in virions and is acylated with fatty acids. Virology. 1990 Mar;175(1):274-81.

102. Metsikko K, Garoff H. Oligomers of the cytoplasmic domain of the p62/E2 membrane protein of Semliki Forest virus bind to the nucleocapsid in vitro. J Virol. 1990 Oct;64(10):4678-83.

103. Lescar J, Roussel A, Wien MW, Navaza J, Fuller SD, Wengler G, et al. The Fusion glycoprotein shell of Semliki Forest virus: an icosahedral assembly primed for fusogenic activation at endosomal pH. Cell. 2001 
Apr;105(1):137-48.

104. Gliedman JB, Smith JF, Brown DT. Morphogenesis of Sindbis virus in cultured Aedes albopictus cells. J Virol. 1975 Oct;16(4):913-26.

105. Lu YE, Kielian M. Semliki forest virus budding: assay, mechanisms, and cholesterol requirement. J Virol. 2000 Sep;74(17):7708-19.

106. Okuda K, Caroci A d. S, Ribolla PEM, de Bianchi AG, Bijovsky AT. Functional morphology of adult female Culex quinquefasciatus midgut during blood digestion. Tissue Cell. 2002;34(3):210-9.

107. Carvalho CAM, Silva JL, Oliveira AC, Gomes AMO. On the entry of an emerging arbovirus into host cells: Mayaro virus takes the highway to the cytoplasm through fusion with early endosomes and caveolae-derived vesicles. PeerJ. 2017;5:e3245.

108. Hoornweg TE, van Duijl-Richter MKS, Ayala Nunez N V, Albulescu IC, van Hemert MJ, Smit JM. Dynamics of Chikungunya Virus Cell Entry Unraveled by Single-Virus Tracking in Living Cells. J Virol. 2016 May;90(9):4745-56.

109. Perrone JB, Spielman A. Time and site of assembly of the peritrophic membrane of the mosquito Aedes aegypti. Cell Tissue Res. 1988 May;252(2):473-8.

110. Kato N, Dasgupta R, Smartt CT, Christensen BM. Glucosamine:fructose-6phosphate aminotransferase: gene characterization, chitin biosynthesis and peritrophic matrix formation in Aedes aegypti. Insect Mol Biol. 2002 Jun;11(3):207-16.

111. Borges-Veloso A, Saboia-Vahia L, Dias-Lopes G, Domont GB, Britto C, Cuervo $P$, et al. In-depth characterization of trypsin-like serine peptidases in the midgut of the sugar fed Culex quinquefasciatus. Parasit Vectors. 2015 Jul 16;8:373.

112. Isoe J, Rascón Jr AA, Kunz S, Miesfeld RL. Molecular genetic analysis of midgut serine proteases in Aedes aegypti mosquitoes. Insect Biochem Mol Biol. 2009/11/03. 2009 Dec;39(12):903-12.

113. Romoser WS. The Biology of Disease Vectors. Beaty BJ, Marquardt WC, editors. Niwot, CO, USA: University Press of Colorado; 1996. 298-317 p.

114. Hecker $\mathrm{H}$. Structure and function of midgut epithelial cells in culicidae mosquitoes (insecta, diptera). Cell Tissue Res. 1977 Nov;184(3):321-41.

115. Houk EJ. Midgut ultrastructure of Culex tarsalis (Diptera:Culcidae) before 
and after a bloodmeal. Tissue Cell. 1977;9(1):103-18.

116. Yurchenco PD, O'Rear JJ. Basal lamina assembly. Curr Opin Cell Biol. 1994 Oct;6(5):674-81.

117. Yurchenco PD, O'Rear JJ. Basement membrane assembly. Methods Enzymol. 1994;245:489-518.

118. Reinhardt $\mathrm{C}$, Hecker $\mathrm{H}$. Structure and function of the basal lamina and of the cell junctions in the midgut epithelium (stomach) of female Aedes aegypti L.(Insecta, Diptera). Acta Trop. 1973;30(4):213-36.

119. Grimstad PR, Walker ED. Aedes triseriatus (Diptera: Culicidae) and La Crosse virus. IV. Nutritional deprivation of larvae affects the adult barriers to infection and transmission. J Med Entomol. 1991 May;28(3):378-86.

120. Thomas RE, Wu WK, Verleye D, Rai KS. Midgut basal lamina thickness and dengue-1 virus dissemination rates in laboratory strains of Aedes albopictus (Diptera: Culicidae). J Med Entomol. 1993 Mar;30(2):326-31.

121. Houk EJ, Hardy JL, Chiles RE. Permeability of the midgut basal lamina in the mosquito, Culex tarsalis Coquillett (Insecta, Diptera). Acta Trop. 1981 Jun;38(2):163-71.

122. Cawston TE, Young DA. Proteinases involved in matrix turnover during cartilage and bone breakdown. Cell Tissue Res. 2010 Jan;339(1):221-35.

123. Seals DF, Courtneidge SA. The ADAMs family of metalloproteases: multidomain proteins with multiple functions. Genes Dev. 2003 Jan;17(1):7-30.

124. Page-McCaw A, Ewald AJ, Werb Z. Matrix metalloproteinases and the regulation of tissue remodelling. Nat Rev Mol Cell Biol. 2007 Mar;8(3):22133.

125. Nagase H, Woessner JFJ. Matrix metalloproteinases. J Biol Chem. 1999 Jul;274(31):21491-4.

126. Vu TH, Werb Z. Matrix metalloproteinases: effectors of development and normal physiology. Genes Dev. 2000 Sep;14(17):2123-33.

127. Rowe RG, Weiss SJ. Breaching the basement membrane: who, when and how? Trends Cell Biol. 2008 Nov;18(11):560-74.

128. Brinckerhoff CE, Matrisian LM. Matrix metalloproteinases: a tail of a frog that became a prince. Nat Rev Mol Cell Biol. 2002 Mar;3(3):207-14. 
129. Browner MF, Smith WW, Castelhano AL. Matrilysin-inhibitor complexes: common themes among metalloproteases. Biochemistry. 1995 May;34(20):6602-10.

130. Marino G, Huesgen PF, Eckhard U, Overall CM, Schroder WP, Funk C. Family-wide characterization of matrix metalloproteinases from Arabidopsis thaliana reveals their distinct proteolytic activity and cleavage site specificity. Biochem J. 2014 Jan;457(2):335-46.

131. Li D, Zhang H, Song Q, Wang L, Liu S, Hong Y, et al. Tomato SI3-MMP, a member of the Matrix metalloproteinase family, is required for disease resistance against Botrytis cinerea and Pseudomonas syringae pv. tomato DC3000. BMC Plant Biol. 2015 Jun;15:143.

132. Wada K, Sato H, Kinoh H, Kajita M, Yamamoto H, Seiki M. Cloning of three Caenorhabditis elegans genes potentially encoding novel matrix metalloproteinases. Gene. 1998 Apr;211(1):57-62.

133. Llano E, Pendas AM, Aza-Blanc P, Kornberg TB, Lopez-Otin C. Dm1MMP, a matrix metalloproteinase from Drosophila with a potential role in extracellular matrix remodeling during neural development. J Biol Chem. 2000 Nov;275(46):35978-85.

134. Llano E, Adam G, Pendas AM, Quesada V, Sanchez LM, Santamaria I, et al. Structural and enzymatic characterization of Drosophila Dm2-MMP, a membrane-bound matrix metalloproteinase with tissue-specific expression. J Biol Chem. 2002 Jun;277(26):23321-9.

135. Page-McCaw A, Serano J, Sante JM, Rubin GM. Drosophila matrix metalloproteinases are required for tissue remodeling, but not embryonic development. Dev Cell. 2003 Jan;4(1):95-106.

136. Page-McCaw A. Remodeling the model organism: matrix metalloproteinase functions in invertebrates. Semin Cell Dev Biol. 2008 Feb;19(1):14-23.

137. Knorr E, Schmidtberg H, Vilcinskas A, Altincicek B. MMPs regulate both development and immunity in the tribolium model insect. PLoS One. 2009;4(3):e4751.

138. Mitten EK, Jing D, Suzuki Y. Matrix metalloproteinases (MMPs) are required for wound closure and healing during larval leg regeneration in the flour beetle, Tribolium castaneum. Insect Biochem Mol Biol. 2012 Nov;42(11):854-64. 
139. Vishnuvardhan S, Ahsan R, Jackson K, Iwanicki R, Boe J, Haring J, et al. Identification of a novel metalloproteinase and its role in juvenile development of the tobacco hornworm, Manduca sexta (Linnaeus). J Exp Zool B Mol Dev Evol. 2013 Mar;320(2):105-17.

140. Goulielmaki E, Siden-Kiamos I, Loukeris TG. Functional characterization of Anopheles matrix metalloprotease 1 reveals its agonistic role during sporogonic development of malaria parasites. Infect Immun. 2014 Nov;82(11):4865-77.

141. Miller CM, Page-McCaw A, Broihier HT. Matrix metalloproteinases promote motor axon fasciculation in the Drosophila embryo. Development. 2008 Jan;135(1):95-109.

142. Bond ND, Nelliot A, Bernardo MK, Ayerh MA, Gorski KA, Hoshizaki DK, et al. ssFTZ-F1 and Matrix metalloproteinase 2 are required for fat-body remodeling in Drosophila. Dev Biol. 2011 Dec;360(2):286-96.

143. Schmidt RL, Rinaldo FM, Hesse SE, Hamada M, Ortiz Z, Beleford DT, et al. Cleavage of PGRP-LC receptor in the Drosophila IMD pathway in response to live bacterial infection in S2 cells. Self Nonself. 2011 Jul;2(3):125-41.

144. Romoser WS, Wasieloski LPJ, Pushko P, Kondig JP, Lerdthusnee K, Neira $\mathrm{M}$, et al. Evidence for arbovirus dissemination conduits from the mosquito (Diptera: Culicidae) midgut. J Med Entomol. 2004 May;41(3):467-75.

145. Engelhard EK, Kam-Morgan LN, Washburn JO, Volkman LE. The insect tracheal system: a conduit for the systemic spread of Autographa californica M nuclear polyhedrosis virus. Proc Natl Acad Sci U S A. 1994 Apr;91(8):3224-7.

146. League GP, Hillyer JF. Functional integration of the circulatory, immune, and respiratory systems in mosquito larvae: pathogen killing in the hemocyte-rich tracheal tufts. BMC Biol. 2016 Sep 19;14:78.

147. Klok CJ. Trachea; Tracheoles: A Fortuitious Discovery Refines a Key Definition. J Exp Biol. 2011 Oct 1;214(19):v LP-v.

148. Bowers DF, Abell BA, Brown DT. Replication and tissue tropism of the alphavirus Sindbis in the mosquito Aedes albopictus. Virology. 1995 Sep;212(1):1-12.

149. Houk EJ, Kramer LD, Hardy JL, Chiles RE. Western equine encephalomyelitis virus: in vivo infection and morphogenesis in mosquito mesenteronal epithelial cells. Virus Res. 1985 Mar;2(2):123-38. 
150. Dong S, Balaraman V, Kantor AM, Lin J, Grant DG, Held NL, et al. Chikungunya virus dissemination from the midgut of Aedes aegypti is associated with temporal basal lamina degradation during bloodmeal digestion. PLoS Negl Trop Dis. 2017 Sep;11(9):e0005976.

151. Romoser WS, Turell MJ, Lerdthusnee K, Neira M, Dohm D, Ludwig G, et al. Pathogenesis of Rift Valley fever virus in mosquitoes-tracheal conduits $\&$ the basal lamina as an extra-cellular barrier. In: Infectious Diseases from Nature: Mechanisms of Viral Emergence and Persistence. Springer; 2005. p. 89-100.

152. Myles KM, Pierro DJ, Olson KE. Comparison of the transmission potential of two genetically distinct Sindbis viruses after oral infection of Aedes aegypti (Diptera: Culicidae). J Med Entomol. 2004 Jan;41(1):95-106.

153. Jupp PG. Culex theileri and Sindbis virus; salivary glands infection in relation to transmission. J Am Mosq Control Assoc. 1985 Sep;1(3):374-6.

154. Turell MJ, Britch SC, Aldridge RL, Kline DL, Boohene C, Linthicum KJ. Potential for mosquitoes (Diptera: Culicidae) from Florida to transmit Rift Valley fever virus. J Med Entomol. 2013 Sep;50(5):1111-7.

155. Blair CD. Mosquito RNAi is the major innate immune pathway controlling arbovirus infection and transmission. Future Microbiol. 2011 Mar;6(3):26577.

156. Kim K, Lee YS, Harris D, Nakahara K, Carthew RW. The RNAi pathway initiated by Dicer-2 in Drosophila. Cold Spring Harb Symp Quant Biol. 2006;71:39-44.

157. Liu X, Jiang F, Kalidas S, Smith D, Liu Q. Dicer-2 and R2D2 coordinately bind siRNA to promote assembly of the siRISC complexes. RNA. 2006 Aug;12(8):1514-20.

158. Okamura K, Ishizuka A, Siomi H, Siomi MC. Distinct roles for Argonaute proteins in small RNA-directed RNA cleavage pathways. Genes Dev. 2004 Jul 15;18(14):1655-66.

159. Gammon DB, Mello CC. RNA interference-mediated antiviral defense in insects. Curr Opin insect Sci. 2015 Apr 1;8:111-20.

160. Blair CD, Olson KE. The role of RNA interference (RNAi) in arbovirusvector interactions. Viruses. 2015 Feb;7(2):820-43.

161. Khoo CCH, Doty JB, Heersink MS, Olson KE, Franz AWE. Transgenemediated suppression of the RNA interference pathway in Aedes aegypti 
interferes with gene silencing and enhances Sindbis virus and dengue virus type 2 replication. Insect Mol Biol. 2013 Feb;22(1):104-14.

162. Sanchez-Vargas I, Scott JC, Poole-Smith BK, Franz AWE, BarbosaSolomieu V, Wilusz J, et al. Dengue virus type 2 infections of Aedes aegypti are modulated by the mosquito's RNA interference pathway. PLoS Pathog. 2009 Feb;5(2):e1000299.

163. Cirimotich CM, Scott JC, Phillips AT, Geiss BJ, Olson KE. Suppression of RNA interference increases alphavirus replication and virus-associated mortality in Aedes aegypti mosquitoes. BMC Microbiol. 2009 Mar;9:49.

164. Myles KM, Wiley MR, Morazzani EM, Adelman ZN. Alphavirus-derived small RNAs modulate pathogenesis in disease vector mosquitoes. Proc Natl Acad Sci U S A. 2008 Dec;105(50):19938-43.

165. Franz AWE, Sanchez-Vargas I, Adelman ZN, Blair CD, Beaty BJ, James $A A$, et al. Engineering RNA interference-based resistance to dengue virus type 2 in genetically modified Aedes aegypti. Proc Natl Acad Sci U S A. 2006 Mar;103(11):4198-203.

166. Weber ANR, Tauszig-Delamasure S, Hoffmann JA, Lelievre E, Gascan H, Ray KP, et al. Binding of the Drosophila cytokine Spatzle to Toll is direct and establishes signaling. Nat Immunol. 2003 Aug;4(8):794-800.

167. Xi Z, Ramirez JL, Dimopoulos G. The Aedes aegypti toll pathway controls dengue virus infection. PLoS Pathog. 2008 Jul;4(7):e1000098.

168. Colpitts TM, Cox J, Vanlandingham DL, Feitosa FM, Cheng G, Kurscheid $\mathrm{S}$, et al. Alterations in the Aedes aegypti transcriptome during infection with West Nile, dengue and yellow fever viruses. PLoS Pathog. 2011 Sep;7(9):e1002189.

169. Luplertlop N, Surasombatpattana P, Patramool S, Dumas E, Wasinpiyamongkol L, Saune L, et al. Induction of a peptide with activity against a broad spectrum of pathogens in the Aedes aegypti salivary gland, following Infection with Dengue Virus. PLoS Pathog. 2011 Jan;7(1):e1001252.

170. Sim S, Jupatanakul N, Dimopoulos G. Mosquito immunity against arboviruses. Viruses. 2014 Nov 19;6(11):4479-504.

171. Pan X, Zhou G, Wu J, Bian G, Lu P, Raikhel AS, et al. Wolbachia induces reactive oxygen species (ROS)-dependent activation of the Toll pathway to control dengue virus in the mosquito Aedes aegypti. Proc Natl Acad Sci U S A. 2012 Jan;109(1):E23-31. 
172. Moreira LA, Iturbe-Ormaetxe I, Jeffery JA, Lu G, Pyke AT, Hedges LM, et al. A Wolbachia symbiont in Aedes aegypti limits infection with dengue, Chikungunya, and Plasmodium. Cell. 2009 Dec;139(7):1268-78.

173. Bian G, Xu Y, Lu P, Xie Y, Xi Z. The endosymbiotic bacterium Wolbachia induces resistance to dengue virus in Aedes aegypti. PLoS Pathog. 2010 Apr;6(4):e1000833.

174. Sanders HR, Foy BD, Evans AM, Ross LS, Beaty BJ, Olson KE, et al. Sindbis virus induces transport processes and alters expression of innate immunity pathway genes in the midgut of the disease vector, Aedes aegypti. Insect Biochem Mol Biol. 2005 Nov;35(11):1293-307.

175. Fragkoudis R, Chi Y, Siu RWC, Barry G, Attarzadeh-Yazdi G, Merits A, et al. Semliki Forest virus strongly reduces mosquito host defence signaling. Insect Mol Biol. 2008 Dec;17(6):647-56.

176. Avadhanula V, Weasner BP, Hardy GG, Kumar JP, Hardy RW. A novel system for the launch of alphavirus RNA synthesis reveals a role for the Imd pathway in arthropod antiviral response. PLoS Pathog. 2009 Sep;5(9):e1000582.

177. Sim S, Jupatanakul N, Ramirez JL, Kang S, Romero-Vivas CM, Mohammed $\mathrm{H}$, et al. Transcriptomic profiling of diverse Aedes aegypti strains reveals increased basal-level immune activation in dengue virusrefractory populations and identifies novel virus-vector molecular interactions. PLoS Negl Trop Dis. 2013;7(7):e2295.

178. Arbouzova NI, Zeidler MP. JAK/STAT signalling in Drosophila: insights into conserved regulatory and cellular functions. Development. 2006 Jul;133(14):2605-16.

179. Souza-Neto JA, Sim S, Dimopoulos G. An evolutionary conserved function of the JAK-STAT pathway in anti-dengue defense. Proc Natl Acad Sci U S A. 2009 Oct;106(42):17841-6.

180. Waldock J, Olson KE, Christophides GK. Anopheles gambiae antiviral immune response to systemic O'nyong-nyong infection. PLoS Negl Trop Dis. 2012;6(3):e1565.

181. Notredame C, Higgins DG, Heringa J. T-Coffee: A novel method for fast and accurate multiple sequence alignment. J Mol Biol. 2000 Sep;302(1):205-17.

182. Hasegawa M, Kishino H, Yano T. Dating of the human-ape splitting by a molecular clock of mitochondrial DNA. J Mol Evol. 1985;22(2):160-74. 
183. Guindon S, Gascuel O. A simple, fast, and accurate algorithm to estimate large phylogenies by maximum likelihood. Syst Biol. 2003 Oct;52(5):696704.

184. Livak KJ, Schmittgen TD. Analysis of relative gene expression data using real-time quantitative PCR and the 2(-Delta Delta C(T)) Method. Methods. $2001 \mathrm{Dec} ; 25(4): 402-8$.

185. Deerinck TJ, Bushong E, Thor A, Ellisman MH. NCMIR Methods for 3D EM: A New Protocol for Preparation of Biological Specimens for Serial Block-Face SEM. SBEM Protocol v7_01_2010. 2018.

186. Villinger C, Schauflinger M, Gregorius H, Kranz C, Hohn K, Nafeey S, et al. Three-dimensional imaging of adherent cells using FIB/SEM and STEM. Methods Mol Biol. 2014;1117:617-38.

187. Weaver SC, Reisen WK. Present and future arboviral threats. Antiviral Res. 2010 Feb;85(2):328-45.

188. Weaver SC. Urbanization and geographic expansion of zoonotic arboviral diseases: mechanisms and potential strategies for prevention. Trends Microbiol. 2013 Aug;21(8):360-3.

189. Franz AWE, Kantor AM, Passarelli AL, Clem RJ. Tissue Barriers to Arbovirus Infection in Mosquitoes. Viruses. 2015 Jul;7(7):3741-67.

190. Means JC, Passarelli AL. Viral fibroblast growth factor, matrix metalloproteases, and caspases are associated with enhancing systemic infection by baculoviruses. Proc Natl Acad Sci U S A. 2010 May;107(21):9825-30.

191. Passarelli AL. Barriers to success: how baculoviruses establish efficient systemic infections. Virology. 2011 Mar;411(2):383-92.

192. Massova I, Kotra LP, Fridman R, Mobashery S. Matrix metalloproteinases: structures, evolution, and diversification. FASEB J Off Publ Fed Am Soc Exp Biol. 1998 Sep;12(12):1075-95.

193. Lopez-Pelegrin M, Ksiazek M, Karim AY, Guevara T, Arolas JL, Potempa $\mathrm{J}$, et al. A novel mechanism of latency in matrix metalloproteinases. J Biol Chem. 2015 Feb;290(8):4728-40.

194. Bode W, Gomis-Ruth FX, Stockler W. Astacins, serralysins, snake venom and matrix metalloproteinases exhibit identical zinc-binding environments (HEXXHXXGXXH and Met-turn) and topologies and should be grouped into a common family, the "metzincins". FEBS Lett. 1993 Sep;331(1- 
2):134-40.

195. Fasciglione GF, Gioia M, Tsukada H, Liang J, lundusi R, Tarantino U, et al. The collagenolytic action of MMP-1 is regulated by the interaction between the catalytic domain and the hinge region. J Biol Inorg Chem. 2012 Apr;17(4):663-72.

196. Brew K, Dinakarpandian D, Nagase H. Tissue inhibitors of metalloproteinases: evolution, structure and function. Biochim Biophys Acta. 2000 Mar;1477(1-2):267-83.

197. Overall CM. Molecular determinants of metalloproteinase substrate specificity: matrix metalloproteinase substrate binding domains, modules, and exosites. Mol Biotechnol. 2002 Sep;22(1):51-86.

198. Mott JD, Werb Z. Regulation of matrix biology by matrix metalloproteinases. Curr Opin Cell Biol. 2004 Oct;16(5):558-64.

199. Jackson BC, Nebert DW, Vasiliou V. Update of human and mouse matrix metalloproteinase families. Hum Genomics. 2010 Feb;4(3):194-201.

200. Lohi J, Wilson CL, Roby JD, Parks WC. Epilysin, a novel human matrix metalloproteinase (MMP-28) expressed in testis and keratinocytes and in response to injury. J Biol Chem. 2001 Mar;276(13):10134-44.

201. Neumann U, Kubota H, Frei K, Ganu V, Leppert D. Characterization of Mca-Lys-Pro-Leu-Gly-Leu-Dpa-Ala-Arg-NH2, a fluorogenic substrate with increased specificity constants for collagenases and tumor necrosis factor converting enzyme. Anal Biochem. 2004 May;328(2):166-73.

202. Sternlicht MD, Werb Z. How matrix metalloproteinases regulate cell behavior. Annu Rev Cell Dev Biol. 2001;17:463-516.

203. Gomez DE, Alonso DF, Yoshiji H, Thorgeirsson UP. Tissue inhibitors of metalloproteinases: structure, regulation and biological functions. Eur $\mathrm{J}$ Cell Biol. 1997 Oct;74(2):111-22.

204. Ra H-J, Parks WC. Control of matrix metalloproteinase catalytic activity. Matrix Biol. 2007 Oct;26(8):587-96.

205. Bonizzoni M, Dunn WA, Campbell CL, Olson KE, Marinotti O, James AA. Complex modulation of the Aedes aegypti transcriptome in response to dengue virus infection. PLoS One. 2012;7(11):e50512.

206. Srivastava A, Pastor-Pareja JC, Igaki T, Pagliarini R, Xu T. Basement membrane remodeling is essential for Drosophila disc eversion and tumor 
invasion. Proc Natl Acad Sci U S A. 2007 Feb;104(8):2721-6.

207. Glasheen BM, Kabra AT, Page-McCaw A. Distinct functions for the catalytic and hemopexin domains of a Drosophila matrix metalloproteinase. Proc Natl Acad Sci U S A. 2009 Feb;106(8):2659-64.

208. Stevens LJ, Page-McCaw A. A secreted MMP is required for reepithelialization during wound healing. Mol Biol Cell. 2012 Mar;23(6):1068-79.

209. Powers AM. Chikungunya. Clin Lab Med. 2010 Mar;30(1):209-19.

210. Fischer M, Staples JE. Notes from the field: chikungunya virus spreads in the Americas - Caribbean and South America, 2013-2014. MMWR Morb Mortal Wkly Rep. 2014 Jun;63(22):500-1.

211. Morrison TE. Reemergence of chikungunya virus. J Virol. 2014 Oct;88(20):11644-7.

212. Solignat M, Gay B, Higgs S, Briant L, Devaux C. Replication cycle of chikungunya: a re-emerging arbovirus. Virology. 2009 Oct;393(2):183-97.

213. Hecker H, Rudin W. Morphometric parameters of the midgut cells of Aedes aegypti L. (Insecta, Diptera) under various conditions. Cell Tissue Res. $1981 ; 219(3): 619-27$.

214. Hardy JL, Houk EJ, Kramer LD, Reeves WC. Intrinsic factors affecting vector competence of mosquitoes for arboviruses. Annu Rev Entomol. 1983;28:229-62.

215. Whitfield SG, Murphy FA, Sudia WD. St. Louis encephalitis virus: An ultrastructural study of infection in a mosquito vector. Virology. 1973;56(1):70-87.

216. Weaver SC. Electron microscopic analysis of infection patterns for Venezuelan equine encephalomyelitis virus in the vector mosquito, Culex (Melanoconion) taeniopus. Am J Trop Med Hyg. 1986 May;35(3):624-31.

217. Lerdthusnee K, Romoser WS, Faran ME, Dohm DJ. Rift Valley fever virus in the cardia of Culex pipiens: an immunocytochemical and ultrastructural study. Am J Trop Med Hyg. 1995 Oct;53(4):331-7.

218. Girard YA, Popov V, Wen J, Han V, Higgs S. Ultrastructural study of West Nile virus pathogenesis in Culex pipiens quinquefasciatus (Diptera: Culicidae). J Med Entomol. 2005 May;42(3):429-44. 
219. Hernandez R, Brown DT, Paredes A. Structural differences observed in arboviruses of the alphavirus and flavivirus genera. Adv Virol. 2014;2014:259382.

220. Fernández-de-Castro I, Risco C. Imaging RNA virus replication assemblies: bunyaviruses and reoviruses. Future Virol. 2014;9(12):1089104.

221. Scott TW, Hildreth SW, Beaty BJ. The distribution and development of eastern equine encephalitis virus in its enzootic mosquito vector, Culiseta melanura. Am J Trop Med Hyg. 1984 Mar;33(2):300-10.

222. Larsen JR, Ashley RF. Demonstration of Venezuelan equine encephalomyelitis virus in tissues of Aedes Aegypti. Am J Trop Med Hyg. 1971 Sep;20(5):754-60.

223. Scott TW, Burrage TG. Rapid infection of salivary glands in Culiseta melanura with eastern equine encephalitis virus: an electron microscopic study. Am J Trop Med Hyg. 1984 Sep;33(5):961-4.

224. Weaver SC, Scott TW, Lorenz LH, Lerdthusnee K, Romoser WS. Togavirus-associated pathologic changes in the midgut of a natural mosquito vector. J Virol. 1988 Jun;62(6):2083-90.

225. Weaver SC, Lorenz LH, Scott TW. Pathologic changes in the midgut of Culex tarsalis following infection with Western equine encephalomyelitis virus. Am J Trop Med Hyg. 1992 Nov;47(5):691-701.

226. Vega-Rua A, Schmitt C, Bonne I, Krijnse Locker J, Failloux A-B. Chikungunya Virus Replication in Salivary Glands of the Mosquito Aedes albopictus. Viruses. 2015 Nov;7(11):5902-7.

227. Soekiman S, Matsumura T, Yamanishi H. Multiplication of chikungunya virus in salivary glands of Aedes albopictus (Oahu strain) mosquitoes: an electron microscopic study. Jpn J Med Sci Biol. 1986;39(5-6):207-11.

228. Janzen HG, Rhodes AJ, Doane FW. Chikungunya virus in salivary glands of Aedes aegypti (L.): an electron microscope study. Can J Microbiol. 1970 Jul;16(7):581-6.

229. Smith DR, Arrigo NC, Leal G, Muehlberger LE, Weaver SC. Infection and dissemination of Venezuelan equine encephalitis virus in the epidemic mosquito vector, Aedes taeniorhynchus. Am J Trop Med Hyg. 2007 Jul;77(1):176-87.

230. Romoser WS, Faran ME, Bailey CL. Newly recognized route of arbovirus 
dissemination from the mosquito (Diptera: Culicidae) midgut. J Med Entomol. 1987 Jul;24(4):431-2.

231. Romoser WS, Faran ME, Bailey CL, Lerdthusnee K. An immunocytochemical study of the distribution of Rift Valley fever virus in the mosquito Culex pipiens. Am J Trop Med Hyg. 1992 Apr;46(4):489-501.

232. Foy BD, Myles KM, Pierro DJ, Sanchez-Vargas I, Uhlirova M, Jindra M, et al. Development of a new Sindbis virus transducing system and its characterization in three Culicine mosquitoes and two Lepidopteran species. Insect Mol Biol. 2004 Feb;13(1):89-100.

233. Weaver SC, Scott TW, Lorenz LH, Repik PM. Detection of eastern equine encephalomyelitis virus deposition in Culiseta melanura following ingestion of radiolabeled virus in blood meals. Am J Trop Med Hyg. 1991 Mar;44(3):250-9.

234. Brault AC, Foy BD, Myles KM, Kelly CLH, Higgs S, Weaver SC, et al. Infection patterns of o'nyong nyong virus in the malaria-transmitting mosquito, Anopheles gambiae. Insect Mol Biol. 2004 Dec;13(6):625-35.

235. Salazar MI, Richardson JH, Sanchez-Vargas I, Olson KE, Beaty BJ. Dengue virus type 2: replication and tropisms in orally infected Aedes aegypti mosquitoes. BMC Microbiol. 2007 Jan; 7:9.

236. Kramer LD, Hardy JL, Presser SB, Houk EJ. Dissemination barriers for western equine encephalomyelitis virus in Culex tarsalis infected after ingestion of low viral doses. Am J Trop Med Hyg. 1981 Jan;30(1):190-7.

237. Bennett KE, Flick D, Fleming KH, Jochim R, Beaty BJ, Black WC 4th. Quantitative trait loci that control dengue-2 virus dissemination in the mosquito Aedes aegypti. Genetics. 2005 May;170(1):185-94.

238. Bennett KE, Beaty BJ, Black WC 4th. Selection of D2S3, an Aedes aegypti (Diptera: Culicidae) strain with high oral susceptibility to Dengue 2 virus and D2MEB, a strain with a midgut barrier to Dengue 2 escape. J Med Entomol. 2005 Mar;42(2):110-9.

239. Khoo $\mathrm{CCH}$, Doty JB, Held NL, Olson KE, Franz AWE. Isolation of midgut escape mutants of two American genotype dengue 2 viruses from Aedes aegypti. Virol J. 2013 Aug;10:257.

240. Dong S, Behura SK, Franz AWE. The midgut transcriptome of Aedes aegypti fed with saline or protein meals containing chikungunya virus reveals genes potentially involved in viral midgut escape. BMC Genomics. 2017 May;18(1):382. 
241. Hardy JL. Susceptibility and resistance of vector mosquitoes. arboviruses Epidemiol Ecol. 1988;1:87-126.

242. Reddy JT, Locke M. The size limited penetration of gold particles through insect basal laminae. J Insect Physiol. 1990;36(6):397-407.

243. Kantor AM, Dong S, Held NL, Ishimwe E, Passarelli AL, Clem RJ, et al. Identification and initial characterization of matrix metalloproteinases in the yellow fever mosquito, Aedes aegypti. Insect Mol Biol. 2017 Feb;26(1):113-26.

244. Drobne D, Milani M, Zrimec A, Zrimec MB, Tatti F, Draslar K. Focused ion beam/scanning electron microscopy studies of Porcellio scaber (Isopoda, Crustacea) digestive gland epithelium cells. Scanning. 2005;27(1):30-4.

245. Heymann JAW, Hayles M, Gestmann I, Giannuzzi LA, Lich B, Subramaniam S. Site-specific 3D imaging of cells and tissues with a dual beam microscope. J Struct Biol. 2006 Jul;155(1):63-73.

246. Carpenter KJ, Weber PK, Davisson ML, Pett-Ridge J, Haverty MI, Keeling PJ. Correlated SEM, FIB-SEM, TEM, and NanoSIMS imaging of microbes from the hindgut of a lower termite: methods for in situ functional and ecological studies of uncultivable microbes. Microsc Microanal. 2013 Dec;19(6):1490-501.

247. Kizilyaprak C, Daraspe J, Humbel BM. Focused ion beam scanning electron microscopy in biology. J Microsc. 2014 Jun;254(3):109-14.

248. Di Giulio A, Muzzi M. Two novel approaches to study arthropod anatomy by using dualbeam FIB/SEM. Micron. 2018 Mar;106:21-6.

249. Auguste AJ, Liria J, Forrester NL, Giambalvo D, Moncada M, Long KC, et al. Evolutionary and Ecological Characterization of Mayaro Virus Strains Isolated during an Outbreak, Venezuela, 2010. Emerg Infect Dis. 2015 Oct;21(10):1742-50.

250. Yao JS, Strauss EG, Strauss JH. Interactions between PE2, E1, and 6K required for assembly of alphaviruses studied with chimeric viruses. J Virol. 1996 Nov;70(11):7910-20.

251. Lanciotti RS, Kosoy OL, Laven JJ, Panella AJ, Velez JO, Lambert AJ, et al. Chikungunya virus in US travelers returning from India, 2006. Emerg Infect Dis. 2007 May;13(5):764-7.

252. Mukhopadhyay S, Chipman PR, Hong EM, Kuhn RJ, Rossmann MG. In vitro-assembled alphavirus core-like particles maintain a structure similar to 
that of nucleocapsid cores in mature virus. J Virol. 2002 Nov;76(21):11128-32.

253. Farjana T, Tuno N. Multiple blood feeding and host-seeking behavior in Aedes aegypti and Aedes albopictus (Diptera: Culicidae). J Med Entomol. 2013 Jul;50(4):838-46.

254. Weaver SC, Scott TW, Lorenz LH. Patterns of eastern equine encephalomyelitis virus infection in Culiseta melanura (Diptera: Culicidae). J Med Entomol. 1990 Sep;27(5):878-91.

255. Vaidyanathan R, Scott TW. Apoptosis in mosquito midgut epithelia associated with West Nile virus infection. Apoptosis. 2006 Sep;11(9):164351.

256. Wang $\mathrm{H}$, Gort $\mathrm{T}$, Boyle DL, Clem RJ. Effects of manipulating apoptosis on Sindbis virus infection of Aedes aegypti mosquitoes. J Virol. 2012 Jun;86(12):6546-54.

257. Liu Q, Clem RJ. Defining the core apoptosis pathway in the mosquito disease vector Aedes aegypti: the roles of iap1, ark, dronc, and effector caspases. Apoptosis. 2011 Feb;16(2):105-13.

258. O'Neill K, Olson BJSC, Huang N, Unis D, Clem RJ. Rapid selection against arbovirus-induced apoptosis during infection of a mosquito vector. Proc Natl Acad Sci U S A. 2015 Mar;112(10):E1152-61.

259. Casals J, Whitman L. Mayaro virus: a new human disease agent. I. Relationship to other arbor viruses. Am J Trop Med Hyg. 1957 Nov;6(6):1004-11.

260. Causey OR, Maroja OM. Mayaro virus: a new human disease agent. III. Investigation of an epidemic of acute febrile illness on the river Guama in Para, Brazil, and isolation of Mayaro virus as causative agent. Am J Trop Med Hyg. 1957 Nov;6(6):1017-23.

261. Aitken TH, Downs WG, Anderson CR, Spence L, Casals J. Mayaro virus isolated from a Trinidadian mosquito, Mansonia venezuelensis. Science. 1960 Apr;131(3405):986.

262. Izurieta RO, Macaluso M, Watts DM, Tesh RB, Guerra B, Cruz LM, et al. Hunting in the Rainforest and Mayaro Virus Infection: An emerging Alphavirus in Ecuador. J Glob Infect Dis. 2011 Oct;3(4):317-23.

263. Calisher $\mathrm{CH}$, Gutierrez E, Maness KS, Lord RD. Isolation of Mayaro virus from a migrating bird captured in Louisiana in 1967. Bull Pan Am Health 
Organ. 1974;8(3):243-8.

264. de Thoisy B, Gardon J, Salas RA, Morvan J, Kazanji M. Mayaro virus in wild mammals, French Guiana. Emerg Infect Dis. 2003 Oct;9(10):1326-9.

265. Pauvolid-Correa A, Juliano RS, Campos Z, Velez J, Nogueira RMR, Komar N. Neutralising antibodies for Mayaro virus in Pantanal, Brazil. Mem Inst Oswaldo Cruz. 2015 Feb;110(1):125-33.

266. Tesh RB, Watts DM, Russell KL, Damodaran C, Calampa C, Cabezas C, et al. Mayaro virus disease: an emerging mosquito-borne zoonosis in tropical South America. Clin Infect Dis. 1999 Jan;28(1):67-73.

267. Powers AM, Aguilar P V, Chandler LJ, Brault AC, Meakins TA, Watts D, et al. Genetic relationships among Mayaro and Una viruses suggest distinct patterns of transmission. Am J Trop Med Hyg. 2006 Sep;75(3):461-9.

268. Smith GC, Francy DB. Laboratory studies of a Brazilian strain of Aedes albopictus as a potential vector of Mayaro and Oropouche viruses. J Am Mosq Control Assoc. 1991 Mar;7(1):89-93.

269. Wiggins K, Eastmond B, Alto BW. Transmission potential of Mayaro virus in Florida Aedes aegypti and Aedes albopictus mosquitoes. Med Vet Entomol. 2018 Dec;32(4):436-42.

270. Kantor AM, Grant DG, Balaraman V, White TA, Franz AWE. Ultrastructural Analysis of Chikungunya Virus Dissemination from the Midgut of the Yellow Fever Mosquito, Aedes aegypti. Viruses. 2018 Oct;10(10).

271. Dupont-Rouzeyrol M, O'Connor O, Calvez E, Daures M, John M, Grangeon J-P, et al. Co-infection with Zika and dengue viruses in 2 patients, New Caledonia, 2014. Vol. 21, Emerging infectious diseases. United States; 2015. p. 381-2.

272. Furuya-Kanamori L, Liang S, Milinovich G, Soares Magalhaes RJ, Clements ACA, Hu W, et al. Co-distribution and co-infection of chikungunya and dengue viruses. BMC Infect Dis. 2016 Mar;16:84.

273. Villamil-Gomez WE, Rodriguez-Morales AJ, Uribe-Garcia AM, GonzalezArismendy E, Castellanos JE, Calvo EP, et al. Zika, dengue, and chikungunya co-infection in a pregnant woman from Colombia. Int J Infect Dis. 2016 Oct;51:135-8.

274. Waggoner JJ, Gresh L, Vargas MJ, Ballesteros G, Tellez Y, Soda KJ, et al. Viremia and Clinical Presentation in Nicaraguan Patients Infected With Zika Virus, Chikungunya Virus, and Dengue Virus. Clin Infect Dis. 2016 
Dec;63(12):1584-90.

275. Zambrano H, Waggoner JJ, Almeida C, Rivera L, Benjamin JQ, Pinsky BA. Zika Virus and Chikungunya Virus Colnfections: A Series of Three Cases from a Single Center in Ecuador. Am J Trop Med Hyg. 2016 Oct;95(4):894-6.

276. Dubrulle M, Mousson L, Moutailler S, Vazeille M, Failloux A-B. Chikungunya virus and Aedes mosquitoes: saliva is infectious as soon as two days after oral infection. PLoS One. 2009 Jun;4(6):e5895.

277. Gaidamovich SY, Khutoretskaya N V, Lvova AI, Sveshnikova NA. Immunofluorescent staining study of the salivary glands of mosquitoes infected with group A arboviruses. Intervirology. 1973;1(3):193-200

278. Faran ME, Romoser WS, Routier RG, Bailey CL. The distribution of Rift Valley fever virus in the mosquito Culex pipiens as revealed by viral titration of dissected organs and tissues. Am J Trop Med Hyg. 1988 Aug;39(2):206-13.

279. Vazeille M, Mousson L, Martin E, Failloux A-B. Orally co-Infected Aedes albopictus from La Reunion Island, Indian Ocean, can deliver both dengue and chikungunya infectious viral particles in their saliva. PLoS Negl Trop Dis. 2010 Jun;4(6):e706.

280. Rückert C, Weger-Lucarelli J, Garcia-Luna SM, Young MC, Byas AD, Murrieta RA, et al. Impact of simultaneous exposure to arboviruses on infection and transmission by Aedes aegypti mosquitoes. Nat Commun. 2017 May 19;8:15412.

281. Myers RM, Carey DE. Concurrent isolation from patient of two arboviruses, Chikungunya and dengue type 2. Science. 1967 Sep;157(3794):1307-8.

282. Condreay LD, Brown DT. Exclusion of superinfecting homologous virus by Sindbis virus-infected Aedes albopictus (mosquito) cells. J Virol. 1986 Apr;58(1):81-6.

283. Karpf AR, Lenches E, Strauss EG, Strauss JH, Brown DT. Superinfection exclusion of alphaviruses in three mosquito cell lines persistently infected with Sindbis virus. J Virol. 1997 Sep;71(9):7119-23.

284. Abrao EP, da Fonseca BAL. Infection of Mosquito Cells (C6/36) by Dengue-2 Virus Interferes with Subsequent Infection by Yellow Fever Virus. Vector Borne Zoonotic Dis. 2016 Feb;16(2):124-30.

285. Sundin DR, Beaty BJ. Interference to oral superinfection of Aedes 
triseriatus infected with La Crosse virus. Am J Trop Med Hyg. 1988 Mar;38(2):428-32.

286. Borucki MK, Chandler LJ, Parker BM, Blair CD, Beaty BJ. Bunyavirus superinfection and segment reassortment in transovarially infected mosquitoes. J Gen Virol. 1999 Dec;80 Pt 12:3173-9.

287. Pesko K, Mores CN. Effect of sequential exposure on infection and dissemination rates for West Nile and St. Louis encephalitis viruses in Culex quinquefasciatus. Vector Borne Zoonotic Dis. 2009 Jun;9(3):281-6.

288. Campbell CL, Smith DR, Sanchez-Vargas I, Zhang B, Shi P-Y, Ebel GD. A positively selected mutation in the WNV $2 \mathrm{~K}$ peptide confers resistance to superinfection exclusion in vivo. Virology. 2014 Sep;464-465:228-32.

289. Nasar F, Erasmus JH, Haddow AD, Tesh RB, Weaver SC. Eilat virus induces both homologous and heterologous interference. Virology. 2015 Oct;484:51-8.

290. Nuckols JT, Huang Y-JS, Higgs S, Miller AL, Pyles RB, Spratt HM, et al. Evaluation of Simultaneous Transmission of Chikungunya Virus and Dengue Virus Type 2 in Infected Aedes aegypti and Aedes albopictus (Diptera: Culicidae). J Med Entomol. 2015 May;52(3):447-51.

291. Kent RJ, Crabtree MB, Miller BR. Transmission of West Nile virus by Culex quinquefasciatus say infected with Culex Flavivirus Izabal. PLoS Negl Trop Dis. 2010 May;4(5):e671.

292. Bolling BG, Olea-Popelka FJ, Eisen L, Moore CG, Blair CD. Transmission dynamics of an insect-specific flavivirus in a naturally infected Culex pipiens laboratory colony and effects of co-infection on vector competence for West Nile virus. Virology. 2012 Jun;427(2):90-7.

293. Goenaga S, Kenney JL, Duggal NK, Delorey M, Ebel GD, Zhang B, et al. Potential for Co-Infection of a Mosquito-Specific Flavivirus, Nhumirim Virus, to Block West Nile Virus Transmission in Mosquitoes. Viruses. 2015 Nov;7(11):5801-12.

294. Singh IR, Suomalainen M, Varadarajan S, Garoff H, Helenius A. Multiple mechanisms for the inhibition of entry and uncoating of superinfecting Semliki Forest virus. Virology. 1997 Apr;231(1):59-71.

295. Edwards MJ, Moskalyk LA, Donelly-Doman M, Vlaskova M, Noriega FG, Walker VK, et al. Characterization of a carboxypeptidase A gene from the mosquito, Aedes aegypti. Insect Mol Biol. 2000 Feb;9(1):33-8. 
296. Brew K, Nagase H. The tissue inhibitors of metalloproteinases (TIMPs): an ancient family with structural and functional diversity. Biochim Biophys Acta. 2010 Jan;1803(1):55-71.

297. Yu WH, Yu S, Meng Q, Brew K, Woessner JFJ. TIMP-3 binds to sulfated glycosaminoglycans of the extracellular matrix. J Biol Chem. 2000 Oct;275(40):31226-32.

298. Wang T, Yamashita K, Iwata K, Hayakawa T. Both tissue inhibitors of metalloproteinases-1 (TIMP-1) and TIMP-2 activate Ras but through different pathways. Biochem Biophys Res Commun. 2002 Aug;296(1):2015 .

299. Edwards DR, Handsley MM, Pennington CJ. The ADAM metalloproteinases. Mol Aspects Med. 2008 Oct;29(5):258-89.

300. Albrecht S, Wang S, Holz A, Bergter A, Paululat A. The ADAM metalloprotease Kuzbanian is crucial for proper heart formation in Drosophila melanogaster. Mech Dev. 2006 May;123(5):372-87.

301. Herzog C, Haun RS, Ludwig A, Shah S V, Kaushal GP. ADAM10 is the major sheddase responsible for the release of membrane-associated meprin A. J Biol Chem. 2014 May;289(19):13308-22.

302. Giebeler N, Zigrino P. A Disintegrin and Metalloprotease (ADAM): Historical Overview of Their Functions. Toxins (Basel). 2016 Apr 23;8(4):122.

303. Millichip MI, Dallas DJ, Wu E, Dale S, McKie N. The metallo-disintegrin ADAM10 (MADM) from bovine kidney has type IV collagenase activity in vitro. Biochem Biophys Res Commun. 1998 Apr;245(2):594-8.

304. Kelwick R, Desanlis I, Wheeler GN, Edwards DR. The ADAMTS (A Disintegrin and Metalloproteinase with Thrombospondin motifs) family. Genome Biol. 2015 May 30;16(1):113.

305. Bekhouche M, Colige A. The procollagen N-proteinases ADAMTS2, 3 and 14 in pathophysiology. Matrix Biol. 2015;44-46:46-53.

306. Nandadasa S, Foulcer S, Apte SS. The multiple, complex roles of versican and its proteolytic turnover by ADAMTS proteases during embryogenesis. Matrix Biol. 2014 Apr;35:34-41.

307. Kim G-W, Won J-H, Lee O-K, Lee S-S, Han J-H, Tsogtbaatar O, et al. Sol narae (Sona) is a Drosophila ADAMTS involved in Wg signaling. Sci Rep. 2016 Aug;6:31863. 
308. Barreau C, Conrad J, Fischer E, Lujan HD, Vernick KD. Identification of surface molecules on salivary glands of the mosquito, Aedes aegypti, by a panel of monoclonal antibodies. Insect Biochem Mol Biol. 1999 Jun;29(6):515-26.

309. Perrone JB, DeMaio J, Spielman A. Regions of mosquito salivary glands distinguished by surface lectin-binding characteristics. Insect Biochem. 1986;16(2):313-8.

310. Lee C-Y, Clough EA, Yellon P, Teslovich TM, Stephan DA, Baehrecke EH. Genome-wide analyses of steroid- and radiation-triggered programmed cell death in Drosophila. Curr Biol. 2003 Feb;13(4):350-7.

311. Oviedo MVN, Romoser WS, James CB, Mahmood F, Reisen WK. Infection dynamics of western equine encephalomyelitis virus (Togaviridae: Alphavirus) in four strains of Culex tarsalis (Diptera: Culicidae): an immunocytochemical study. Res Rep Trop Med. 2011 Apr;2011(2):65-77.

312. Ahmad NA, Vythilingam I, Lim YAL, Zabari NZAM, Lee HL. Detection of Wolbachia in Aedes albopictus and Their Effects on Chikungunya Virus. Am J Trop Med Hyg. 2017 Jan 11;96(1):148-56.

313. King JG, Souto-Maior C, Sartori LM, Maciel-de-Freitas R, Gomes MGM. Variation in Wolbachia effects on Aedes mosquitoes as a determinant of invasiveness and vectorial capacity. Nat Commun. 2018 Apr;9(1):1483.

314. Munoz M, Navarro JC. Mayaro: a re-emerging Arbovirus in Venezuela and Latin America. Biomedica. 2012 Jun;32(2):286-302.

315. Le Coupanec A, Tchankouo-Nguetcheu S, Roux P, Khun H, Huerre M, Morales-Vargas R, et al. Co-Infection of Mosquitoes with Chikungunya and Dengue Viruses Reveals Modulation of the Replication of Both Viruses in Midguts and Salivary Glands of Aedes aegypti Mosquitoes. Int J Mol Sci. 2017 Aug;18(8).

316. Kummerer BM, Grywna K, Glasker S, Wieseler J, Drosten C. Construction of an infectious Chikungunya virus cDNA clone and stable insertion of mCherry reporter genes at two different sites. J Gen Virol. 2012 Sep;93(Pt 9):1991-5.

317. Thomas JM, Klimstra WB, Ryman KD, Heidner HW. Sindbis virus vectors designed to express a foreign protein as a cleavable component of the viral structural polyprotein. J Virol. 2003 May;77(10):5598-606.

318. Tamberg N, Lulla V, Fragkoudis R, Lulla A, Fazakerley JK, Merits A. Insertion of EGFP into the replicase gene of Semliki Forest virus results in 
a novel, genetically stable marker virus. J Gen Virol. 2007 Apr;88(Pt 4):1225-30.

319. Vanlandingham DL, Tsetsarkin K, Hong C, Klingler K, McElroy KL, Lehane $\mathrm{MJ}$, et al. Development and characterization of a double subgenomic chikungunya virus infectious clone to express heterologous genes in Aedes aegypti mosqutioes. Insect Biochem Mol Biol. 2005;35(10):1162-70.

320. Foy BD, Olson KE. Alphavirus transducing systems. Adv Exp Med Biol. 2008;627:19-34.

321. Pierro DJ, Myles KM, Foy BD, Beaty BJ, Olson KE. Development of an orally infectious Sindbis virus transducing system that efficiently disseminates and expresses green fluorescent protein in Aedes aegypti. Insect Mol Biol. 2003 Apr;12(2):107-16.

322. Phillips A, Mossel E, Sanchez-Vargas I, Foy B, Olson K. Alphavirus transducing system: tools for visualizing infection in mosquito vectors. $J$ Vis Exp. $2010 \mathrm{Nov} ;(45)$.

323. Olson KE, Myles KM, Seabaugh RC, Higgs S, Carlson JO, Beaty BJ. Development of a Sindbis virus expression system that efficiently expresses green fluorescent protein in midguts of Aedes aegypti following per os infection. Insect Mol Biol. 2000 Feb;9(1):57-65. 


\section{Vita}

Asher Michael Kantor was born to Barry and Cheryl Kantor on August $7^{\text {th }}, 1991$ in Overland Park Kansas. He was raised there alongside his older brother, Josh Kantor. Asher graduated from Blue Valley Northwest Highschool in 2009, and completed his undergraduate work at the University of Missouri where he received a Bachelor of Science degree in Biological Sciences in May of 2013. He began his graduate studies in the laboratory of Dr. Alexander W.E. Franz in August of 2013, and completed his Doctorate of Philosophy in Veterinary Pathobiology from the University of Missouri in December 2018. 